\title{
The Experience of Family Members in the Context of Mental Illness: \\ Caregiving Burden, Personality Constructs and Subjective Well-being
}

\author{
Ella Amir
}

\author{
A Thesis \\ in
}

The Special Individualized Programs

Presented in Partial Fulfillment of the Requirements

For the Degree of Doctor of Philosophy at

Concordia University

Montreal, Quebec, Canada

November, 2011

(C) Ella Amir, 2011 


\section{CONCORDIA UNIVERSITY \\ SCHOOL OF GRADUATE STUDIES}

This is to certify that the thesis prepared

By: $\quad$ Ella Amir

Entitled: $\quad$ The Experience of Family Members in the Context of Mental Illness: Caregiving Burden, Personality Constructs and Subjective Well-Being

and submitted in partial fulfillment of the requirements for the degree of

DOCTOR OF PHILOSOPHY (Special Individualized Programs)

complies with the regulations of the University and meets the accepted standards with respect to originality and quality.

Signed by the final examining committee:

Chair

Dr. M. Mulrennan

External Examiner

Dr. M. Piat

External to Program

Dr. H. Petrakos

Examiner

Dr. D. Pushkar

Examiner

Dr. R. Reilly

Thesis Supervisor

Dr. C. Wrosch

Approved by

Dr. D. Howes, Graduate Program Director

January 24, 2012

Dr. G. Carr, Dean, School of Graduate Studies 


\begin{abstract}
The Experience of Family Members in the Context of Mental Illness:

Caregiving Burden, Personality Constructs and Subjective Well-being
\end{abstract}

Ella Amir, Ph.D.

Concordia University, 2011

This longitudinal study examined psychological factors that can contribute to subjective well-being among 147 individuals who are the primary caregivers of family members with mental illness. It was hypothesized that adaptive personality constructs and the personality profiles they create would predict higher levels of subjective well-being by facilitating coping with caregiving stress. In addition, it was investigated whether caregiving burden would mediate the associations between personality constructs, coping, and well-being, or whether personality would moderate the associations between burden, coping, and well-being.

The personality constructs included goal adjustment capacities (goal disengagement and goal reengagement, Wrosch, Scheier, Miller, Schulz, \& Carver, 2003), dispositional optimism (Scheier \& Carver, 1987), and unmitigated communion (Helgeson \& Fritz, 1998). Indicators of well-being included positive and negative affect, life satisfaction, depressive symptoms, and purpose in life.

The cross-sectional and longitudinal results indicated that goal disengagement, goal reengagement, and optimism predicted higher levels of several indicators of subjective well-being. Unmitigated communion was associated only with negative affect and depressive symptoms. Caregiver burden was negatively associated with goal 
disengagement and optimism, but positively with goal reengagement, and explained some of the effects of these personality constructs. Moreover, goal adjustment capacities predicted improved well-being among highly burden participants, while optimism was associated with higher well-being among their less burdened counterparts. In addition, some personality profiles, created by interactions between the three personality constructs, were meaningfully associated with subjective well-being. Finally, certain coping behaviors were associated with goal adjustment capacities and optimism but not with unmitigated communion. Effective and less useful care-specific coping mediated the effects of goal adjustment capacities and optimism on participants' subjective well-being.

Overall, the study's findings suggest that different personality constructs can influence the subjective well-being of individuals caring for mentally ill family members. Moreover, many of these effects could be statistically explained by the way individuals cope with caregiving stress. These findings have important implications for theories of personality functioning and adjustment to stressful life circumstances. In addition, they illuminate pathways to subjective well-being, which has important practical implications for helping caregivers manage their difficult life circumstances and improve their quality of life. 


\section{Acknowledgments}

Going back to school as a (very) mature student responsible for a communitybased organization that demands more than full-time attention, was not a light undertaking. Good alignment between my aspirations and the opportunity to fulfill them was imperative before a decision could be made. Concordia's Special Individualized Program provided the opportunity, but it was Carsten Wrosch, my supervisor, who is the principal reason this adventure could be accomplished. My heartfelt gratitude goes to Carsten, who was my guide throughout this journey, and who provided advice, support, and encouragement in myriad ways and then some. This 'expedition' could not have happened without him!

I thank Dolores Pushkar and Rosemary Reilly, who along with Carsten served as my committee, and who offered valuable input along the way. Thank you to Joelle Jobin, Fatima Amari and Robin Grumet for their assistance with data collection and other tasks.

Special thanks are owed to the members of AMI-Quebec, who are the primary reason for my interest in this research. While in part driven by scientific curiosity, it is the opportunity to influence the caregiving experience through improved programs and support that was my main motivation. In particular I thank the individuals who participated in my research and who provided a rich pool of information that can continue to be unearthed in the coming months.

Last but not least, I thank Shimmy, who believed in me in the moments when I was losing my confidence, and who was instrumental in helping me to reach the finish line. 
List of Appendices........................................................... viii

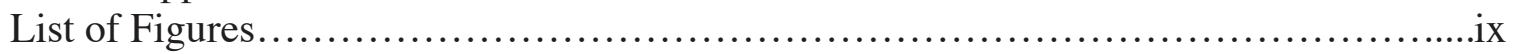

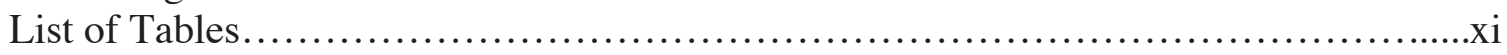

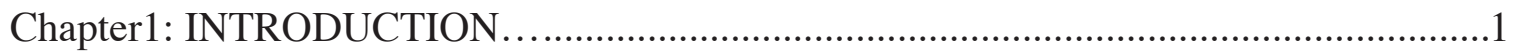

Caregiving and Burden.................................................... 3

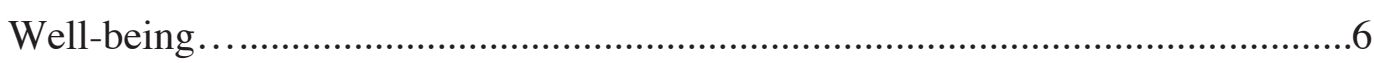

What is well-being?...............................................................6

Burden and subjective well-being in mental illness....................15

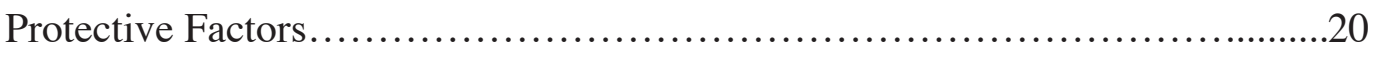

What is personality and the role of personality constructs ...............20

Personality and coping types.........................................24

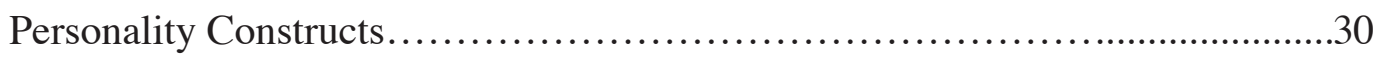

Goal adjustment capacities.........................................30

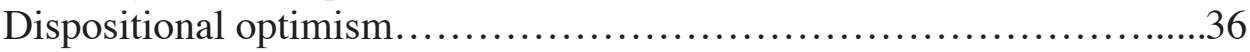

Unmitigated communion.....................................41

Chapter 2: THE RESEARCH........................................... 47

Theoretical Model........................................................47

The Present Research................................................48

Hypotheses....................................................... 51

Method................................................................. 52

Participants and procedures........................................52

Materials...................................................54

Chapter 3: RESULTS ...................................................60

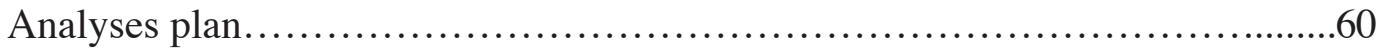

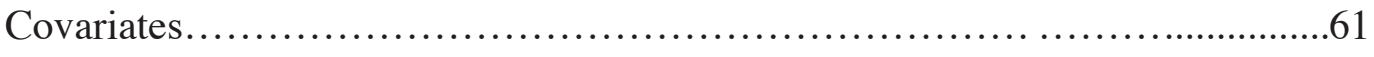

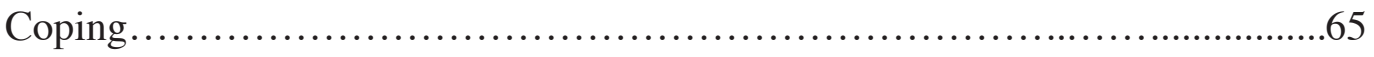

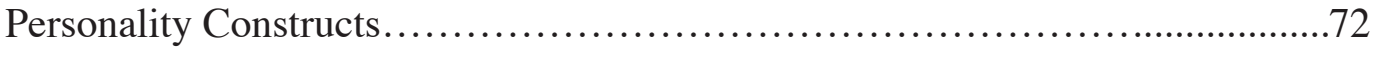

Correlations between constructs..................................72

Goal adjustment capacities.......................................................... 74

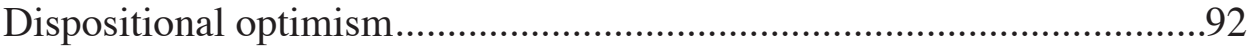




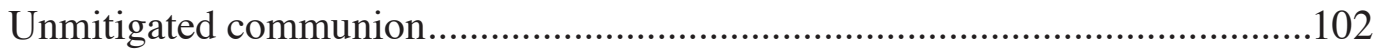

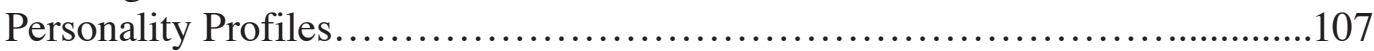

Chapter 4: DISCUSSION ............................................... 126

Goal Adjustment Capacities......................................................127

Dispositional Optimism.................................................. 131

Unmitigated Communion............................................. 135

Personality Profiles.........................................................

Contributions for Theory ................................................. 141

Implication for Practice............................................... 145

Limitations and Future Research..........................................150

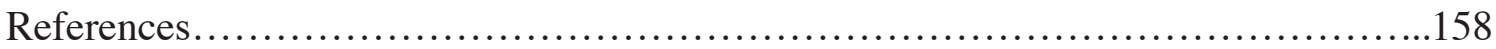

Appendices........................................................... 181 


\section{List of Appendices}

A. Pathways to Health and Well-Being Among Families living with Mental Illness Questionnaire

B. Consent form 


\section{List of Figures}

Figure 1

Associations between baseline levels of caregiver burden and depressive symptoms, separately for participants with high versus low baseline levels of goal reengagement capacities

Figure 2

Associations between baseline levels of caregiver burden and satisfaction with life, separately for participants with high versus low baseline levels of goal disengagement capacities

Figure 3

Associations between baseline levels of caregiver burden and changes in depressive symptoms, separately for participants with high versus low baseline levels of goal disengagement capacities

Figure 4

Associations between baseline levels of caregiver burden and changes in negative affect, separately for participants with high versus low baseline levels of goal disengagement capacities

Figure 5

Associations between baseline levels of caregiver burden and changes in satisfaction with life, separately for participants with high versus low optimism

Figure 6

Associations between baseline levels of caregiver burden and change in positive affect, separately for participants with high versus low optimism

Figure 7

Associations between baseline levels of goal disengagement and depressive symptoms, separately for participants with high versus low optimism 
Figure 8

Associations between baseline levels of goal disengagement and negative affect, separately for participants with high versus low goal reengagement

Figure 9

Associations between baseline levels of goal disengagement and change in purpose in life, separately for participants with high versus low optimism

Figure 10

Associations between baseline levels of goal disengagement and change in purpose in life, separately for participants with high versus low unmitigated communion

Figure 11

Associations between baseline levels of goal reengagement and change in negative affect, separately for participants with high versus low unmitigated communion

Figure 12

Associations between baseline levels of goal disengagement and self-blame separately for participants with high versus low goal reengagement capacities 


\section{List of Tables}

Table 1

Correlations between covariates and baseline indicators of subjective well-being

Table 2

Correlations between covariates and changes in indicators of subjective well-being (T2) controlled for baseline well-being (T1)

Table 3

Correlations between goal adjustment capacities, optimism, unmitigated communion and coping with caregiving stress

Table 4

Correlations between baseline levels of coping with caregiving stress and baseline (T1) and follow-up (T2) levels of indicators of subjective well-being

Table 5

Correlations between personality constructs (goal disengagement, goal reengagement, optimism and unmitigated communion)

Table 6

Cross-sectional results from hierarchical regression analyses predicting baseline levels of depressive symptoms, purpose in life, satisfaction with life, positive and negative affect, and caregiver burden, by participants' goal adjustment capacities (Model 1) and by the interactions between caregiver burden and goal adjustment capacities (Model 2)

\section{Table 7}

Longitudinal results from hierarchical regression analyses predicting baseline levels of depressive symptoms, purpose in life, satisfaction with life, positive and negative affect, and caregiver burden, by participants' goal adjustment capacities (Model 1) and by the interactions between caregiver burden and goal adjustment capacities (Model 2)

Table 8

Cross-sectional results from hierarchical regression analyses predicting baseline levels of depressive symptoms, purpose in life, satisfaction with life, positive and negative affect, 
and caregiver burden, by participants' optimism (Model 1) and by the interactions between caregiver burden and optimism (Model 2)

Table 9

Longitudinal results from hierarchical regression analyses predicting baseline levels of depressive symptoms, purpose in life, satisfaction with life, positive and negative affect, and caregiver burden, by participants' optimism (Model 1) and by the interactions between caregiver burden and optimism (Model 2)

Table 10

Cross-sectional results from hierarchical regression analyses predicting baseline levels of depressive symptoms, purpose in life, satisfaction with life, positive and negative affect, and caregiver burden, by participants' unmitigated communion (Model 1) and by the interactions between caregiver burden and unmitigated communion (Model 2)

Table 11

Longitudinal results from hierarchical regression analyses predicting baseline levels of depressive symptoms, purpose in life, satisfaction with life, positive and negative affect, and caregiver burden, by participants' unmitigated communion (Model 1) and by the interactions between caregiver burden and unmitigated communion (Model 2)

Table 12

Cross-sectional results from hierarchical regression analyses predicting baseline levels of indicators of subjective well-being by participants' personality constructs and their interactions

Table 13

Longitudinal results from hierarchical regression analyses predicting baseline levels of indicators of subjective well-being by participants' personality constructs and their interactions 


\section{Chapter 1}

\section{INTRODUCTION}

Caring for a mentally ill relative is a long-term responsibility that may subject the caregiver to significant burden. When extended over a long period of time, such burden can compromise the caregiver's own well-being, both physically and emotionally (Ory, Hoffman, Yee, Tennstedt \& Schulz, 1999; Schulz, O'Brien, Bookwala, \& Fleissner, 1995).

Caregiving is increasingly being recognized as an important experience affecting both care-recipient and caregiver, as well as having significant economic and social ramifications (e.g., Clark \& Drake, 1994; Clark et al., 1994; Grunfeld et al., 2004). While family caregiving is not a new concept, it has taken new urgency in recent times due to changing infrastructures of families and communities. With smaller and more dispersed families, a growing number of women in the work force, aging population and increased life expectancy, caregiving is becoming a reality for an increasing proportion of people. When, rather than if one is likely to become a caregiver, may well become the appropriate question in coming years.

The increased interest in caregiving, however, has been focused mainly on caregiving to frail elderly, especially to Alzheimer's patients and those with similar conditions (e.g., Chappell \& Reid, 2002; Gallant \& Connell, 1998). Caregiving in mental illness is under-represented in the caregiving research despite evidence of the important consequences associated with caregiving for a loved one with mental illness.

As noted, there are three principal players in the context of caregiving for individuals with mental illness: care-recipients, unpaid caregivers and health care 
providers. The effects of mental illness can present far-reaching challenges for the entire family, including care recipients and caregivers, as well as for care providers and the healthcare system at large. The adverse consequences for public health cannot be underestimated (Roth, Perkins, Wadley, Temple, \& Haley, 2009; Talley, \& Crews, 2007). In addition, the different individuals involved in the caregiving process can influence each other and thereby affect caregiving process and outcome (Ayres, 2000). While this implies that an optimal approach to studying caregiving would involve gathering information from different sources (i.e., caregivers, care recipients, and care providers), this thesis focuses on the experience of the primary caregivers, with special attention to the impact of mental illness on their subjective well-being. However, it also takes into account relevant characteristics of the care recipient (e.g., severity of mental illness), as perceived by the caregiver, and examines how caregivers cope with their mentally ill family members in stressful caregiving situations. This approach is likely to be fruitful as it may already shed some light on important interactions between caregivers and their mentally ill family members that can protect the family by helping to maintain a caregiver's subjective well-being. However, it should be followed up in future work by comprehensive assessments of all family members and care providers involved to substantiate conclusions drawn from this study.

The thesis explores the impact of caregiver burden on a sample of family members caring for a relative diagnosed with mental illness. Their subjective well-being was analyzed, and an attempt was made to understand how personality constructs, along with coping behaviors, may influence this outcome. 
The importance of such research extends beyond the mere understanding of the consequences of caregiving. Better understanding is expected to inform and guide policy recommendations and ultimately be instrumental in better supporting caregivers. Adequate support for caregivers is likely to translate into several benefits. First, caregivers may be better able to sustain their engagement as caregivers, thus benefiting their ill relatives. Second, proper supports can mitigate the potential negative consequences of caregiving to the caregivers' own well-being. Lastly, it is expected that healthier behaviors, of both caregivers and their ill relatives, would translate into higher recovery rates and reduced relapse rates and hospitalizations, and thus should reduce long-term mental health-related costs.

\section{Caregiving and Burden}

The burden associated with the role of family members as caregivers to a loved one with mental illness has been widely documented in the literature and reflects the overall level of distress associated with this role. Wasow (1995) observes: "As with a large stone skipping across water, the ripple effect of mental illness on the entire family is enormous" (p. 3). A typical trajectory of mental illness includes cycles of crises and remissions, often over many years, subjecting family members to ongoing burden and stress that can become chronic.

Gravitz $(2000,2004)$ suggests that chronic stress is the foundation of the family experience of mental illness. This is compounded by chronic trauma, which lies at the core of the family experience. "Stress stretches the fabric of the family; trauma tears it apart” (2004, p. 47). Additionally, families typically experience chronic loss and grief that reflect both concrete losses (such as economic burden, social and personal losses), 
and losses to privacy, freedom, security and even dignity. With a 'steady diet of loss,' Gravitz observes, families often go through protracted grieving that is often compounded by the lack of acknowledgment or legitimacy of grieving in mental illness. The result is chronic exhaustion that can lead to 'compassion fatigue', and to what Gravitz identifies as 'counterpart disorder.' Denial, minimization, high tolerance for inappropriate behavior, as well as confusion, doubt, guilt, depression, and low self esteem are typical to such 'disorder.' Physical and emotional symptoms, as well as demoralization are common.

Viewing families as causal agents that precipitate or exacerbate mental illness in a loved one through unhealthy communication (Mishler \& Waxler, 1965) or charged emotional environment (Vaughn \& Leff, 1976) was common a few decades ago. This perception is gradually changing, and has been replaced with a growing recognition that families are co-victims of what is widely viewed as a biologically-based illness that affects their relatives (Hatfield \& Lefley, 1987). However, while the blame is gradually lifting, families continue to be entrusted with the role of caring for a loved one with mental illness, a role often lasting a lifetime and carrying a significant level of burden.

Family members are often involved with providing care and support, regardless of the illness severity. However, families with relatives suffering from a severe mental illness (SMI) are most likely to assume long-term, often life-long, caregiving responsibilities. This accounts for an estimated 1.8 million families in Canada alone.

While there is no internationally accepted definition of severe mental illness (Ruggeri, Leese, Thornicroft, Bisoffi, \& Tansella, 2000) it is widely agreed that $5.4 \%$ of the population can be viewed as having a severe and persistent mental illness. The 
National Institute of Mental Health defines SMI as a diagnosis of non-organic psychosis or personality disorder involving prolonged (two years or more) illness and long-term treatment, including disability that meets at least three of eight specific criteria (Manderscheid \& Sonnenschein, 1996).

Lefley (1987) suggests that more than one third of adults diagnosed with mental illness live with their families, but some studies indicate higher proportions. Swan and Lavitt (1986) estimate the rate at 42\%, and Hatfield (1983) maintains it can reach 44\%. Tessler and Goldman (1982) observe that of the mentally ill who were not living in their family home, $90 \%$ were in contact with their families if they lived nearby. Talbott (1983) suggests that regardless of their living situation, $70 \%$ of adults with severe mental illness have family members who can or will be involved in their care.

Reinhard and Horwitz (1995) observe that the living situation of adults with mental illness is not necessarily indicative of the caregiver's burden. Many family members whose loved ones live on their own (or in supported settings outside of the family home) provide assistance that is associated with high levels of burden, therefore separate living arrangements do not necessarily imply less caregiving burden.

In-spite of the undisputed impact of mental illness on the entire family, there is not yet a common perception that mental illness is a 'family affair' (Family Caregiving Alliance, 2007). While family members usually wish to be involved in the care of their loved ones and appreciate an opportunity to be informed and referred to support programs that could assist them in their role, the healthcare system is commonly poorly equipped to address caregivers' needs (Family Caregiving Alliance, 2007). Many careproviders do not regard caregivers as their clients and feel ill-equipped to address their 
inquiries and needs. Moreover, care-providers are concerned about interference with the flow of the clinical process and their effort to establish trusting rapport with the patient (Family Caregiver Alliance, 2007).

Twenty-six percent of all Canadians reported having provided care for a family member or close friend during a 12-month period (Statistics Canada, 2002). Twenty-two percent of these caregivers missed one or more months of work and $41 \%$ used personal savings to fulfill their role. The intensity and length of unpaid work was significant, with more than $60 \%$ providing care for more than three years. Moreover, unpaid caregivers provided $80 \%$ of care needed by individuals with long-term conditions, and the economic value of caregivers' unpaid care to the Canadian economy was estimated at \$6-9 billion (Health Canada, 2002).

The long-term nature of mental illness and the stressors inherent in the caregiving situation, as described above, subject caregivers to significant levels of chronic stress. The unpredictability of the illness and the need to be persistent and vigilant present ongoing challenges to caregivers and compromise their own health (Schulz \& Beach 1999). Most caregivers are women (59\%-75\%; National Alliance for Caregiving, 1997), and the hardship they experience is reflected in a significant economic burden, demands on their time and important consequences to their physical and emotional health.

\section{Well-Being}

What is well-being?

As a long-term responsibility which is associated with burden and can be qualified as a chronic stressor, caregiving in mental illness can compromise a person's subjective well-being (Ory et al., 1999; Schulz et al., 1995). A complex construct, well- 
being is associated with physical, as well as with emotional health. Perceived by some researchers as the opposite, or lack of, ill-being, well-being is viewed by other researchers as a construct independent of ill-being (Ryff et al., 2006). It is associated with quality of life as well as with personality traits, and is influenced by both stable dispositions and situational emotions (Schmutte \& Ryff, 1997). The following section reflects some of the views about this complex construct.

For close to four decades the research on psychological well-being (PWB) was guided by three primary concepts of functioning: the distinction between positive and negative affect (Bradburn, 1969; Watson, Clark, \& Tellegen, 1988); and the emphasis on life satisfaction, a cognitive component, as a key indicator of well-being (Ryff, 1995).

Positive affect and negative affect have emerged in research as distinctive dimensions, largely independent of each other (Watson et al., 1988). Positive affect refers to a sense of enthusiasm and alertness, with high positive affect reflecting high energy, concentration and engagement, and low positive affect being characterized by sadness and lethargy (Watson et al., 1988). Negative affect is a general state of distress that includes moods such as anger, guilt, fear, and nervousness. Low negative affect reflects a state of calmness.

While positive and negative affect represent dimensions of affective states, studies have demonstrated that they are related to corresponding affective trait dimensions of positive and negative emotionality (Tellegen, 1985; Watson \& Clark, 1984). Moreover, trait negative and positive affect were linked to psychobiological and psychodynamic constructs of sensitivity to signals of reward and punishment (Tellegen, 
1985). Tellegen also suggests that low positive affect and high negative affect - both state and trait - are major variables that distinguish depression from anxiety.

While positive and negative affects refer to the emotional, affective aspects of PWB, satisfaction with life refers to a cognitive-judgmental process (Diener, Emmons, Larsen, \& Griffin, 1985). As such, it is a subjective measure based on people's own assessments of life, as compared with their own standards and not with any externally imposed criteria. Individuals may place different values on different dimensions (e.g., health, energy), therefore it is important to evaluate overall satisfaction rather than sum up specific dimensions (Diener et al., 1985).

Lucas, Diener and Suh (1996) note that the term 'subjective well-being' (SWB) refers to people's evaluation of their lives both cognitively and emotionally, and agree that while these dimensions are related, they are empirically distinguished from each other and need to be studied individually in order to comprehend the overall expression of subjective well-being.

Early attempts to measure satisfaction with life have often used scales that consisted of a single item only (Diener, Emmons, Larsen \& Griffin, 1985). Additionally these scales have been designed for certain populations and are not appropriate for others. For example, Neugarten, Havighurst and Tobin's (1961) Life Satisfaction Index, and Lawton's (1975) Philadelphia Geriatric Center Morale Scale. Moreover, many of the scales appear to measure more than life satisfaction, as they do not tap solely the judgmental quality of satisfaction with life.

Depression (or depressive symptoms), which is reflected through emotional expression, behaviors, physical and mental health, can have a significant impact on a 
person's overall well-being. In his seminal work on depression, Beck (1967) suggested that depression is reflected in the idiosyncratic way people view themselves, the world, and their relations with their environment. Depressed people perceive their lives as filled with burden and obstacles and interpret the outcomes of their actions as failure and defeat. Additionally they view themselves as inadequate and deficient and have little hope for a better future. People who are exposed to the continuous burden of caregiving are at risk of developing depressive symptoms and compromising their general wellbeing (Beck, 1967). For example, Carter and Chang (2000) found that a majority of caregivers to cancer patients who experienced depressive symptoms at a level that would suggest a risk for clinical depression, also experienced severe sleep disturbances. Depression, therefore, can be viewed as an outcome of the caregiving experience, as well as an influencing factor on the general well-being of the caregiver.

In addition to the experience of emotions, depression, and perceptions of life satisfaction, psychological well-being has been proposed to include other dimensions, such as environmental mastery and purpose in life (Ryff, 1995; Ryff et al., 2006). These dimensions have not been addressed by the measurement of emotions and life satisfaction.

Another concept related to well-being is quality of life. Like well-being, the understanding of what quality of life means varies from one researcher to another. Borthwick-Duffy (1992) distinguishes between objective indicators (such as life conditions) and subjective indicators (such as satisfaction with life). Some researchers use a single item to assess quality of life (Andrews \& Whitey, 1976), while others use multiple items related to various life domains (Cummins, 1996). Additionally, the 
person's appraisal of a given life domain as more or less important, is also associated with individuals' perception of their quality of his life.

Other constructs viewed as related to quality of life are goal adjustment capacities and optimism. Carver and Scheier (1981) observe that the importance of goals in relation to quality of life stems from the role of goals in providing a general framework that instills purpose and direction in one's life.

The relation between well-being and personality presents yet another potential issue. Schmutte and Ryff (1997) suggest that psychological well-being is distinct from personality and affect and falls between the two. They observe that while personality represents distinct and stable constructs that affect behaviors and thoughts, well-being is subjected to change through the life span and reflects developmental accomplishments, life events and context. They attribute the ambiguous distinction between the two, in part, to the tendency of previous studies to operationalize well-being mainly in terms of affect. In response, they refer to the multi-dimensional model of psychological well-being (PWB) that was conceptualized by Ryff (1989a, 1989b, 1995), and attempted to preserve the conceptual distinction between personality and well-being while broadening the definition of well-being to include additional facets of psychological functioning.

Ryff's model (1995) includes six distinct components of positive functioning: self-acceptance, personal growth, purpose in life, positive relations with others, environmental mastery, and autonomy. In a 1989 study Ryff focused on age and gender profiles to better understand the associations of these variables with well-being, and found incremental age profiles for environmental mastery and autonomy; decremental age profiles for purpose in life and personal growth; and no age differences for self 
acceptance and positive relations with others. In this study and others that followed (Ryff \& Keyes, 1995; Ryff, Lee, \& Na, 1993), women scored significantly higher than men on positive relations with others and on personal growth.

Ryff's model to investigate well-being represents a significant improvement in comparison with other alternatives, especially the single-factor models. Moreover, mixed relationships between Ryff's model components and previously used variables, suggest that relying on earlier variables alone would lead to neglecting other important aspects of well-being such as the ones she proposes.

In an attempt to measure well-being, Schmutte and Ryff (1997) asked participants to judge their life outcomes, instead of reporting on the frequency of positive and negative feelings. Like personality, these reports cover a longer period of time of the typically measured "recent history". Unlike personality, well-being measures reflect participants' perception of the quality of their lives, rather than their personality traits. Additionally, while previous inquiries view well-being as being influenced significantly by adulthood life events, Schmutte and Ryff (1997) observe that personality is traditionally viewed as an antecedent of life events. They suggest, therefore, that these dimensions of psychological well-being are both distinct from, but significantly influenced by personality.

Growing interest in positive psychology in recent years has triggered the question whether well-being and ill-being represent separate, independent dimensions of mental functioning, or whether well-being is just the flip-side of psychological maladjustment (Ryff et al., 2006). The latter views well-being and ill-being as opposite ends of one continuum, suggesting that what has been learned about ill-being can be transferred to 
well-being. For example, a high score on depression would be expected to correspond to a low score on happiness or purpose in life, and vice versa. In contrast, the independence view regards well-being and ill-being as two distinct, but related, properties and suggests that extrapolation from one to another (i.e., causes, consequences) may be misleading (Ryff et al., 2006). In support of the independence paradigm, studies have shown that the absence of ill-being (e.g., depression) is not a guarantee of high well-being (Keyes, 2002; Singer, Ryff, Carr, \& Magee, 1998). Moreover some individuals have shown to be high on both well-being and ill-being scales, while others showed no psychological disorders but lack meaningful life engagement at the same time.

Ryff and her colleagues (2006) examined the empirical associations between psychological well-being and ill-being with various biomarkers, both neuroendocrine and cardiovascular. While the study design did not include the causal directionality (does well-being or ill-being influence biology; does biology influence both; or are they reciprocally related), the results are important in that they examined the links between positive and negative well-being measurements with biology in the same study, which has been seldom done before, if at all. The overall findings were supportive of the distinct hypothesis. More specifically, for seven biomarkers, significant correlations with wellbeing (or ill-being) were not accompanied by significant correlations with ill-being (or well-being, respectively) and the same biomarker. Moreover, in most of the correlations that reflected the distinct pattern (69\%), measures of well-being were significantly correlated with biomarkers but no similar effects were found for measures of ill-being. Overall, psychological well-being, in contrast to psychological ill-being, showed a stronger association with biological markers. Additionally, higher well-being was 
associated with lower biological risk, and conversely higher ill-being was associated with higher biological risk. Some anomalies (such as a significant association between higher levels of negative affect, anxiety and anger with lower levels of systolic blood pressure) coupled with the study's small sample size, suggest caution in interpreting the findings, however they add to the growing literature on the associations and influences between well-being and biology (Ryff et al, 2006).

Caregivers, as compared with non-caregivers, have shown significant differences on biological markers of functioning (Kiecolt-Glaser et al., 2003). This can be further explored by studying psychological well-being and ill-being, especially since caregiving can be linked with both heightened distress and enhanced well-being under certain conditions, such as absence of work-family role conflict (Marks, 1998). Caregivers who find meaning, a strong sense of purpose and connectedness in their caregiving role, and an absence of conflicting role demands, may show different biomarkers than their counterparts who don't experience the same sense of well-being. Additionally, adaptive response to challenge, termed by Charney (2004) the phenomenon of resilience, suggests that resiliency is characterized by some of the same biomarkers studied by Ryff and her colleagues (2006).

Personality and well-being literature continues to be challenged by the need to define the association between stable dispositions and situational emotions. As mentioned earlier, the attempt to understand psychological functioning in terms of emotional states (i.e., affect and well-being) or in terms of emotional traits (i.e., personality), has not been as clear when the operationalization of personality and affect was attempted (Schmutte \& Ryff, 1997). These two distinct concepts refer to the 
difference between the experience of a current affect and the enduring tendencies to experience such affect (Schmutte \& Ryff, 1997). However, when happiness, for example, was examined, they observed that while happiness is a state, it might be influenced not only by external circumstances but also by internal dispositions. States and traits, it appears, "are so intimately tied that it is often difficult to distinguish..." (McCrae \& Costa, 1991, p. 227). The use of primarily self-reports in the investigation of personality and well-being, poses a validation challenge when other sources of data are not available, and thus further complicates this already complex conundrum.

Inglehart and his colleagues (2008) explored well-being and happiness of individuals and societies in a world that has gone through some dramatic changes in the last quarter century. They note that while similar, happiness and life satisfaction represent different aspects of subjective well-being. Happiness reflects the objective experiences of people, as well as how they evaluate these experiences against their values and beliefs. Life satisfaction is a measure more sensitive to economic conditions than happiness. For example, in many ex-communist countries where dramatic political and social changes were accompanied by economic breakdown, happiness increased, but life satisfaction decreased.

Inglehart and his colleagues' (2008) longitudinal study of 52 countries during the period 1981-2007 led to the suggestion that in low-income countries economic factors are closely linked with subjective well-being (SWB). In such societies happiness is associated with group solidarity, as well as with religiosity and national pride. However, when economic security increases, self-expression and free choice appear to be more important than solidarity. In other words, while "the transition from a society of scarcity 
to a society of security brings a dramatic increase in subjective well-being" (Inglehart, 1997, p. 64), at a certain threshold further economic gains do not necessarily increase SWB; non-economic factors then become more important for people's SWB, as well as for societies' SWB.

Fischer and Boer's (2011) study supports this observation and note that autonomy has an overall larger and more consistent effect on people's well-being than money: "Money leads to autonomy but it does not add to well-being or happiness" (p. 180). Mapping the structure of well-being, therefore, is a complex undertaking that requires ongoing study of multi-sourced evidence (Ryff, 1995). For the purpose of this study, the complexity of the well-being literature will be addressed by covering a wider range of constructs. In this regard, this dissertation will measure positive affect, negative affect, life satisfaction, depressive symptomatology, and purpose in life as indicators of subjective well-being.

\section{Burden and subjective well-being in mental illness}

Like well-being, burden is a multidimensional concept (Schene, Tessler, \& Gamache, 1994). In the context of caring for a mentally ill loved one, researchers have distinguished between objective burden and subjective burden. Objective burden refers to the actual hardship and disruptions associated with caregivers' duties; subjective burden reflects the personal suffering endured by the caregiver. Grief, chronic sorrow, an emotional roller coaster and empathic pain are central themes in describing the subjective burden of families (Marsh, 2001; Marsh et al., 1996). These experiences are difficult to quantify but their impact on the caregiver are dramatic, traumatic, and often lasting a lifetime. 
Objective burden is related to the practical problems associated with the illness. It includes dealing with positive (psychotic) and negative (deficit) symptoms, mood disturbances, harmful, disruptive or self-destructive behaviors, as well as socially or personally inappropriate manners (Marsh, 1998). Additionally, caregivers need to cope with new and unfamiliar requirements imposed by the illness. Accessing mental health services is often a challenge. A shortage of services is common in many communities and families often have to deal with unfriendly healthcare professionals, adding not only to the objective burden but also to their sense of helplessness and incompetence. This may prove especially challenging at the beginning of their journey, when families tend to be vulnerable and susceptible. This burden may be further compounded by the experience of negotiating with the legal and criminal justice systems (Lefley, 1996). Financial burden, disruption to employment, challenges to their marital and family relations, and interferences with their social life further add to the burden caregivers commonly experience. Families who endure such challenges for a long time are vulnerable to burnout and exhaustion.

While objective burden is associated with actual caregiving activities, families who are not involved with their relatives' practical care still suffer the impact of subjective burden. Circumstances may require separation from an ill relative (because of conflict or repeated crises), however this separation does not free caregivers from the impact of subjective burden. In fact, it may exacerbate a sense of guilt, helplessness and loss.

Reinhard and Horwitz (1995) observe that most parents report at least some negative consequences of caregiving. Objective burden is most frequently associated with 
being distracted from activities and household routines, as well as with family frictions. Worrying about the future is the most cited component of subjective burden; grieving a loved one's 'old self' and concerns about effective communication were commonly cited as well (Reinhard \& Horwitz, 1995). Siblings report on objective burden similarly to parents', however in the subjective domain, almost all siblings cited worries about the future and their role as caregivers as their parents age. Siblings' grief was similar to parents' and stigma ranked higher as subjective burden compared with parents (Reinhard \& Horwitz, 1995)

Lefley (1987) pays special attention to the consequences of burden on aging family caregivers. She observes that for aging parents, "lifetime caregiving may be an excessive burden with the potential for grave risks to health, both psychological and physical" (p. 1068). Instead of providing a support and additional resource to the mental health system, many caregivers provide day-to-day care and serve as case managers for their loved ones, responsibilities that often exact high price on their own well-being. "Caregiving for one group at the expense of another can scarcely be considered a desirable mental health objective" (Lefley, 1987, p. 1069).

An important component of burden in the context of mental illness is the stigma associated with mental illness. Kirby and Keon (2006) highlight the damaging effect of stigma on people diagnosed with mental illness and their families. Prevalent in society and often reflected in the interactions with service providers, stigma is commonly considered the most incapacitating handicap associated with mental illness, and exacerbate an already significant burden experienced by caregivers. 
Rosenheck and colleagues (2000) suggest that burden is related to the caregiver's appraisal of the situation. Experience of stress, therefore, is related to the appraisal of events (Lazarus, 1966, 1981): Only events viewed as threatening or harmful will lead to stress appraisal. Events which are considered irrelevant, benign or positive, do not lead to a stress reaction. Thus people first perceive events, then they determine how they deal with these events. Difficult symptoms in a relative may be, therefore, more or less burdensome, given the caregiver's perception, as well as the availability of resources for the patient. For example, difficult symptoms can be mitigated by the reduction of time the caregiver spends with the ill relative (Rosenheck et al., 2000). It is, therefore, important to distinguish between the content of burden and the consequences of the burdening activities. To understand the subjective experience of caregiving in mental illness it is important to disentangle the two and assess their impact on the caregiver's experience. While caring for adult children is generally not perceived a normative task, those who perceive caregiving as a normatively expected behavior (e.g., minorities, women, parents) are likely to experience less burden from the same level of caregiving responsibilities than those who do not consider caregiving a normative role (e.g., whites, men, siblings) (Reinhard \& Horwitz, 1995).

A literature review on caregiver burden in mental illness (Baronet, 1999), revealed associations between the burden of caring for a family member with mental illness and various variables, including sociodemographics, illness-related, and caregiver -related variables. For example, burden was associated with caregivers' age, ethnicity and residing with the ill relative, but not with the caregiver's gender, education and income, or the kinship with the ill relatives and their gender. 
,Joint residence with the ill relative was associated with increased objective burden (OB) but not with worry (subjective burden, SB), suggesting that caregivers worry about their ill relative even if they don't reside in the same household (Baronet, 1999). In various studies symptomatic behaviors were found to be associated with various variables such as objective burden, worry, stigma, fears, overall subjective burden, and overall burden, nevertheless symptomatic behavior was found to be the strongest and most consistent variable associated with burden in general.

Among caregiver stress and psychological resources variables, Baronet (1999) gleaned from the reviewed studies that when caregivers received support for dealing with their ill relative's illness (professional or through self-help groups), OB was reduced. Subjective burden, however, as well as overall burden were not affected by such supports. On the other hand social sources of support were shown in other studies to have mixed associations with SB or overall burden and were not associated with OB.

Baronet's (1999) overall conclusions of her literature review indicate that OB was associated with symptomatic behaviors and residing with the ill relative, and negatively associated with professional support. SB was positively associated with symptomatic behaviors and negatively with sense of mastery. Overall burden was positively associated with ethnicity (being white), symptomatic behaviors and amount of care provided.

Worry, stigma and fears (all SBs) were positively associated with symptomatic behaviors. As well, worry was positively associated with amount of care provided; stigma was negatively associated with caregivers' age, and fears were negatively associated with caregivers' age, education, and income or social class (white only). 
Treating burden as a mediator of various outcome variables in the reviewed studies revealed a positive association between objective, subjective, and overall burden, and psychological distress. One study found a positive association between subjective burden and depressive symptomatology (Song, Biegel, \& Milligan, 1997); another found a negative association between subjective burden and caregivers' positive assessment of their relations with their ill relative (Pickett, Cook, Cohler, \& Solomon, 1997); yet another found no association between subjective burden and adaptive coping (Solomon \& Draine, 1995). These findings underline the impact caregiver burden has on the caregiver's well-being and life in general (Baronet, 1999). Other studies reinforced the association between burden and compromised levels of subjective well-being (Ory et al., 1999; Schulz et al., 1995), and observed that this can be pronounced among individuals who experience high levels of care-related strain (Grunfeld et al., 2004; Schulz et al., 1997).

Another explanation for the chronic stress caregivers experience is that caring for a family member with mental illness often involves placing personal needs and goals after those of the relative (Baronet, 1999), a phenomenon that can render desired goals unattainable (Wrosch, Amir, \& Miller, 2011).

\section{Protective Factors}

\section{What is personality and the role of personality constructs}

Early personality researchers explained personality in terms of traits. Traits have been understood as typical behaviors stemming from genetic factors. McClelland (1951) noted that "trait psychology represents one of the earliest attempts to introduce some kind of order into the multiplicity of human responses. Its approach is simple. It consists of 
looking for consistencies in behavior" (p. 117). This approach also regards personality as dispositions, recognizing the influences people's psychological tendencies have on their reactions to the environment. Allport (1955) noted that "the most comprehensive units in personality are broad intentional dispositions, future pointed.... unique for each person, and tend to attract, guide, inhibit, the more elementary units to accord with the major intentions themselves..." (p. 92).

However, the continuous relationship between individuals and the environment in which they live cannot be under-estimated as an important influence on the evolution of one's unique characteristics. Hollander (1967) noted: "the individual personality is both influenced by and influences social processes" (p. 273). Murphy (1947) suggested that personality can be understood as arising from a 'bio-social' process, and recognized that neither biological nor social influences can be observed in isolation. Linton (1945) observed that an individual and his environment are interrelated in such a constant and dynamic interaction, that it is difficult to draw lines of demarcation. For that reason, he suggested that the definition of personality is one of delimitation.

Allport (1955) stated that the individual exerts influence on his environment in a pro-active way through a range of responses. In that way, "personality is less a finished product than a transitive process. While it has some stable features, it is at the same time continually going under change" (p. 19). Personality, thus, is an "open system" in which transactions take place between the person and the environment (Allport, 1960), and where the individual is the focus of social transaction processes.

Despite calls by various personality researchers in the 1960s to revise personality theories and to look for consistency in people's behaviors (through their interactions with 
each other and in interaction with the environment) as the main reflection of individual differences (Helson, 1964; Hunt, 1965; Miller \& Hamblin, 1963), Allport (1966) continued to argue that the variance in behavior is rooted in "idiosyncratic attitudes and traits" (p. 2), rather than in the situation. He observed that especially sociologists and anthropologists had used explanations in terms of "outside structure" rather than the "inside structure" (Allport, 1955). The fact that variables such as sex, age or social status influence one's outlook on life does not change the fact that "the outlook is a functioning part" of the individual. "Demography deals with distal forces - personality studies with proximal forces" (Allport, 1966, p. 2). Tendencies, capacities, and dispositions are internal to the person, "lie within", and they have the capacity to guide their behavior, “specific phasic reactions" (Allport, 1966, p. 2). By using the term traits, he included long-range sets and attitudes, as well as such variables as "“perceptual response dispositions', 'personal constructs' and 'cognitive styles"' (p. 3). Allport was cognizant of the complexity of the term. Like other intervening variables, traits are inferred rather than directly observed, thus discovering their nature presents a challenge. Yet, and despite his view of personality as an 'open system' as mentioned earlier, Allport insisted that traits cannot be explained in terms of interactions because they derive their energy from within the person. Notwithstanding he acknowledged that the great variability of behavior cannot be overlooked.

In somewhat different vein, personality can be seen as having external and internal levels, referring to its overt manifestations, and to its inner expressions. Additionally, it has a dynamic aspect - reflecting an ongoing change - and a consistent aspect, which provides continuity over time (Hollander, 1967). While the external level 
reveals some qualities (of the personality), the full spectrum of the personality cannot be grasped without the intra-psychic intricacies that include the individual's values, interests, motives and attitudes. The dynamic aspect of personality reflects the ongoing learning and experiences one acquires, which allow for change to happen. On the other hand there is a certain way in which individuals approach the world, cope with frustration or set up goals for themselves. Personality, Hollander (1967) concluded, "involves stable cognitive processes that generate a characteristic "style"' (p. 277).

Many researchers view personality as a way people think, behave, and experience the world around them, in a way that provides continuity, stability and consistency over times and situations (Carver, Scheier \& Segerstrom, 2010). Additionally, personality communicates certain uniqueness, or identity, which distinguishes one individual from another. In that vein, personality can also be viewed as providing the internal 'generator' for one's goals and associated behaviors, thoughts or feelings, which goes beyond the explanation of an external situation (Carver \& Scheier, 1990).

Various models attempt to explain the structure of personality, central among them is the five-factor model. Developed by Costa and McCrae $(1997,1980)$, the model initially included neuroticism, extraversion, and openness to experience, but has been revised to include measures of agreeableness and conscientiousness (Costa \& McCrae 1985; Wiggins, 1996). Personality constructs not included in such models, however, are of interest and are of particular relevance to our study. For example, Wrosch and Scheier (2003) suggest that personality factors can be associated with both the ways people manage life situations and with the outcomes of these situations. In that respect they refer to goal-related tendencies, such as optimism and goal adjustment as two personality 
constructs that can influence the quality of one's life through the role they play in selfregulatory activities.

\section{Personality and coping types}

For the past three decades, much research interest has been focused on the ways people cope with stressful situations in their lives (Carver \& Scheier, 1994). Lazarus and his colleagues (e.g., Lazarus, 1966; Lazarus \& Folkman, 1984) were pioneers in this research, and suggested that stress is experienced through two appraisal processes, along with the coping responses generated by it. Appraisal is related to the actual threat (or challenge), as well as to the response to the threat. Coping strategies are often grouped into two major types: problem-focused and emotion-focused behaviors. Folkman and Lazarus expanded the definition in their Ways of Coping Inventory (1980) and included six main types of coping in responding to stressful situations: problem-focused coping, seeking support, focusing on the positive, distancing from the stressful situation, wishful thinking, and self-blame. While the theoretical distinction between problem-focused and emotion-focused behaviors appears to be straight forward, Carver and Scheier (1994) argue that they typically co-occur and are difficult to separate from each other. For example, emotion-focused coping can facilitate problem-focused coping by removing some of the distress that can prevent effective problem-focused coping; on the other hand problem-focused coping can facilitate emotion-focused coping by presenting a threat as less threatening thereby reducing distress emotion.

Coping can also play a role in the caregiving experience. For example, Stengard (2002) identified different types of caregivers for relatives diagnosed with mental illness and explored their associations with coping strategies, need for support, and the 
caregivers perception of their own health and social disability, as well as that of their ill relatives. Five types were identified through a cluster analysis of four variables that included tension, worrying, supervision and urging. The emerging caregiver types included supervising, anxious, coping, resigned and activating caregivers. Supervising and anxious caregivers were found to be the most burdened, who also reported poorer physical and mental health, more psychological distress and more overload than other caregivers.

Supervision was associated with more severe symptoms in the care recipient, and found to be associated with emotional coping strategy (consistent with Reinhard \& Horwitz, 1995 findings). As well, supervising caregivers used problem solving, reappraisal and stress reduction more frequently than other types of caregivers. Stengard (2002) notes that problem-solving strategies are likely to be useful when the situation can be changed. When a difficult situation cannot be changed, problem-solving efforts may be not only ineffective but can further exacerbate the caregiver's stress. It appears, therefore, that the choice of coping strategies depends in part on the patient's symptoms and disability and in part on the caregiver's personality.

Traditionally, much attention has been given to dysfunctional, rather than positive coping in relation to stress. For example self-blame and wishful thinking (Bolger, 1990), escapism (Carver, Scheier, \& Weintraub, 1989; Rohde, Lewinsohn, Tilson, \& Seeley, 1990), all "avoidance" types of coping (Carver \& Scheier, 1994), have shown to be disadvantageous rather than helpful. Moreover, coping was found to be associated more with what is perceived as a threat than with what is perceived as a challenge (Carver \& Scheier, 1994). Threats were found to evoke a wide range of coping, both problem and 
emotion-focused. This is understandable, since unlike feelings of threat, feelings of challenge are positive and there is no need to reduce them when they arise (Carver \& Scheier, 1994). Considering that coping literature focused more on coping tactics that interfere with good outcomes than on coping tactics that facilitate good outcomes, led some to wonder whether coping does have positive effects (e.g., Aldwin \& Revenson, 1987).

Viewing coping as a process in which the person and the stressful event interact over time, and in which certain coping can be more or less useful at different points in time, poses a challenge when subjects are asked to envision stressful situations and describe their coping reactions to these situations (Carver \& Scheier, 1994). Since appraisal of the threat may be quite different from one situation to the next, controlling the characteristics of the stressful event is difficult. Carver and Scheier (1994) address this problem by choosing an event that is generally viewed as stressful, and explore the reactions to its different phases. A major exam (and the evaluative outcome attached to it), they suggest, is a situation that most people are exposed to at some time in their lives; it presents both a potential threat (negative outcomes) and a challenge (positive outcomes). Moreover, it is made up of several phases, including the preparation for the exam, the exam itself, the uncertainty about the results, and finally dealing with the actual outcomes. Folkman and Lazarus (1985) use the exam situation to explore coping behaviors in their study, which is probably the prototype of this research. They found that participants indeed differ in their coping and emotional reactions across the different phases of the situation. 
Another challenge in this context refers to the difference between situational coping and dispositional coping.

Lazarus' (1981) assertion that coping is a process and that coping behaviors may change from moment to moment in response to the stages of the stressful situation, raises the question whether one can speak of coping styles at all. Carver and Scheier (1994) suggest that people may develop certain ways of dealing with stress and that these patterns may be applied in new stressful events; in other words coping styles (dispositional coping) can influence situational coping (e.g., through "main effect", when a person reporting a tendency to use active coping, uses same coping at each phase of the stressful situation, regardless of its efficacy).

Cross-sectional and longitudinal studies suggest that effective coping (e.g., acceptance, positive reframing, active coping, or planning) can be associated with less burden and higher levels of emotional well-being among caregivers of mentally-ill relatives. By contrast, coping strategies such as self-blame, venting, denial, avoidance, or resignation, have been associated with higher levels of emotional problems (Dyck, Short, \& Vitaliano, 1999; Kim, Schulz, \& Carver, 2007; Magliano et al., 2000; Pratt, Schmall, Wright, \& Cleland, 1985; Rose, 1996; Seltzer, Greenberg, \& Krauss, 1995). As well, research has documented that the use of effective coping strategies (i.e., positive reinterpretation or planning) can buffer the adverse effect of high caregiving demands on elevated levels of depressive symptoms (Seltzer et al., 1995).

Bolger (1990) observes that people react to stress in different ways: while some remain resilient and their performance is not compromised by stress, others become distressed and their performance is affected by it. Coping researchers suggest that such 
outcomes are related to people's attempts to alter stressful situations and to regulate their emotional reactions through certain coping behaviors (Lazarus \& Folkman, 1984). Coping, Bolger (1990) states, is "a process explanation of differences in stress outcomes" (p. 525). Since personality constructs may also explain individual differences in reaction to stress, Bolger suggests that the two may work together in response to stressful events. To explore such a link between personality disposition and coping choices, he investigated whether coping processes mediated the effects of neuroticism (a trait similar to anxiety) on psychological distress and performance under stress (Bolger, 1990).

Recognizing that personality has an important role in influencing well-being outcomes, Bolger and Zuckerman (1995) suggest that personality can influence the exposure to stress, the reaction to stressful events, or both. Furthermore reactivity can be divided into coping choice and coping effectiveness and personality may be associated with either one of them or with both.

Exposure refers to the level of stress individuals experience, and reactivity refers to their emotional or physical reactions to a stressful experience. Bolger and Zuckerman (1995) present the different possible associations between personality and stress (exposure and reaction) but argue that an optimal model needs to consider the impact of personality on both stress exposure and stress reaction. They suggest that the association between personality and exposure may reflect a mediating effect on outcome, meaning that personality leads to exposure to stressors, and in turn leads to outcomes. The association between personality and reactivity, on the other hand, reflects a moderating process, which means that personality affects outcomes by moderating the effects of stressful events on these outcomes. In other words, this possibility (termed the differential 
exposure-reactivity model) suggests that personality affects both the exposure and reactivity to the stress experience in different ways (Bolger \& Zuckerman, 1995).

Bolger and Zuckerman (1995) note a similar association between personality and coping (choice and effectiveness). Coping choice refers to the behaviors people choose in the face of stressful events. Coping effectiveness is the extent to which the selected behaviors reduce the negative outcome associated with the stressful event. The differential choice-effectiveness model suggests that personality influences the choice of coping behaviors (mediation), as well as the coping effectiveness (moderation), and that both processes may explain personality differences in stress outcomes (Bolger \& Zuckerman, 1995).

To test their model, Bolger and Zuckerman (1995) explored these processes stressor exposure, stressor reactivity, coping choice and coping effectiveness - as possible explanations for the effect of neuroticism on distress in daily life, as reflected in anger, anxiety and depression outcome. They found that while reactivity was a more important process than exposure, high-neuroticism participants experienced exposure to more daily conflicts and were more likely to react to them with anger and depression. Additionally in the case of anger, neuroticism influenced coping choice but not coping effectiveness. High-neuroticism participants used more self-controlling and confronting coping, but these behaviors were found to be equally ineffective in both groups. Thus, in relation to anger, neuroticism triggered different coping choices that were translated into differences in anger, thus served to mediate the relationship between neuroticism (personality) and reactivity (to stress). Such mediational relationships were not found between neuroticism, self-controlling and escape-avoidance (as coping) and depression. 
Self-control was effective in preventing depression in low-neuroticism individuals, but it proved ineffective in high-neuroticism participants. Escape-avoidance was not related to depression in high-neuroticism participants, but did lead to an increase in depression in low-neuroticism individuals. The results, therefore, support a differential coping choiceeffectiveness model in regard to depression. In other words, to understand highneuroticism participants' depression as a reaction to conflict, it is important to consider both their coping choices and the effectiveness of these choices.

\section{Personality Constructs}

Assuming that caregiving in mental illness can be qualified as long-term burden that may trigger chronic stress, and recognizing that caregiver's personality and the coping behaviors associated with it can influence well-being, it is important to explore how caregivers' subjective well-being is influenced by their personality and the coping strategies they exercise. While it is recognized that care-recipient, as well as careprovider-related factors have an important impact on the caregiver's subjective wellbeing, this study has focused mainly on the caregiver's personality and associated coping behaviors. For the purpose of this study, three personality constructs were selected: goal

adjustment capacities (including goal disengagement and goal reengagement), dispositional optimism, and unmitigated communion. In the following sections these constructs and their potential associations with the caregiving experience are described.

\section{Goal Adjustment Capacities}

Theories of adaptive self-regulation postulate that personal goals structure people's lives, motivate adaptive behaviors, and contribute to high levels of subjective well-being (Carver \& Scheier, 1981; Carver \& Scheier, 1998; Emmons, 1986; 
Heckhausen, Wrosch, \& Schulz, 2010; King \& Hicks, 2007). A common view associates the determination to achieve goals with subjective well-being. At times, however, individuals confront challenges that render the attainment of desired goals impossible; in such situations the persistence to attain goals may be counter-indicative and may do the exact opposite, compromise their well-being (e.g., getting older, having to care for a sick child, or becoming unemployed) (Carver \& Scheier, 1998; Wrosch, Miller, Scheier, \& de Pontet, 2007; Wrosch \& Scheier, 2003). The outcome of persisting to realize a goal, therefore, needs to be viewed in a broader context: while under certain circumstances it is likely to affect well-being positively (e.g., higher levels of satisfaction, positive affect, purpose in life), in other situations it may compromise subjective well-being (e.g., increased depressive symptoms or negative affect). Additionally, despite the commonly viewed adverse consequences of goal failure, it has also been suggested that challenge and failure can provide opportunities for adaptive development (King \& Hicks, 2007) and individuals may thrive in problematic situations if they effectively regulate the experience of unattainable goals (Wrosch, Scheier, Miller, Schulz, \& Carver, 2003). Such adaptive effects in the context of goal failure are thought to depend on individual differences in two self-regulation capacities, which can influence whether goal failure compromises subjective well-being. These self-regulation capacities are associated with goal disengagement and entail the tendency to withdraw effort and commitment from an unattainable goal. In addition, they involve the tendency to reengage in alternative goals when unattainable goals are encountered, which incorporates the identification of, commitment to, and pursuit of alternative goals (Wrosch et al., 2007; Wrosch, Scheier, et al., 2003). 
In support of these ideas, cross-sectional and longitudinal research has demonstrated that goal disengagement capacities can predict reduced levels of depressive symptoms or negative affect, while goal reengagement capacities have been associated frequently with greater purpose in life and higher levels of positive emotions (Bauer, 2004; O'Connor \& Forgan, 2007; Wrosch et al., 2007; Wrosch, Miller, \& Schulz, 2009; Wrosch \& Scheier, 2003).

These effects have been explained by the primary function of goal disengagement capacities, which is to prevent accumulated failure and the associated emotional distress. Goal reengagement capacities, by contrast, are mainly directed at the pursuit of new purposeful goals, which is thought to increase positive aspects of subjective well-being (Wrosch et al., 2007). Some studies have shown deviations from this pattern of results, in that goal reengagement can be associated with lower levels of negative mood, and goal disengagement can predict positive indicators of subjective well-being (Wrosch et al., 2007, Wrosch et al., 2003). Such associations may occur when goal disengagement provides resources that facilitate the pursuit of other new goals, and goal reengagement reduces some of the negative emotions deriving from the continued pursuit of an unattainable goal (Carver \& Scheier, 1990; Wrosch et al., 2007).

Wrosch and his colleagues (2003) found that compared with parents of children diagnosed with cancer who had poor goal adjustment capacities (both goal disengagement and goal reengagement), those with good goal adjustment capacities showed significantly lower levels of depression. Parents who had difficulties with both disengaging from unattainable goals and finding new goals were found to have the highest levels of depression. In a similar vein, Heckhausen, Wrosch and Fleeson (2001) 
found that women who run out of time in respect to childbearing (because of their biological clock), were better off if they could let go of their desire to have children and focus instead on other goals. These findings suggest that the capacity to disengage from unattainable goals and to engage in new purposeful activities is a protective factor that could mitigate the distress associated with the continued pursuit of unattainable goals.

Nolen-Hoeksema and colleagues (1994) studied the coping behaviors of bereaved adults. Ruminative coping, which is manifested in excessive worrying about one's depressive symptoms (but without a deliberate effort to address it in a constructive way), could be associated with bereavement. Bereaved individuals who ruminate about their predicament, may exacerbate their grief-related depression and experience a prolonged period of distress. While the loss of a loved one can be a devastating and overwhelming experience, those who can regain a positive outlook and find the strength to go on with life and find renewed meaning, appear to recover better than those who cope with their negative emotions through rumination. This is of interest in the context of mental illness, because parents often experience a bereavement-like phase when faced with a diagnosis of mental illness in a loved child. Moreover, while typical bereavement is associated with death and with a subsequent closure, bereavement in mental illness is further complicated by the lack of closure. Unlike the need to come to terms with the loss of a loved one, caregivers in mental illness are often faced with the 'death of a dream', rather than with the death of a loved one. The challenge of adjusting expectations and goals for their loved ones, as well as for themselves, may be an even more challenging task than accepting death.

Dunne, Wrosch, and Miller (2011) found that older adults suffering from 
functional disability experience smaller increases in depressive symptoms if they demonstrate good goal disengagement capacities. In the context of mental illness, caregiving often extends into old age and may subject the caregiver to the assaults of both old age and caregiving. Being able to adjust to unattainable goals, therefore, may be of an even greater importance among older caregivers.

Although the literature suggests that goal adjustment capacities can benefit a person's subjective well-being, the specific mechanisms that link goal adjustment capacities and indicators of subjective well-being have not yet been examined. Such an association could derive from the direct emotional benefits of avoiding the experience of failure and pursuing new meaningful goals. However, it is also possible that goal adjustment capacities serve other functions in the self-regulation of behavior that could influence subjective well-being. In this regard, they could facilitate the engagement in behavioral processes that are useful for effectively addressing the stressful circumstances that have rendered desired goals unattainable. This idea would be consistent with research among parents of children with cancer, which theorized that the emotional benefits of adaptive goal adjustment capacities could be due to the possibility that participants redirected resources to managing these stressful circumstances more effectively (Wrosch \& Scheier, 2003).

Such processes could contribute to the association between goal adjustment capacities and subjective well-being, and are generally referred to as coping. As mentioned earlier, coping typically includes cognitive and behavioral strategies that are activated to overcome problematic life circumstances, such as planning or problem solving. In addition, coping strategies are used to manage the emotional consequences of 
stressful encounters (Carver et al., 1989; Folkman, 1997; Lazarus \& Folkman, 1984). Some emotion-focused coping strategies, such as positive reframing, acceptance, or religious belief, can protect the self and contribute to meaning in life (Culver, Arena, Antoni, \& Carver, 2002; Folkman, 1997; Folkman \& Lazarus, 1988), particularly if a problem is unlikely to be resolved (Heckhausen et al., 2010). However, other emotionfocused coping strategies, such as denial, substance use, or venting, are often less useful and have been associated with maladaptive outcomes (Carver et al., 1989; Culver et al., 2002; Folkman \& Lazarus, 1988).

It is suggested that goal adjustment capacities may be associated with specific coping strategies, and that such an association is particularly likely to occur if individuals confront stressful life circumstances, such as caring for a child. In such situations, individuals may need to focus their time and energy on managing the stressor, which may result in the experience that the attainment of other desired goals has become impossible, such as career development, going on vacation, or buying a new car (Wrosch \& Scheier, 2003). Thus, individuals who tend to persist in the pursuit of unattainable goals may feel that they could have directed more energy at addressing the stressor and blame themselves for recurring problems. In addition, they are likely to deplete their selfregulation resources, which could interfere with managing the stressful circumstances (Baumeister, Bratslavsky, Muraven, \& Tice, 1998). Such a lack of self-regulation of resources could contribute to problems with active coping, but also to maladaptive behaviors associated with the urge to respond to emotionally difficult circumstances, such as problematic interpersonal behaviors (DeWall, Baumeister, Stillman, \& Gailliot, 2007). By contrast, individuals who are able to disengage from unattainable goals may use their 
resources more effectively to address the stressful life circumstances. Moreover, they may be less likely to engage in maladaptive coping strategies, and even if overcoming the stressor proves to be difficult, they may not blame themselves for occurring problems.

In a similar vein, it is also possible that individuals who have an easier time identifying and pursuing new goals are more successful at appropriately replacing the goals that have become unattainable due to a chronic stressor. This process could free resources needed to address the stressor and alleviate the perceived impact of the stressor on a person's life, thus contributing to a more positive evaluation of a problematic situation (Folkman, 1997; Folkman \& Moskowitz, 2000). By contrast, individuals who cannot identify new goals may feel that they lost control and thus have a difficult time accepting the stress-related life circumstances, and engage in maladaptive coping to regulate their emotions. However, it is noted that there is also some evidence suggesting that individuals can become stretched too thin if they pursue too many goals, which could have negative repercussions on the management of stressful life circumstances. For example, research has shown that adults experience high levels of negative affect about their regretted behaviors if they pursue many alternative goals, supposedly because they do not have sufficient resources to successfully address their regrets (Wrosch, Bauer, \& Scheier, 2005). Thus, goal reengagement could also be associated with a lack of selfregulation resources and maladaptive coping strategies, which could compromise levels of subjective well-being.

\section{Dispositional Optimism}

The literature on optimism refers to different schools of thought, viewing optimism alternately as a defense mechanism, an unrealistic illusion, a disposition, or a 
learned capacity (Peterson, 2000). For the purpose of this study, optimism is viewed as a cognitive variable describing generalized belief in good outcomes, based on a rational assessment of the likelihood of a successful outcome and a belief in personal efficacy (Scheier \& Carver, 1987). In that regard optimism is viewed as a personality trait that is relatively stable across time and context and is related to ego strength and internal control (Scheier \& Carver, 1985, 1992; Scheier, Carver, \& Bridges, 1994). Optimistic people expect positive outcomes and believe that good things will happen. They are better able to cope with challenges because they are both goal oriented and more flexible and constructive thinkers. When faced with an obstacle, such people tend to disengage themselves temporarily in order to assess the situation. Subsequently, they either continue to pursue the goal if they view it as attainable, or accept that it is unattainable and substitute a more realistic goal. Identifying goals and regulating activities to address these goals are the basis for the self-regulation model (Scheier \& Carver, 1985).

Optimism has an important impact on people's lives: it influences the way they face problems and cope with adversity, and it is associated with their social and socioeconomic resources (Carver et al., 2010). Optimism is associated with a reduced risk for psychopathology, and is inversely related to hopelessness and depression (Alloy et al., 2006). Moreover, optimism is linked with resiliency to stress, thus serving as a protective factor for mental and physical health (Lorant et al., 2003). A large body of research suggests that optimists not only use more adaptive ways to cope with stressful circumstances, but that their positive outlook provides benefits even in the absence of stress in both the intrapersonal and interpersonal spheres (Carver et al., 2010). Optimism, therefore, is not only linked to better emotional well-being, more effective coping 
behaviors, and potentially better physical health; it also translates into better relations with others and being better liked (Brissette, Scheier \& Carver, 2002). Playing an important role in the human experience, optimism is an individual difference variable that compels us to understand the mechanisms and processes that fuel it and determine the extent to which they can be taught to pessimists (Carver et al., 2010).

Constructs conceptually related to optimism have often been treated in empirical work as outcome variables rather than causes or stable dispositions. Scheier and Carver (1985) hypothesized that optimism has important implications for the way people regulate their behaviors, and proposed it may have important health-related consequences. They suggested that if outcome expectancies are favorable, individuals are likely to put more effort into their activity; however if outcome expectancies are not favorable, they are likely to reduce their efforts and may disengage completely from their attempt to attain the goal. Optimists may try harder than pessimists to attain their goals, or they may confront problems earlier than pessimists thus increase the likelihood of effective coping.

The benefits of dispositional optimism to health and well-being have been widely documented (Scheier, Carver, \& Bridges, 2001; Vaughn \& Leff, 1976). Cross-sectional and longitudinal studies demonstrated the association between optimism and subjective well-being (Khoo \& Bishop, 1997) self esteem (Dunn, 1996) and life satisfaction (Chang, 1998) as well as with low depression (Carver \& Gaines, 1987) and with low negative affect (King, Rowe, Kimble, \& Zerwic, 1998). The consistent and strong association between optimism and subjective well-being suggests that optimists may be using different strategies to cope with life adversities than pessimists do (Scheier et al., 1985). 
Optimists tend to use more active and complex strategies, including seeking social support, and are less engaged in emotional expression and disengagement from goals. Billings and Moos (1984) found that coping focusing on emotional expression was associated with dysfunction. Combining the two studies, it seems that optimists tend to use the most adaptive and least dysfunctional coping strategies, a finding consistent with Scheier and Carver's study (1985). Scheier and colleagues (1985) also found that when stressful situations were perceived as controllable, optimists tended to use problemsolving strategies; however, when the stressful situations were viewed as uncontrollable, optimists tended to reframe the situation in a more positive way, thus lightened up the burden.

In a study on recovering from coronary artery bypass surgery, Scheier and his colleagues (1989) found that compared with pessimists, optimistic men used more problem-solving coping behaviors and less denial than pessimists who were prone to worrying and focus on the negative aspects of their experience before the surgery. Optimists were more likely to make plans and set post-surgery goals. And while pessimists tended to avoid thinking about their experience, optimists actively sought information that could help them in their recovery. Additionally, optimists' physical recovery was faster and they returned to their pre-surgery life more quickly than pessimists. Moreover, the quality of life of optimists was superior to that of pessimists six months after the surgery. These findings suggest that optimism exerted a strong effect on the physical well-being and rate of recovery of the participating patients through coping strategies.

Gottlieb and Rooney (2004) recognize that positive beliefs about coping 
effectiveness represent an important psychological resource (Lazarus \& Folkman, 1984), and that such beliefs about progress towards achieving goals have a self-regulatory function (Carver \& Scheier, 1998). They attempt to clarify the relations between coping effectiveness and selected variables (both behavioral and dispositional) in the context of caregiving. Additionally they investigate the relations between caregivers' assessment of their own coping effectiveness, affect and general mental health. The researchers aimed to determine whether the impact of coping effectiveness on affect and mental health is exerted directly as a main effect, or indirectly as a moderating effect. They distinguish between optimism (viewed as personality disposition related to favorable outcomes) and self-efficacy (related to domain specific outcome expectancy), and try to establish which of these variables influence self-evaluation of coping effectiveness. They found a substantial and relatively equal influence of both dispositional optimism and self-efficacy on coping effectiveness, as assessed by the caregiver. As well, optimism was found to have a strong and consistent impact on outcome expectancies and on coping behaviors (Gottlieb \& Rooney, 2004). These findings suggest that optimism, as a personality construct, plays an important role in the stress process, either by improving coping outcomes, by fostering more positive coping evaluation, or by biasing the interpretation of coping (Klaaren, Hodges, \& Wilson, 1994). Additionally, Gottlieb and Rooney (2004) found that better mental health was associated with the employment of the coping behaviors of supportive symptom management and emotional inhibition, as well as with the caregivers' perception of the efficacy with which they handle stressful demands. Hulbert and Morrison (2006) found that optimism, more than self-efficacy, was consistently and strongly associated with low levels of perceived stress in caregivers 
within a palliative setting. These findings are consistent with previous studies that showed a strong buffering effect of optimism on caregiver stress. The greater buffering effect of optimism as compared with self-efficacy supports the observation that while some individuals can be generally optimistic, they can also be less self- efficacious when it comes to specific tasks.

Lyons and colleagues (2004) explored optimism and pessimism as potential predictors of negative changes in depressive symptoms and physical health of caregivers for spouses diagnosed with Parkinson's disease. High optimism was linked to better physical health in caregivers, and low optimism was associated with greater depression (Christensen, Stephens, \& Townsend, 1998; Given et al., 1993). This longitudinal study found that high pessimism early in the caregiver's role may be a warning sign for poor health of the caregiver both at the time of the study and in the future. They also found that the average caregiver attending to a spouse with Parkinson's disease over a 10-year period is at an increased risk for negative health, with significant change in both depressive symptoms and physical health. These findings may be relevant for caregiving in mental illness, since both diagnoses present a long-term challenge for caregivers. Moreover, their clinical implications are important for both caregiver and care recipient, since better supported caregivers are likely to provide better care for ill relatives.

\section{Unmitigated Communion}

Helgeson and Fritz (2000) describe agency as a construct that reflects focusing on the self, and communion as a construct focusing on other. Both concepts reflect a positive focus. It is when individuals manifest difficulties in relationship and poor health behaviors that agency may become unmitigated agency and communion may become 
unmitigated communion. Bakan (1966) first described communion as an important psychological variable, and while he never explicitly identified unmitigated communion as a personality construct, he suggested that a high level of communion must be mitigated by a personal sense of agency. High communion and low agency, thus, may be associated with the development of unmitigated communion.

Unmitigated agency - a narrow aspect of agency - reflects a focus on self to the exclusion of others, thus excludes communion. Both agency and unmitigated agency focus on the self, but while agency is a positive focus, unmitigated agency is characterized by excessive focus on the self and is associated with negative consequences. Unmitigated communion, on the other hand, is a narrow aspect of communion, which excludes agency. Unlike communion, unmitigated communion can have negative implications for both the individual themselves and their relations with others.

Both communion and unmitigated communion are associated with providing help to others; however people high on communion may have a genuine interest in others' well-being, while those high on unmitigated communion may be doing so in order to strengthen their own sense of worth through the eyes of others (Batson, 1995). In both unmitigated agency and unmitigated communion the person is reluctant to seek help from others. In the case of unmitigated communion, individuals tend to subjugate their own needs to the needs of others and are dependent on others for esteem; in the case of unmitigated agency individuals' negative views of others impede their willing to attend to relationships (Helgeson \& Fritz, 2000). 
The social interactions with others of high unmitigated communion individuals may be perceived as over-protective and intrusive, thus give way to conflicts and difficult relations. At the same time these individuals are reluctant to seek help from others because: they don't want to burden others with their own problems; they don't feel they deserve others' time and attention; they think others are not available for help (which may also be associated with poor self-esteem), or because they are uncomfortable receiving support (Fritz \& Helgeson, 1998). Essentially, Helgeson and Fritz (2000) suggest that by not seeking help from others, the unmitigated communion individuals can sustain a sense of importance and indispensability, which feeds their self-esteem. Individuals high on unmitigated communion however, exercise poor health behaviors as a side effect of their over-involvement with others, not as a deliberate intention (Helgeson \& Fritz, 2000).

Unmitigated communion is viewed as a personality trait because it is relatively stable over time (Helgeson \& Fritz, 1998). Helgeson and Fritz suggest that it likely develops from some combination of genetics and socialization, and is an interaction style that could be changed to some degree. When female subjects were asked what is the origin of unmitigated communion in their view, the most frequent answer was the way one was raised (38\%), followed by modeling of a family member, usually the mother (19\%), lack of self esteem (16\%), and genetics (13\%) (Helgeson \& Fritz, 1998). Women may be at risk for the development of unmitigated communion because they are socialized to have high regard for relationships and are encouraged to be involved in relationships (Miller, 1976), and because women are more susceptible to negative self perception than men (Lenney, 1977). Consistent with this argument, Bakan (1966) 
identified agency as the male principle and communion as the female principle of human existence. This distinction is relevant for the understanding of sex differences in behaviors and outcomes in various domains, such as relationships and health (Helgeson \& Fritz, 1996, 1999).

The combination of an orientation towards others together with negative self perception may lead more women than men to over-involvement with others as a way of raising self esteem. Low self esteem, therefore, may be an antecedent condition of unmitigated communion as well as its consequence. Additionally, poor family environment or unsatisfactory relationships early in life may also be associated with the development of unmitigated communion (Helgeson \& Fritz, 1998).

Unlike communion, unmitigated communion has been found to be associated with compromised psychological and physical well-being. Generalized psychological distress stems from one's cognitive perception of self, the over-involvement with others, and the neglect of one's own needs. Over-involvement with others exposes the individual to more problems of others and could therefore lead to situation-specific distress; if this leads also to the neglect of one's own needs, it may result in physical health problems as well (Helgeson \& Fritz, 1998).

Unmitigated communion was also found to be associated with anxiety and with depressive symptoms in various populations (Fritz \& Helgeson, 1998); for example it predicted depressive symptoms in longitudinal studies of people with chronic illnesses (Helgeson \& Fritz, 1996). One reason for the association of unmitigated communion with distress is the generally compromised self worth. Fritz and Helgeson (1998) found that a negative view of oneself and reliance on others for a sense of value was the reason that 
unmitigated communion is associated with distress. Self-esteem, in turn, was found to mediate the relation of unmitigated communion to depressive symptoms, but unmitigated communion did not mediate the relation of self-esteem to depressive symptoms (Fritz \& Helgeson, 1998). This supports earlier studies that demonstrated the consistent effect of low self-esteem on depressive symptoms (Taylor \& Brown, 1988).

In sum, unmitigated communion can be understood in terms of viewing one self, others, the relationships with others, as well as one's own well-being, both psychologically and physically. While communion is characterized by balanced, reciprocal and satisfying relationships, unmitigated communion is associated with heavy investment in relationships that does not lead to satisfying results. Because relationships are used as a way to enhance poor self-esteem, over-involvement is typically observed, associated with neglect of self, and leading to both psychological distress and physical symptoms. Self neglect may occur because the person has no time for self, because they don't feel deserving of paying attention to their needs, or because they believe that attending to own needs might compromise their relationships with others (Helgeson \& Fritz, 1998).

Examining unmitigated communion in the context of caregiving in mental illness is intriguing, because caregivers sometimes appear to compromise their own needs in order to attend to their ill relatives. They often argue that they do so out of necessity and not out of choice; for example, because of a shortage of services. However, it may well be that not only the circumstances dictate such behavior but also a personal tendency that may or may not be reinforced by circumstances. 
Unmitigated communion, therefore, may exacerbate the caregiving experience. Helping to extreme, being overly nurturing, intrusive, and/or self-sacrificing, may be not only counter-productive from the ill person's perspective, but also affect the caregiver's general well-being. These may lead to an imbalanced relationship, where caregivers provide support but don't necessarily receive support, possibly as a way of exercising control over the relationships and maintaining their identity as support providers (Helgeson \& Fritz, 1998). In that way, unmitigated communion could predict maladaptive coping and may compromise well-being.

To summarize, this study will examine goal adjustment capacities, dispositional optimism and low unmitigated communion, and whether they could play adaptive roles in the coping behaviors and subjective well-being of individuals who provide care for a family member with mental illness. In this regard, it is important to note that these personality factors represent independent constructs, which raises the possibility that there may be different groups of caregivers with different personality profiles (e.g., optimists who are able to disengage or optimists who have difficulty disengaging from unattainable goals). Because such profiles could further influence the way caregivers react and the ensuing levels of subjective well-being, this study will also explore the effects of different personality profiles. In this regard, two different ideas will be explored. First the accumulation of adaptive (or maladaptive) personality profiles could have particularly strong effects on coping and subjective well-being. Second, it seems plausible that adaptive personality factors could also compensate for the adverse effects of maladaptive personality factors. For example, high optimism could buffer the effect of difficulty with adjusting unattainable goals on a caregiver's depressive symptomatology. 


\section{Chapter 2}

\section{THE RESEARCH}

\section{Theoretical Model}

In order to explore how long-term caregiving to a mentally ill relative affects the subjective well-being of the caregiver, the functional associations between personality constructs, coping behaviors, and subjective well-being were examined. Assuming that caregiving to a family member with mental illness represents a chronic stressor (that could, for example, render desired goals unattainable), it was postulated that better subjective well-being will be present among caregivers with certain personality constructs. It was also expected that certain coping behaviors may be associated with some personality constructs, or with the profiles they create, more than with others.

To conceptualize the role of caregiver burden in the pathways linking personality constructs, or the profiles they create, with coping strategies and with general subjective well-being, two different possibilities were suggested as a possible explanation for how these constructs, that is, goal adjustment capacities, dispositional optimism and unmitigated communion, along with caregiver burden, can work together to influence subjective well-being. Drawing on the previously discussed mechanisms of personality associated with exposure and reactivity (Bolger \& Zuckerman, 1995), the first scenario suggests that caregiver burden could mediate the associations between adaptive personality traits and a person's general subjective well-being. In this scenario, adaptive personality traits could promote effective coping with caregiving stress, which in turn is likely to reduce caregiver burden and increase levels of subjective well-being.

In addition, at times, levels of caregiver burden can be somewhat independent 
from the person's adaptive personality traits or coping strategies. Such variability could occur if caregivers with generally adaptive personality traits confront circumstances in which they experience particularly elevated levels of burden. For example, a family member may display symptomatic behaviors or a caregiver is confronted with additional stressors in other domains of life. Given that elevated levels of caregiver burden can adversely affect subjective well-being (Grunfeld et al., 2004; Schulz et al., 1997), it seems that, during times of high burden, certain personality traits or adaptive personality profiles can become paramount for contributing to a caregiver's subjective well-being. This implies that these personality constructs and their associated coping strategies may also exert a buffering or moderating effect, helping to prevent the adverse spillover effect that caregiving burden can have on a person's general subjective well-being. Thus, it is theoretically possible that caregiver burden can either mediate the association between adaptive personality traits and subjective well-being or that adaptive personality traits and coping could moderate the link between high caregiver burden and low subjective wellbeing. Moreover, it is suggested that both scenarios do not necessarily exclude each other. While low caregiver burden may generally mediate the associations between adaptive personality and subjective well-being, variability in personal adaptability could produce additional buffering effects on the subjective well-being of highly burdened caregiver.

\section{The Present Research}

The goal of the thesis was to better understand the subjective experience of family caring for a mentally ill relative, by exploring the relationships between caregiver burden, selected personality constructs and various coping behaviors. Exploring individual 
differences aimed at understanding how such differences may contribute to, mitigate, or buffer the burden experience, and how, ultimately, burden and the coping behaviors associated with it may influence subjective well-being.

Chronic burden is known to have important impact on subjective well-being. Thus family caregivers' well-being is likely to be compromised by the chronic burden and stress they experience in their role as caregivers. Additionally, individual differences may play an important role in how people respond to life circumstances and thus affect their subjective well-being.

The research, therefore, examined the associations between selected personality constructs, chronic stress and burden, coping strategies and indicators of subjective wellbeing in a sample of adult family members who provide unpaid care to a family member diagnosed with a mental illness.

The selected personality constructs include goal adjustment capacities, dispositional optimism, and unmitigated communion. The indicators of subjective wellbeing include symptoms of depression, satisfaction with life, purpose in life, as well as positive and negative affect.

It was expected that individual differences in goal adjustment capacities, that is, goal disengagement and goal reengagement capacities, as well as dispositional optimism and unmitigated communion, would predict different baseline levels of, and different changes over time, in stress-specific and general indicators of subjective well-being, that is depressive symptoms, purpose and satisfaction with life, positive and negative affect. In this regard, the effects of goal adjustment capacities, optimism, and unmitigated communion on general and specific indicators of subjective well-being were explored, in 
order to determine if they can be mediated by caregiver burden or whether these constructs can buffer the associations between caregiver burden and subjective wellbeing. Additionally, coping behaviors were examined in order to determine whether they could qualify as mediators and statistically explain the cross-sectional and longitudinal effects of the selected personality constructs on indicators of subjective well-being. Both optimism and goal adjustment capacities already appear to be associated with positive consequences in the face of challenging life situations. Moreover, the association between optimism and the choice of coping behaviors has been already established (Scheier et al., 1985; Wrosch \& Scheier, 2003), leading to the assumption that similar associations may exist between other personality traits and coping.

To avoid spurious associations, the hypotheses were evaluated by statistically controlling the analyses for a number of covariates that have been shown in previous research to predict caregiver burden, self-regulation constructs, or subjective well-being. The covariates included participants' age, sex, educational level, partnership status, chronic health problems, and their reports of the relative's illness severity, as well as the years since their relative's first diagnosis (see Pinquart \& Sorensen, 2003; Pratt et al., 1985; Wrosch \& Scheier, 2003).

Because of differences in opinion regarding alpha level correction for multiple comparisons (e.g., Perneger, 1998), $p$-value $<.05$ was considered significant. While this strategy may further be appropriate, given that the study's hypotheses are theory based and directed, results should nonetheless be interpreted with caution. 


\section{Hypotheses}

It is hypothesized that in the context of caregiving for a family member diagnosed with mental illness, different personality constructs and the personality profiles created through the interactions between personality constructs may facilitate certain carespecific coping behaviors, and consequently influence the caregivers' experience and subjective well-being. More specifically it is hypothesized that:

1. Personality constructs are associated with caregiver burden and subjective wellbeing outcomes. Especially, high levels of goal adjustment capacitiesdisengagement and reengagement- and optimism, and low levels of unmitigated communion are expected to be associated with less caregiving burden and better subjective well-being outcomes, and to predict declines in caregiving burden and increases in subjective well-being outcomes over time.

2. These adaptive personality constructs are also expected to be associated with higher levels of adaptive care-specific coping behaviors.

3. Care-specific coping behaviors are further expected to be associated with levels and changes in the caregiver's experience of burden and subsequent subjective well-being outcomes: adaptive coping is likely to mitigate caregiver burden and predict better well-being.

4. There are two pathways by which caregiving burden and personality could influence the associations between personality constructs and subjective wellbeing:

a. Caregiver burden could mediate the associations between adaptive personality and subjective well-being. In this scenario adaptive personality 
constructs can promote effective coping with caregiver stress and predict levels and changes in caregiver burden and subjective well-being.

b. Adaptive personality constructs and the coping behaviors they activate can buffer, or moderate, the adverse effects of caregiver burden on levels and changes in indicators of subjective well-being, thereby preventing a possible spillover effect of caregiver burden on subjective well-being.

5. Personality profiles, that are formed through interactions between personality constructs, are associated with burden and affect subjective well-being in two ways:

a. Combinations of adaptive personality constructs are likely to be associated with higher levels and increases in well-being outcomes, and combinations of maladaptive personality constructs - with lower levels and declines in well-being outcomes.

b. Adaptive personality constructs may compensate for maladaptive personality constructs and thus prevent the adverse effects of maladaptive personality factors on levels and changes in caregiver burden and subjective well-being.

\section{Method}

\section{Participants and Procedures}

One hundred and fifty three caregivers of family members with mental illness were recruited into the study in 2008. Letters were sent to the membership of AMIQuebec Action on Mental Illness, which is an independent and government-supported organization that provides support to predominantly English speaking families in Quebec, 
Canada, who are faced with mental illness. The study was approved by the board of directors of AMI-Quebec, and Concordia University's human research ethics board. Questionnaire packages were sent to AMI members who agreed to participate in the study. Of the 153 participating, 6 were excluded from the study because their relatives were either deceased or they misunderstood the instructions and did not produce usable data. Approximately 17 months $(M=16.80, S D=1.24)$ after the baseline assessment, participants were contacted again and asked to respond to another questionnaire. One hundred twenty one of the original 147 caregivers participated in the follow-up (82\%). Study attrition was not significantly associated with most of the study's baseline measures of depressive symptoms, purpose, caregiver burden, 12 of the 14 coping strategies, goal adjustment capacities, chronic illness, caregivers' age, sex, education, and partnership status, and caregivers' reports of their relatives' illness severity and time since first diagnosis. However, participants who dropped out of the study reported significantly lower baseline levels of using instrumental support $(M=3.04, S D=1.82)$ and acceptance $(M=3.85, S D=1.57)$ to cope with caregiving stress, compared with their counterparts who continued their participation (instrumental support: $M=3.82, S D=$ 1.61 ; acceptance: $M=4.47, S D=1.36 ; t(145)=2.06, p=.04)$.

At baseline, the 147 participants were on average 60.73 years old $(S D=12.35)$, $78 \%$ were female, $57 \%$ received an undergraduate degree or higher (masters or doctorate $=22 \%$, bachelor $=35 \%$, trade school $=26 \%$, high school $=15 \%$, grade school $=1 \%$, and $73 \%$ were married (65\%) or cohabitating with a partner $(8 \%)$. Sixty six percent of the participants were parents caring for an adult child; $17 \%$ were spouses, $14 \%$ cared for a sibling and $3 \%$ were caregivers to a parent with mental illness. According to the 
caregivers' reports, their relatives were first diagnosed with a mental illness, on average, 15.73 years ago $(S D=11.03)$. Forty-one percent of their relatives were diagnosed with schizophrenia, $37 \%$ with a mood disorder, and $22 \%$ with other mental disorders (OCD, ADHD, mixed diagnosis, or unknown).

\section{Materials}

The main study variables included repeated measures of participants' depressive symptoms, purpose in life, satisfaction with life, positive and negative affect, and caregiver burden. In addition, goal adjustment capacities, optimism, unmitigated communion and coping with caregiving stress, as well as relevant covariates (caregivers' age, sex, education, partnership status, and chronic health problems, and perceptions of their relatives' illness severity and time since first diagnosis) were assessed at baseline.

Depressive symptomatology was measured with the 20-item Center for

Epidemiological Studies Depression Scale (CES-D, Radloff, 1977). Participants were asked to rate how frequent they had experienced each of twenty depressive symptoms during the past week on 4-point Likert-type scales $(0=$ less than one day, $3=5-7$ days $)$. Sample items included: I felt depressed or I felt that everything I did was an effort. Sum scores of the twenty depressive symptoms were computed separately for baseline, $M=$ 9.76, $S D=9.16, \propto=.90$, and follow-up, $M=9.82, S D=8.91, \propto=.89$. Depressive symptoms were significantly correlated across measurements, $r=.62, p<.01$, and did not change significantly over time, $t(120)=1.36, p=.17$.

Purpose in life was assessed by administering the previously validated Life Engagement Test (Scheier et al., 2006). This scale consists of 6 items measuring the extent to which people engage in personally valued activities. Participants were asked to 
indicate their level of agreement with these items, using 5-point Likert-type scales $(1=$ strongly disagree, $5=$ strongly agree . Sample items included: There is not enough purpose in my life or I have lots of reasons for living. Sum scores of the six items were computed separately for baseline, $M=25.16, S D=3.71, \propto=.81$, and follow-up, $M=$ 25.08, $S D=3.28, \propto=.72$. High scores on the scale indicated high levels of purpose in life. Purpose in life was correlated across assessments, $r=.61, p<.01$, and did not change significantly over time, $t(120)=-1.07, p=.29$.

Life satisfaction was assessed by administering the previously validated Satisfaction With Life Scale (SWLS, Diener et al., 1985). This scale consists of 5 items measuring global life satisfaction. Participants were asked to indicate their level of agreement with these items, using 5 -point Likert-type scales $(1=$ strongly disagree, $5=$ strongly agree). Sample items included: In most ways my life is close to my ideal or I am satisfied with my life. Mean scores of the six items were computed separately for baseline, $M=2.29, S D=.83, \propto=.88$, and follow-up, $M=2.31, S D=.76, \propto=.86$. High scores on the scale indicated high levels of satisfaction with life. Satisfaction with life was correlated across assessments, $r=.75, p<.01$, and did not change significantly over time, $t(120)=-.36, p>.05$.

Positive and negative affects were assessed by administering the previously validated Positive and Negative Affect Schedule (Watson et al., 1988). This scale consists of two 10-item mood scales measuring both positive and negative affect. Participants were asked to indicate the extent to which they experienced the different emotions during the past year, using 5 -point Likert-type scales $(1=$ strongly disagree, $5=$ strongly agree $)$. Sample items included: interested or enthusiastic (positive affect); distressed or ashamed 
(negative affect). Mean scores of the ten items were computed for each scale separately for baseline; positive affect: $M=2.66, S D=.65, \propto=.88$; negative affect: $M=1.40, S D$ $=.85, \propto=.90$, and follow-up, positive affect: $M=2.60, S D=.64, \propto=.88$; negative affect: $M=1.19, S D=.75, \propto=.89$. High scores on the scales indicated high levels of affect (positive or negative). Both positive affect and negative Affect were correlated across assessments: positive affect: $r=.77, p<.01$, and negative affect: $r=.64, p<.01$. Positive affect did not significantly change over time, $t(120)=-1.65, p>.05$; negative affect declined significantly over time, $t(120)=-2.45, p<.05$.

Goal adjustment capacities were measured with the previously validated 10-item Goal Adjustment Scale (Wrosch et al., 2003). Participants were asked to report how they usually react when they have to stop pursuing an important goal in their life. Four items measured participants' goal disengagement capacities (e.g., It's easy for me to reduce my effort towards the goal or I stay committed to the goal for a long time; I can't let it go), and six items measured their goal reengagement capacities (e.g., I seek other meaningful goals or I start working on other new goals). Responses were measured on 5-point Likert-type scales, ranging from $1=$ strongly disagree, to $5=$ strongly agree . Mean scores were computed at base line separately for goal disengagement, $M=2.81, S D=$ $.79, \propto=.75$, and goal reengagement, $M=3.52, S D=.78, \propto=.91$. High scores indicated that participants had an easier time disengaging from unattainable goals and reengaging in new goals. Goal disengagement and goal reengagement capacities were not highly correlated with each other, $r=.33, p<.01$.

Optimism was assessed by administering the previously validated Revised Life Orientation Test (Scheier et al., 1994). This scale consists of 10 items measuring general 
outcome expectancies. Participants were asked to indicate their level of agreement with these items, using 5-point Likert-type scales $(1=$ strongly disagree, $5=$ strongly agree $)$. Sample items included: In uncertain times, I usually expect the best, or I am always optimistic about the future. Mean scores of the ten items were computed for baseline, $M$ $=2.80, S D=.62, \propto=.76$. High scores on the scale indicated high levels of optimism. Optimism was correlated across assessments, $r=.74, p<.01$, and did not change significantly over time, $t(117)=.77, p>.05$.

Unmitigated communion was assessed by administering the previously validated Unmitigated Communion Scale (Helgeson \& Fritz, 1998). This scale consists of 9 items measuring involvement with others. Participants were asked to indicate their level of agreement with these items, using 5-point Likert-type scales $(1=$ strongly disagree, $5=$ strongly agree). Sample items included: I always place the needs of others above my own, or I can't say no when someone asks me for help. Mean scores of the nine items were computed for baseline, $M=2.59, S D=.68, \propto=.79$. High scores on the scale indicated high levels of unmitigated communion. Unmitigated communion was correlated across assessments, $r=.74, p<.01$ and did not change significantly over time, $t(120)=-.64, p>.05$

Caregiver burden was assessed using the 22-item Burden Interview (BI; Zarit \& Zarit, 1987), which is a widely used measure of burden among caregivers. The wording of the original items was slightly adjusted, in an attempt to make them more specific to caregiving for relatives with mental illness (e.g., How often do you feel strained when you are with your relative? or How often do you feel that your relative is dependent on you?). The twenty-two items were answered by the participants, using 5-point Likert-type scales 
(endpoints: $0=$ never $; 4=$ nearly always), and sum scores of the 22 items were computed. Higher values indicated greater caregiver burden. The average level of caregiver burden in the current sample was $32.88(S D=15.33 ; \propto=.93)$ at baseline and $30.89(S D=14.61, \propto=.93)$ at follow-up. Caregiver burden was significantly correlated across measurements, $r=.75, p<.01$, and significantly declined over time, $t(120)=-$ 2.28, $p=.02$. Compared to normative Canadian data of elderly dementia caregivers, the average baseline level of burden in the current sample can be characterized as high to severe burden (i.e., $75^{\text {th }}$ percentile, Hébert, Bravo, \& Preville, 2000a).

Coping with stressful caregiving situation was measured by administering the Brief Cope (Carver, 1997). This scale was created to avoid time burden and impatience among vulnerable populations and represents an abbreviated version of the full Cope (Carver et al., 1989). The coping instrument included 28 items assessing 14 different coping strategies. Participants were asked to report whether they have been engaging in specific coping behaviors to manage the stress associated with caring for their relative with mental illness. The coping strategies were measured with 4-point Likert-type scales (endpoints $0=$ I haven't been doing this at all, $4=I$ have been doing this a lot). For each of the 14 types of coping strategies, a sum score of the 2 associated items was computed at base line, which were significantly correlated with each other: Active coping (e.g., I take action to try to make the situation better, $r=.46, p<.01, M=4.50, S D=1.42)$, planning (e.g., I think hard about what steps to take, $r=.65, p<.01, M=4.40, S D=$ 1.63), use of instrumental support (e.g., I get help and advice from other people, $r=.71$, $p<.01, M=3.68, S D=1.67$ ), behavioral disengagement (e.g., I give up the attempt to cope, $r=.48, p<.01, M=.69, S D=1.11$ ), positive reframing (e.g., I try to see it in a 
different light, to make it seem more positive, $r=.47, p<.01, M=3.26, S D=1.73$ ), acceptance, (e.g., I learn to live with it, $r=.32, p<.01, M=4.36, S D=1.41$ ), self-blame (e.g., I blame myselffor things that happen, $r=.52, p<.01, M=1.46, S D=1.38$ ), humor (e.g., I make jokes about it, $r=.57, p<.01, M=1.39, S D=1.50)$, religion (e.g., I pray or meditate, $r=.82, p<.01, M=2.38, S D=2.28)$, use of emotional support (e.g., I get comfort and understanding from someone, $r=.51, p<.01, M=3.52, S D=1.70)$, selfdistraction (e.g., I turn to work or other activities to take my mind off things, $r=.34, p<$ $.01, M=3.71, S D=1.55)$, denial (e.g., I refuse to believe it has happened, $r=.38, p<$ $.01, M=.61, S D=1.13$ ), venting (e.g., I express my negative feelings, $r=.41, p<.01, M$ $=2.38, S D=1.45)$, and substance use (e.g., I use alcohol or other drugs to make myself feel better, $r=.76, p<.01, M=.49, S D=1.06)$.

Covariates: To avoid spurious associations, the study also incorporated a number of variables that were used as covariates in the analyses. In this regard, participants' age, sex, educational level, and partnership status was assessed at baseline. Moreover, participants' chronic health problems were assessed by counting the presence of 19 different chronic health problems (e.g., cancer, high blood pressure, arthritis, or heart disease, $M=1.88, S D=1.70)$. Participants also reported how many years ago their relative had their first diagnosis $(M=15.73, S D=11.03)$. Finally, participants rated their relative's illness severity by using a 5-point Likert-type scale, with 5 being the most severe form of illness $(M=3.16, S D=1.06)$ 


\section{Chapter 3}

\section{RESULTS}

\section{Analyses plan}

The results are presented in four sections. In the first section the effects of the covariates included in the analyses are described. The associations between covariates and baseline indicators of subjective well-being were explored through correlation analyses.

The second section focuses on coping as a mechanism that may link personality constructs with caregiver burden and subjective well-being. To explore the associations between care specific coping behaviors and the three selected personality constructs, as well as the associations between care specific coping behaviors and indicators of subjective well-being, two sets of partial correlations were computed.

In the third section the associations between the selected personality constructs and indicators of subjective well-being are described, along with the role caregiver burden and coping strategies may play in these associations. The associations between each of the selected personality construct, caregiver burden, and subjective well-being outcomes were explored through hierarchical regression analyses

The final section describes the effects of personality profiles that were formed through interactions between the selected personality constructs, and examines how these profiles are associated with caregiver burden, coping behaviors, and subjective wellbeing. Hierarchical regression analyses were used. 


\section{Covariates}

To explore the influence of covariates, evaluations were conducted by examining the associations between caregiver burden, personality constructs and subjective wellbeing. Covariates included participants' age, sex, educational level, partnership status, chronic health problems, perceived illness severity, and time since relative's first diagnosis. Covariates were further included in subsequent analyses.

Correlations between covariates and baseline and follow-up levels of indicators of subjective well-being are described in Tables 1 and 2 and reveal the following results: age was significantly associated with baseline levels of positive and negative affect but not with follow-up levels. Younger adults experienced more positive but also more negative affect than older adults. Compared with females, males reported significantly less depressive symptoms at follow-up but not at baseline analysis. Education was significantly associated with lower baseline levels, but not with follow-up levels of depressive symptoms. More educated participants experienced fewer depressive symptoms compared with less educated participants. Partnership status was significantly associated with baseline levels of satisfaction with life, purpose in life and positive affect: participants married or living with a partner reported higher baseline levels of purpose in life, satisfaction with life and positive affect, compared with participants who were single. They also experienced fewer depressive symptoms and lower burden than their single counterparts. Partnership status was also significantly negatively correlated with follow-up levels of depressive symptoms and significantly positively correlated with purpose in life. Illness severity was significantly correlated with baseline levels of depressive symptoms and burden: compared with less severe illness, illness perceived as 
more severe by the caregiver was associated with higher burden and more depressive symptoms. Chronic health problems were significantly and negatively associated with baseline levels of satisfaction with life and positive affect: caregivers experiencing more health problems also reported less satisfaction with life and less positive affect. Finally, there was a significant association between years since relative's first diagnosis and follow-up levels of depressive symptoms: a more recent diagnosis was associated with higher levels of depressive symptoms at follow-up. Covariates were used in all subsequent analyses, correlations, model testing and mediation effects. 
Table 1

Correlations between covariates and baseline indicators of subjective well-being

\begin{tabular}{|c|c|c|c|c|c|c|}
\hline & $\begin{array}{l}\text { Depressive } \\
\text { symptoms }\end{array}$ & $\begin{array}{c}\text { Satisfaction } \\
\text { with life }\end{array}$ & $\begin{array}{c}\text { Purpose in } \\
\text { life }\end{array}$ & $\begin{array}{l}\text { Positive } \\
\text { affect }\end{array}$ & $\begin{array}{l}\text { Negative } \\
\text { affect }\end{array}$ & $\begin{array}{c}\text { Caregiver } \\
\text { burden }\end{array}$ \\
\hline$\overline{\text { Age }}$ & -.14 & -.01 & -.06 & $-.17 *$ & $-.23 * *$ & -.07 \\
\hline $\operatorname{Sex}^{\mathrm{a}}$ & -.11 & .15 & .04 & -.03 & -.11 & -.09 \\
\hline Education & $-.21 *$ & .12 & .08 & .08 & -.07 & -.14 \\
\hline Partnership status ${ }^{\text {b }}$ & $-.31^{* *}$ & $.22 * *$ & $.25^{* *}$ & $.27^{* *}$ & -.11 & $-.21 * *$ \\
\hline Illness severity & $.19 *$ & -.12 & -.06 & -.06 & .13 & $.27^{* *}$ \\
\hline Chronic health & .10 & $-.18 *$ & -.13 & $-.18 *$ & .10 & .06 \\
\hline Years since $1^{\text {st }}$ diagnosis & -.05 & .14 & -.05 & -.04 & -.15 & -.10 \\
\hline
\end{tabular}

Note. ${ }^{\text {a }}$ Higher values represent female participants; ${ }^{b}$ Higher values represent participants married or living with a partner. Lower values - living alone. $N=147,{ }^{*} p<.05 ;{ }^{*} p<.01$ 
Table 2

Correlations between covariates and changes in indicators of subjective well-being (T2) controlled for baseline well-being (T1)

\begin{tabular}{|c|c|c|c|c|c|c|}
\hline & $\begin{array}{c}\mathrm{T} 2 \\
\text { Depressive } \\
\text { symptoms }\end{array}$ & $\begin{array}{c}\text { T2 } \\
\text { Satisfaction } \\
\text { with life }\end{array}$ & $\begin{array}{c}\mathrm{T} 2 \\
\text { Purpose in } \\
\text { life }\end{array}$ & $\begin{array}{c}\mathrm{T} 2 \\
\text { Positive } \\
\text { affect }\end{array}$ & $\begin{array}{c}\text { T2 } \\
\text { Negative } \\
\text { affect }\end{array}$ & $\begin{array}{c}\mathrm{T} 2 \\
\text { Caregiver } \\
\text { burden }\end{array}$ \\
\hline Age & -.01 & -.04 & -.01 & -.03 & .05 & -.07 \\
\hline $\operatorname{Sex}^{\mathrm{a}}$ & $-.20 *$ & -.09 & .06 & -.01 & -.07 & -.01 \\
\hline Education & -.18 & .09 & .12 & .04 & -.03 & .13 \\
\hline Partnership status ${ }^{\mathrm{b}}$ &.$- .30 * *$ & .11 & $.20 *$ & .10 & -.18 & -.12 \\
\hline Illness severity & -.10 & -.02 & -.02 & -.02 & -.03 & -.14 \\
\hline Chronic health & .15 & -.16 & -.14 & -.10 & .18 & -.00 \\
\hline Years since $1^{\text {st }}$ diagnosis & $-.28 * *$ & .07 & .18 & .02 & .14 & -.06 \\
\hline
\end{tabular}

Note. ${ }^{\text {a }}$ Higher values represent female participants; ${ }^{\mathrm{b}}$ Higher values represent participants married or living with a partner. Lower values - living alone. $N=121, * p<.05 ; * * p<.01$ 


\section{Coping}

It was expected that personality constructs may facilitate the use of various coping behaviors and that these coping strategies may mediate the associations between the selected personality constructs and indicators of subjective well-being. Behaviors included in the analyses represent a range of 14 strategies people often use to cope with stressful situations, such as seeking instrumental or emotional support, acceptance of a stressful situation or reframing it in a positive way, but also behaviors such as denial, self-blame, and the use of alcohol or drugs (see Method section). To explore the associations between care specific coping behaviors and the three selected personality constructs, as well as the associations between care specific coping behaviors and indicators of subjective well-being, two sets of partial correlations were computed. The results are presented in Table 3 (correlations between baseline levels of the 14 assessed coping strategies and the personality constructs) and Table 4 (correlations between baseline levels of the coping strategies and the baseline and follow-up levels of indicators of subjective well-being). All the analyses were controlled for participants' age, sex, education, partnership status, chronic illness, time since relative's first diagnosis, and illness severity.

The associations between care specific coping behaviors and goal adjustment capacities, dispositional optimism, and unmitigated communion

Associations between goal adjustment capacities and coping behaviors

The exploration of the associations between goal adjustment capacities and coping with caregiving stress (Table 3) showed that both goal disengagement and goal reengagement capacities were significantly associated with certain care-specific coping 
Table 3

Correlations between goal adjustment capacities, optimism, unmitigated communion and coping with caregiving stress

\begin{tabular}{|c|c|c|c|c|}
\hline Coping with stress & $\begin{array}{c}\text { Goal dis- } \\
\text { engagement }\end{array}$ & $\begin{array}{c}\text { Goal re- } \\
\text { engagement }\end{array}$ & Optimism & $\begin{array}{l}\text { Unmitigated } \\
\text { communion }\end{array}$ \\
\hline Active coping & -.07 & $.16^{*}$ & $.28 * *$ & .08 \\
\hline Planning & .00 & $.19 *$ & $.22 * *$ & -.01 \\
\hline Instrumental support & .03 & $.28 * *$ & .13 & .08 \\
\hline Behavioral disengagement & -.02 & -.03 & $-.26 * *$ & .02 \\
\hline Positive reframing & .07 & $.25 * *$ & $.36 * *$ & -.10 \\
\hline Acceptance & .07 & .14 & $.22 *$ & -.04 \\
\hline Self-blame & $-.23 * *$ & -.06 & $-.26 * *$ & .10 \\
\hline Humor & .04 & $.17 *$ & -.11 & -.01 \\
\hline Religion & .07 & $.27 * *$ & .17 & -.06 \\
\hline Emotional support & -.06 & .12 & .15 & -.06 \\
\hline Self -distraction & -.09 & $.21 * *$ & -.03 & -.04 \\
\hline Denial & -.05 & -.10 & $-.29 * *$ & -.00 \\
\hline Venting & -.03 & $.26 * *$ & -.10 & -.09 \\
\hline Substance use & $-.22 * *$ & $-.25 * *$ & $-.17 *$ & .03 \\
\hline
\end{tabular}

Note: Analyses were controlled for caregivers' age, sex, education, partnership status, and chronic health problems, and time since care recipients' first diagnosis and illness severity. $N=147,{ }^{*} p<.05 ;{ }^{* *} p<.01$. 
behaviors. Goal disengagement capacities were significantly associated with self-blame and substance use. Participants who had an easier time abandoning unattainable goals blamed themselves less frequently for problems associated with caregiving and used alcohol or drugs less frequently to regulate their emotions than their counterparts who had more difficulty with goal disengagement. Goal reengagement capacities were also associated with a number of different care-specific coping strategies. Participants with better goal reengagement capacities engaged more frequently in active coping, planning, use of instrumental support, positive reframing, humor, religion, self-distraction, and venting than participants with poor goal reengagement capacities. Substances, however, were more frequently used by participants with lower, as compared to higher, levels of goal reengagement capacities (see Table 3).

\section{Associations between dispositional optimism and coping behaviors}

The exploration of the association between dispositional optimism and coping with caregiving stress showed that optimism was significantly associated with a number of care-specific coping behaviors. More specifically, optimism was significantly associated with active coping, planning, positive reframing and acceptance: more optimistic caregivers coped with caregiving stress more frequently by actively seeking solutions, accepting their situation or reframing it in a positive way. Optimism was negatively associated with behavioral disengagement, self-blame, denial, and substance use: compared with more pessimistic caregivers, more optimistic caregivers persisted more on achieving their goals, blamed themselves less, and used denial or resorted to drugs or alcohol to a lesser degree (see Table 3). 
Associations between unmitigated communion and coping behaviors

No significant associations were found between unmitigated communion and care specific coping behaviors with caregiving stress (see Table 3).

The associations between care specific coping behaviors and baseline and follow- up levels of indicators of subjective well-being

Associations between care specific coping and caregiver burden

Baseline levels of caregiver burden were significantly associated with self-blame, humor, self-distraction, denial and venting (Table 4): participants reporting high burden also criticized themselves and took responsibility for problems related to their caregiving role; they had a hard time accepting their situation and tried to take their mind off their stress by turning to other activities. Follow-up levels of caregiver burden (T2), however, were associated only with higher baseline levels of self-blame and self-distraction. Associations between care specific coping and depressive symptoms

Depressive symptoms at baseline were significantly associated with behavioral disengagement, self-blame and denial: participants using denial, blaming themselves or giving up altogether, showed higher levels of depressive symptoms. However, lower levels of baseline depressive symptoms were associated with active coping, positive reframing, acceptance, religion and emotional support: participants taking action to improve a stressful situation, learn to live with it or trying to find some positive in it, as well as those getting emotional support from others or finding comfort in religion or spiritual beliefs showed less depressive symptoms. At follow-up (T2), depressive symptoms were positively associated with baseline levels of behavioral disengagement, 
self-blame and substance use, and negatively associated with positive reframing (Table 4).

Associations between care specific coping and purpose in life

At both baseline and follow-up, purpose in life was positively associated with active coping, planning, positive reframing, and acceptance, and negatively associated with behavioral disengagement, self-blame and denial. Baseline levels of purpose were also positively associated with instrumental support and religion; follow-up levels of purpose were positively associated with baseline levels of emotional support, and negatively associated with substance use. For example, participants choosing to accept a stressful situation, reframed it in a positive way, or actively coped by seeking help or comfort in religion, showed higher levels of purpose in life compared with participants who blamed themselves, used denial, or gave up (Table 4).

Associations between care specific coping and satisfaction with life

Baseline levels of satisfaction with life were positively associated with positive reframing and negatively associated with behavioral disengagement and self- blame. Follow-up levels of satisfaction with life were negatively associated with baseline levels of behavioral disengagement, self-blame, denial, venting and substance use, and positively associated with emotional support. For example, participants using positive reframing demonstrated a higher sense of satisfaction with life, while those using selfblame or behavioral disengagement reported lower levels of satisfaction with life (Table 4). 
Table 4

Correlations between baseline levels of coping with caregiving stress and baseline (T1) and follow-up (T2) levels of indicators of subjective well-being.

\begin{tabular}{|c|c|c|c|c|c|c|c|c|c|c|c|c|}
\hline \multirow[t]{2}{*}{ Coping with stress } & \multicolumn{2}{|c|}{$\begin{array}{l}\text { Caregiver } \\
\text { burden }\end{array}$} & \multicolumn{2}{|c|}{$\begin{array}{l}\text { Depressive } \\
\text { symptoms }\end{array}$} & \multicolumn{2}{|c|}{$\begin{array}{l}\text { Purpose } \\
\text { in life }\end{array}$} & \multicolumn{2}{|c|}{$\begin{array}{c}\text { Satisfaction } \\
\text { with life }\end{array}$} & \multicolumn{2}{|c|}{$\begin{array}{c}\text { Positive } \\
\text { affect }\end{array}$} & \multicolumn{2}{|c|}{$\begin{array}{l}\text { Negative } \\
\text { affect }\end{array}$} \\
\hline & $\mathrm{T} 1$ & $\mathrm{~T} 2$ & $\mathrm{~T} 1$ & $\mathrm{~T} 2$ & $\mathrm{~T} 1$ & $\mathrm{~T} 2$ & $\mathrm{~T} 1$ & $\mathrm{~T} 2$ & $\mathrm{~T} 1$ & $\mathrm{~T} 2$ & $\mathrm{~T} 1$ & $\mathrm{~T} 2$ \\
\hline Active Coping & -.02 & .05 & $-.19 *$ & .01 & $.29 * *$ & $.32 * *$ & .09 & .01 & $.29 * *$ & $.29 * *$ & -.08 & -.01 \\
\hline Planning & .08 & .15 & -.11 & .09 & $.30 * *$ & $.25 * *$ & .12 & .03 & $.18 *$ & $.25 * *$ & -.01 & .03 \\
\hline Instrumental support & -.01 & -.05 & -.09 & .04 & $.22 * *$ & .09 & .07 & .08 & .11 & $.23 * *$ & -.08 & .04 \\
\hline Behav' disengagement & .05 & .01 & $.27 * *$ & $.19 * *$ & $-.21 * *$ & $-.29 * *$ & $-.17 *$ & $-.20 *$ & -.15 & $-.22 *$ & $.24 * *$ & .14 \\
\hline Positive reframing & .02 & -.04 & $-.35 * *$ & $-.24 * *$ & $.40 * *$ & $.27 * *$ & $.26 * *$ & .17 & $.37 * *$ & $.25 * *$ & $-.29 * *$ & $-.28 * *$ \\
\hline Acceptance & -.15 & -.12 & $-.34 * *$ & -.15 & $.30 * *$ & $.21^{*}$ & .14 & .06 & $.26 * *$ & $.24 * *$ & $-.29 * *$ & $-.25 * *$ \\
\hline Self-blame & $.28 * *$ & $.22 * *$ & $.21 * *$ & $.26 * *$ & $-.21 * *$ & $-.20 *$ & $-.21 *$ & $-.26 * *$ & -.06 & $-.23 * *$ & $.44 * *$ & $.45 * *$ \\
\hline Humor & $.20 *$ & .10 & .01 & .11 & -.06 & -.07 & -.08 & -.08 & .03 & .10 & .04 & -.02 \\
\hline Religion & -.14 & -.08 & $-.21 * *$ & .01 & $.22 * *$ & .14 & .12 & .00 & $.19 *$ & .05 & -.10 & -.04 \\
\hline Emotional support & -.14 & -.11 & $-.28 * *$ & -.09 & .15 & $.19 *$ & .16 & $.22 * *$ & $.18^{*}$ & $.22 *$ & -.03 & .00 \\
\hline Self-distraction & $.23 * *$ & $.27 * *$ & .09 & .05 & -.08 & .05 & -.16 & -.15 & .14 & .09 & $.18 *$ & .05 \\
\hline Denial & $.21 * *$ & .08 & $.31 * *$ & .13 & $-.28 * *$ & $-.22 *$ & -.16 & $-.19 *$ & -.17 & $-.23 * *$ & $.26 * *$ & .08 \\
\hline Venting & $.23 * *$ & .16 & .05 & .08 & -.03 & .07 & -.13 & $-.25 * *$ & .09 & -.03 & $.24^{* *}$ & $.25^{* * *}$ \\
\hline Substance use & -.01 & .09 & .14 & $.20 *$ & -.14 & $-.23 * *$ & -.13 & $-.19 *$ & -.09 & $-.20 *$ & $.17^{*}$ & $.18^{*}$ \\
\hline
\end{tabular}

Note: Analyses were controlled for caregiver's age, sex, education, partnership status, chronic health problems, and for time since care recipient's first diagnosis and illness severity. $N(\mathrm{~T} 1)=147 ; N(\mathrm{~T} 2)=121, * p<.05 ; * * p<.01$ 
Associations between care specific coping and positive affect

Baseline and follow-up levels of positive affect had significant, positive associations with active coping, planning, positive reframing, acceptance and emotional support: participants accepting the stressful situation, using active coping or planning to deal with it or reframe it in a more positive way and seek emotional support from others showed a higher positive affect at baseline and at follow-up. Religion was positively associated with baseline levels of positive affect. Follow-up levels of positive affect were positively associated with baseline levels of instrumental support, and negatively associated with behavioral disengagement, self-blame, denial and substance-use (Table 4).

Associations between care specific coping and negative affect

Baseline and follow-up levels of negative affect were positively associated with baseline levels of self-blame, venting and substance use and negatively associated with positive reframing and acceptance: participants blaming themselves, venting or using substance, or those using less acceptance and positive reframing showed a higher negative affect than participants who did not engage in these coping strategies. Baseline levels of negative affect were also positively associated with behavioral disengagement, self-distraction and denial: participants using denial, behavioral disengagement or selfdistraction showed higher levels of negative affect (Table 4).

\section{Summary of Results}

The results from the correlational analyses showed meaningful associations between personality constructs, care-specific coping, and indicators of subjective wellbeing. Overall, these associations indicated that two of the three selected personality 
constructs (i.e., goal adjustment capacities and optimism) have significant associations with a number of care-specific coping behaviors. Additionally, all the indicators of subjective well-being were associated with several care-specific coping behaviors. This points to the possibility that some coping strategies may have a mediating influence on the associations between personality constructs and indicators of subjective well-being.

\section{Personality Constructs}

This section will be presented in four parts. The first part describes the correlations between the selected personality constructs (goal adjustment capacities, dispositional optimism and unmitigated communion). Parts two, three and four, present the cross-sectional and longitudinal associations between each of the selected personality construct, caregiver burden, and subjective well-being outcomes (depressive symptoms, purpose in life, satisfaction with life, positive and negative affect), as well as the mediation effect of participants' care specific coping behaviors. All analyses were controlled for the previously described covariates.

\section{Correlations between personality constructs}

The correlations between the selected personality constructs (Table 5) showed significant associations between dispositional optimism and both goal disengagement capacities and goal reengagement capacities. In particular, optimists reported better goal adjustment capacities than pessimists. The two goal adjustment capacities were also significantly associated with each other. Participants with high levels of goal disengagement capacities reported higher goal reengagement capacities as well. There was no significant association between unmitigated communion and any of the other selected personality constructs. 
Table 5

Correlations between personality constructs (goal disengagement, goal reengagement, optimism and unmitigated communion)

\begin{tabular}{ccccc}
\hline & $\begin{array}{c}\text { Goal } \\
\text { disengagement }\end{array}$ & $\begin{array}{c}\text { Goal } \\
\text { reengagement }\end{array}$ & Optimism & $\begin{array}{c}\text { Unmitigated } \\
\text { communion }\end{array}$ \\
\hline $\begin{array}{c}\text { Goal } \\
\text { disengagement } \\
\begin{array}{c}\text { Goal } \\
\text { reengagement }\end{array}\end{array}$ & 1 & & & \\
$\begin{array}{c}\text { Optimism } \\
\text { Unmitigated } \\
\text { communion }\end{array}$ & $\mathbf{. 1 9 *}$ & 1 & 1 & \\
\hline
\end{tabular}

Note: $N=147 . * p<.05 ; * * p<.01$ 
The associations between selected personality constructs, caregiver burden, and subjective well-being outcomes

\section{Goal Adjustment Capacities}

To examine whether goal adjustment capacities are associated with participants' well-being, two sets of cross-sectional and longitudinal regression analyses were performed. In these analyses baseline and follow-up levels (controlling for baseline levels) of caregiver burden, depressive symptoms, purpose in life, satisfaction with life, positive and negative affect were predicted, by participants' baseline levels of goal disengagement and goal reengagement capacities. Subsequently caregiver burden was examined in order to determine if it could explain the obtained effects on participants' general subjective well-being, by repeating the analyses for predicting indicators of subjective well-being, and additionally including baseline levels of caregiver burden into these regression analyses. Next, the interaction terms between caregiver burden and each of the goal adjustment capacities were included separately into these analyses to examine whether goal adjustment capacities can moderate the associations between caregiver burden and participants' general subjective well-being. Finally, the mediation effect that care specific coping behaviors may have on the associations between goal adjustment capacities and indicators of subjective well-being was explored by calculating Sobel tests. All analyses statistically controlled for the previously described covariates, and the predictor variables were centered prior to conducting the analyses.

\section{Cross-sectional analyses}

The results from the cross-sectional analyses are presented in Table 6 , and reveal that goal disengagement and goal reengagement capacities were significantly associated 
Table 6

Cross-sectional results from hierarchical regression analyses predicting baseline levels of depressive symptoms, purpose in life, satisfaction with life, positive and negative affect, and caregiver burden, by participants' goal adjustment capacities (Model 1) and by the interactions between caregiver burden and goal adjustment capacities (Model 2)

\begin{tabular}{|c|c|c|c|c|c|c|c|c|c|c|c|c|}
\hline & \multicolumn{2}{|c|}{$\begin{array}{l}\text { Depressive } \\
\text { symptoms }\end{array}$} & \multicolumn{2}{|c|}{$\begin{array}{l}\text { Purpose } \\
\text { in life }\end{array}$} & \multicolumn{2}{|c|}{$\begin{array}{c}\text { Satisfaction } \\
\text { with life }\end{array}$} & \multicolumn{2}{|c|}{$\begin{array}{c}\text { Positive } \\
\text { affect }\end{array}$} & \multicolumn{2}{|c|}{$\begin{array}{l}\text { Negative } \\
\text { affect }\end{array}$} & \multicolumn{2}{|c|}{$\begin{array}{l}\text { Caregiver } \\
\text { burden }\end{array}$} \\
\hline & $\mathrm{R}^{2}$ & $\beta$ & $\mathrm{R}^{2}$ & $\beta$ & $\mathrm{R}^{2}$ & $\beta$ & $\mathrm{R}^{2}$ & $\beta$ & $\mathrm{R}^{2}$ & $\beta$ & $\mathrm{R}^{2}$ & $\beta$ \\
\hline \multicolumn{13}{|l|}{ Model 1} \\
\hline Goal dis' (GD) & $.03 *$ & $-.18 *$ & 0 & .02 & $.03 *$ & $.20 *$ & .01 & -.12 & $.06 * *$ & $-.26 * *$ & $.03 *$ & $-.20 *$ \\
\hline Goal re' (GR) & 0 & -.04 & $.05 * *$ & $.26 * *$ & 0 & .06 & $.06 * *$ & $.26 * *$ & 0 & -.03 & $.03 *$ & $.20 *$ \\
\hline \multicolumn{13}{|l|}{ Model 2} \\
\hline Burden & $.07 * *$ & $.30 * *$ & $.05 * *$ & $-.24 * *$ & $.13 * *$ & $-.40 * *$ & .01 & -.13 & $.07 * *$ & $.29 * *$ & & \\
\hline Goal dis' (GD) & .01 & -.13 & 0 & -.03 & .1 & .12 & .02 & -.15 & $.03 * *$ & $-.20 * *$ & & \\
\hline Goal re' (GR) & .01 & -.10 & $.07 * *$ & $.30 * *$ & .01 & .13 & $.06 * *$ & $.29 * *$ & .01 & -.09 & & \\
\hline \multicolumn{13}{|l|}{ Interaction } \\
\hline Burden X GD & 0 & -.04 & 0 & .06 & $.03 *$ & $.17 *$ & 0 & -.01 & 0 & .04 & & \\
\hline Burden X GR & $.04 * *$ & $-.21 * *$ & .01 & .12 & 0 & .05 & .02 & .14 & 0 & -.06 & & \\
\hline
\end{tabular}

Note: Analyses were controlled for caregiver's age, sex, education, partnership status, and chronic health problems, and time since care recipient's first diagnosis and illness severity. $\mathrm{R}^{2} \mathrm{~s}$ for predictors represent unique amount of variance explained in the outcomes.

$N=147, * p<.05 ; * * p<.01$ 
with indicators of subjective well-being (Model 1). More specifically, participants with high baseline levels of goal disengagement capacities experienced lower levels of caregiver burden, $F(1,137)=5.32, p=.02$, lower levels of depressive symptoms, $F(1,137)=4.97, p=.03$, lower levels of negative affect, $F(1,137)=9.35, p<.01$, and higher levels of satisfaction with life, $F(1,137)=5.14, p=.03$, compared with participants with low levels of goal disengagement capacities. In addition, high, as compared to low, levels of goal reengagement capacities were associated with higher levels of purpose in life, $F(1,137)=8.42, p<.01$, and positive affect $F(1,137)=8.92$, $p<.01$. Interestingly, however, the results also showed that participants who had an easier time reengaging in new goals experienced greater caregiver burden than their counterparts with poor goal reengagement capacities, $F(1,137)=5.18, p=.02$. Effects were obtained above and beyond the covariates.

The subsequent inclusion of baseline levels of caregiver burden into the models (Table 6, Model 2) demonstrated that caregiver burden was significantly associated with higher baseline levels of depressive symptoms, $F(1,136)=13.93, p<.01$ and negative affect $F(1,136)=12.94, p<.01$, and with lower baseline levels of purpose in life, $F(1,136)=7.61, p<.01$, and satisfaction with life $F(1,136)=24.70, p<.01$. Moreover caregiver burden rendered the main effect of goal disengagement on depressive symptoms and on satisfaction with life non-significant, and exerted a significant indirect effect on the association between goal disengagement and depressive symptoms and on the association between goal disengagement and satisfaction with life (Sobel test: depressive symptoms, $Z=-1.96, \mathrm{p}=.05$, satisfaction with life, $\mathrm{Z}=2.13, \mathrm{p}=.03$ ). These findings imply that participants who were able to disengage from unattainable goals also 
experienced relatively low levels of caregiver burden, which explained their lower levels of depressive symptoms and higher levels of satisfaction with life.

However, caregiver burden did not explain the association between adaptive goal disengagement tendencies and higher levels of negative affect. Additionally, caregiver burden did not explain the association between adaptive goal reengagement tendencies and higher levels of purpose in life or positive affect. Finally, the analyses demonstrated two significant interaction effects: the interaction between caregiver burden and goal disengagement capacities in predicting participants' satisfaction with life, $F(1,135)=$ $5.43, p=.02$, and the interaction between caregiver burden and goal reengagement capacities in predicting participants' depressive symptoms, $F(1,135)=8.21, p<.01$. Caregiver burden did not interact with goal disengagement or goal reengagement for predicting levels of purpose in life, positive affect, or negative affect; it also did not interact with goal disengagement for predicting depressive symptoms or with goal reengagement for predicting satisfaction with life.

The significant interaction effects are illustrated in Figure 1 and Figure 2 by plotting the associations between caregiver burden and the well-being outcomes depressive symptomatology and satisfaction with life, separately for participants with low versus high levels of goal adjustment capacities (one standard deviation above and below the sample means, see Aiken \& West, 1991). Figure 1 demonstrates the association between burden and depressive symptomatology separately for participants with low versus high levels of goal reengagement capacities. The obtained pattern of results indicated that elevated baseline levels of depressive symptoms were obtained particularly among participants who experienced high levels of caregiver burden and had difficulty 
with goal reengagement. By contrast, participants who experienced high levels of caregiver burden and had an easier time reengaging in new goals experienced significantly lower levels of depressive symptoms, comparable to participants who generally reported low levels of caregiver burden. Consistent with this interpretation, analyses of the simples slopes demonstrated that caregiver burden was significantly associated with high levels of depressive symptoms among participants who had difficulty with goal reengagement, $\beta=.56, p<.01$, but not among participants with high levels of goal reengagement capacities, $\beta=.10, p=.38$. Moreover, goal reengagement capacities were significantly associated with lower levels of depressive symptoms among participants with high caregiver burden, $\beta=-.39, p<.01$, but not among their counterparts with low levels of caregiver burden, $\beta=.08, p=.40$.

Figure 2 demonstrates the association between burden and satisfaction with life separately for participants with low versus high levels of goal disengagement capacities. The obtained pattern of results indicated that lower baseline levels of satisfaction with life were obtained particularly among participants who experienced high levels of caregiver burden and had difficulty with goal disengagement. By contrast, participants who experienced high levels of caregiver burden and had an easier time disengaging from unattainable goals experienced significantly higher levels of satisfaction with life, comparable to participants who generally reported low levels of caregiver burden. Consistent with this interpretation, analyses of the simples slopes demonstrated that caregiver burden was significantly associated with low levels of satisfaction with life among participants who had difficulty with goal disengagement, $\beta=-.59, p<.01$, but not among participants with high levels of goal disengagement capacities, $\beta=-.19, p=.14$. 


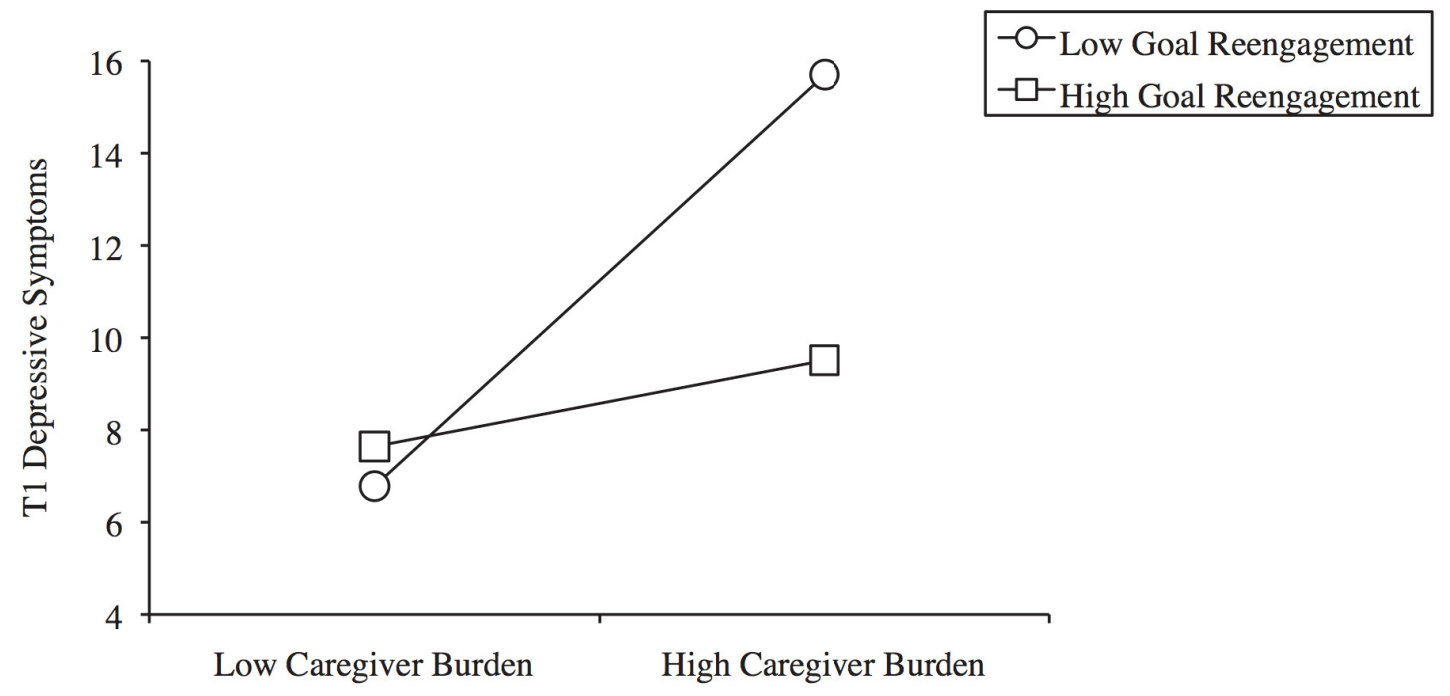

Figure 1. Associations between baseline levels of caregiver burden and depressive symptoms, separately for participants with high versus low baseline levels of goal reengagement capacities 


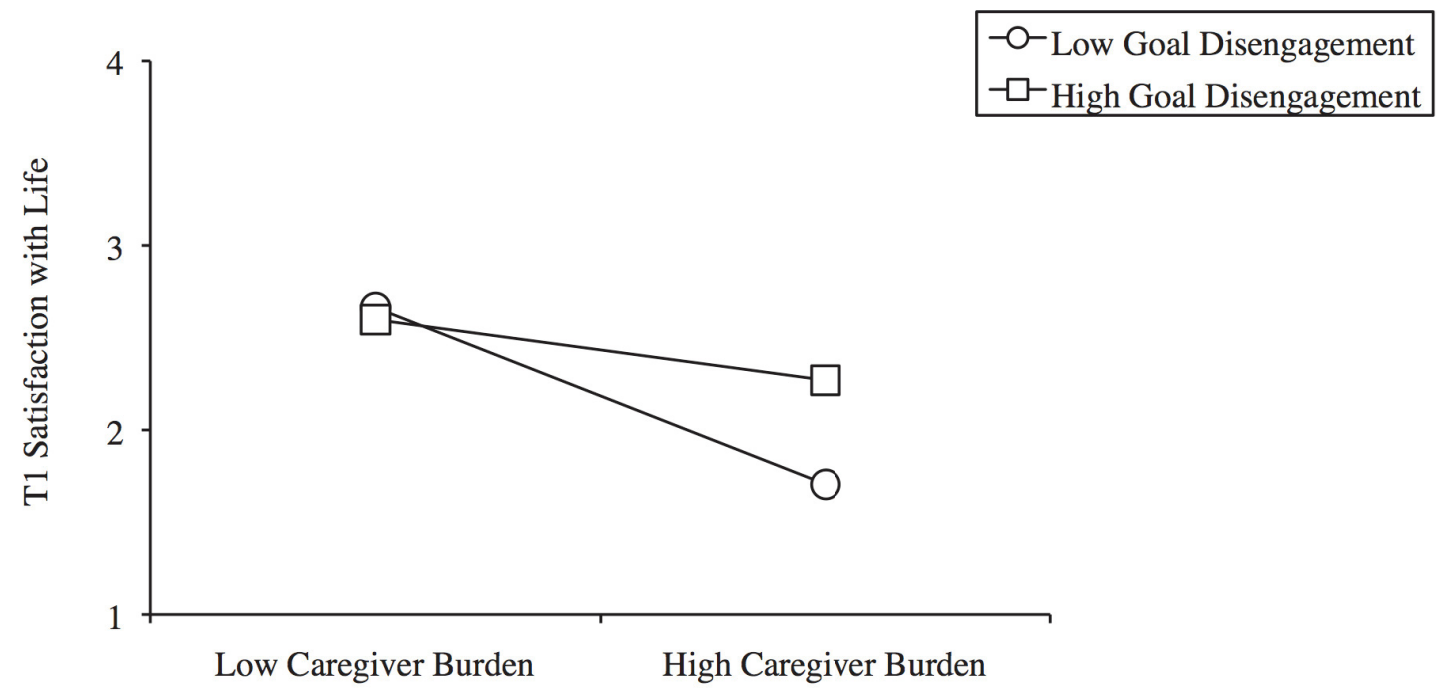

Figure 2. Associations between baseline levels of caregiver burden and satisfaction with life, separately for participants with high versus low baseline levels of goal disengagement capacities 
Moreover, goal disengagement capacities were significantly associated with lower levels of satisfaction with life among participants with high caregiver burden, $\beta=.28 p=.03$ but not among their counterparts with low levels of caregiver burden, $\beta=.13, p=.19$.

\section{Longitudinal analyses}

The results from the longitudinal analyses are presented in Table 7, and show that baseline levels of the outcomes were significantly associated with follow-up levels of participants' caregiver burden, depressive symptoms, purpose in life, satisfaction with life, positive affect and negative affect, $F \mathrm{~s}(1,110)>51.70, \beta \mathrm{s}>.56, R^{2} \mathrm{~s}>.27, p \mathrm{~s}<.01$. However, the main effects of baseline levels of goal disengagement or goal reengagement did not significantly predict changes in the outcome variables (Table 7, Model 1).

The subsequent inclusion of caregiver burden into the models showed that baseline levels of caregiver burden predicted larger increases of depressive symptoms over time, $F(1,109)=4.29, p=.04$, but was not associated with changes in purpose in life, satisfaction with life, positive affect, or negative affect (Table 7, Model 2). In addition, the analyses demonstrated significant interaction effects, but only for the interaction between caregiver burden and goal disengagement capacities on changes in depressive symptoms, $F(1,108)=4.29, p=.04$, and on changes in negative affect, $F(1,108)=4.78$, $p=.03$ (Table 7, Model 2). The significant interaction effects are illustrated in Figures 3 and Figure 4, employing the previously described procedures. The interaction effect between caregiver burden and goal disengagement capacity on changes in depressive symptoms (Figure 3) suggests that increases in depressive symptoms were observed particularly among participants who experienced high levels of caregiver burden at baseline and had poor goal disengagement capacities. By contrast, depressive symptom 
Table 7

Longitudinal results from hierarchical regression analyses predicting baseline levels of depressive symptoms, purpose in life, satisfaction with life, positive and negative affect, and caregiver burden, by participants' goal adjustment capacities (Model 1) and by the interactions between caregiver burden and goal adjustment capacities (Model 2)

\begin{tabular}{|c|c|c|c|c|c|c|c|c|c|c|c|c|}
\hline & \multicolumn{2}{|c|}{$\begin{array}{c}\text { T2 Depressive } \\
\text { symptoms }\end{array}$} & \multicolumn{2}{|c|}{$\begin{array}{c}\text { T2 Purpose in } \\
\text { life }\end{array}$} & \multicolumn{2}{|c|}{$\begin{array}{l}\text { T2 Satisfaction } \\
\text { with life }\end{array}$} & \multicolumn{2}{|c|}{$\begin{array}{c}\text { T2 Positive } \\
\text { affect }\end{array}$} & \multicolumn{2}{|c|}{$\begin{array}{l}\text { T2 Negative } \\
\text { affect }\end{array}$} & \multicolumn{2}{|c|}{$\begin{array}{c}\text { T2 Caregiver } \\
\text { burden }\end{array}$} \\
\hline & $\mathrm{R}^{2}$ & $\beta$ & $\mathrm{R}^{2}$ & $\beta$ & $\mathrm{R}^{2}$ & $\beta$ & $\mathrm{R}^{2}$ & $\beta$ & $\mathrm{R}^{2}$ & $\beta$ & $\mathrm{R}^{2}$ & $\beta$ \\
\hline \multicolumn{13}{|l|}{ Model 1} \\
\hline T1 Outcomes & $.30 * *$ & $.58 * *$ & $.27 * *$ & $.56 * *$ & $.47 * *$ & $.75 * *$ & $.45 * *$ & $.75 * *$ & $.36 * *$ & $.63 * *$ & $.51 * *$ & $.77 * *$ \\
\hline Goal re' (GR) & .01 & .12 & .01 & .11 & .01 & -.13 & 0 & .02 & 0 & .06 & & \\
\hline \multicolumn{13}{|l|}{ Model 2} \\
\hline Tl Outcomes & $.24 * *$ & $.54 * *$ & $.24 * *$ & $.55^{* *}$ & $.34 * *$ & $.71^{* *}$ & $.42 * *$ & $.73 * *$ & $.30 * *$ & $.60 * *$ & & \\
\hline Goal re' (GR) & .01 & .09 & .01 & .12 & .01 & -.1 & 0 & .05 & 0 & .03 & & \\
\hline \multicolumn{13}{|l|}{ Interaction } \\
\hline Burden X GD & $.02 *$ & $-.14 *$ & .01 & -.13 & 0 & -.01 & 0 & 0 & $.02 *$ & $-.16 *$ & & \\
\hline Burden X GR & 0 & .03 & 0 & -.06 & 0 & -.05 & 0 & -.01 & 0 & -.07 & & \\
\hline
\end{tabular}

Note: Analyses were controlled for caregiver's age, sex, education, partnership status, and chronic health problems, and time since care recipient's first diagnosis and illness severity. $\mathrm{R}^{2} \mathrm{~s}$ for predictors represent unique amount of variance explained in the outcomes.

$N=121, * p<.05 ; * * p<.01$ 


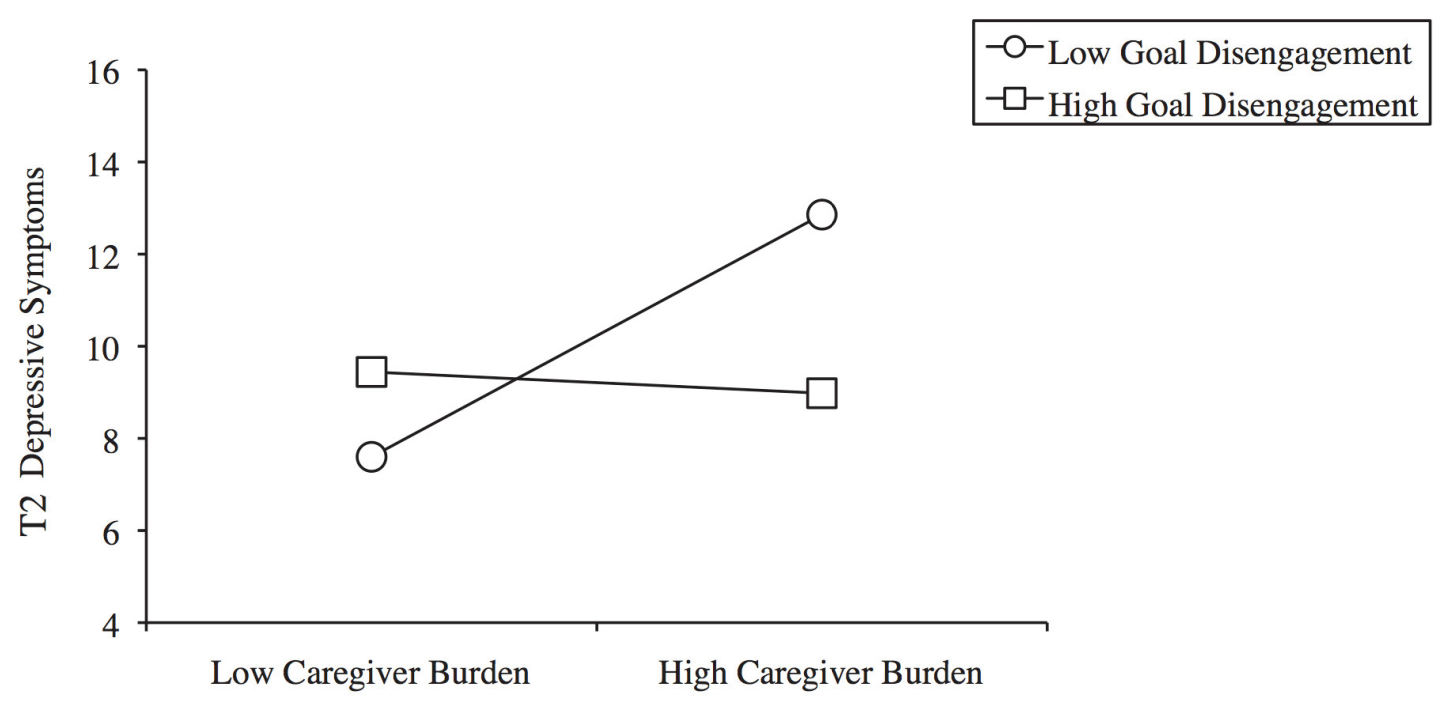

Figure 3. Associations between baseline levels of caregiver burden and changes in depressive symptoms, separately for participants with high versus low baseline levels of goal disengagement capacities 
were fairly stable over time among less burdened caregivers, and highly burdened caregivers who had an easier time disengaging from unattainable goals. Consistent with this interpretation, analyses of the simples slopes demonstrated that caregiver burden was significantly associated with larger increases in depressive symptoms among participants who reported low, $\beta=.29, p<.01$, but not high levels of goal disengagement capacities, $\beta=-.03, p=.78$. Moreover, adaptive goal disengagement capacities were marginally associated with fewer increases in depressive symptoms among participants with relatively high, $\beta=-.21, p=.06$, but not low, levels of caregiver burden, $\beta=.10, p=.33$.

The interaction effect between goal disengagement and burden on changes in negative affect (Figure 4) was similar to the effect of the same interaction on depressive symptoms, and suggests that increases in negative affect was obtained particularly among participants who experienced high levels of caregiver burden at baseline and poor goal disengagement capacities. By contrast, participants who experienced high levels of caregiver burden and had an easier time disengaging from unattainable goals experienced significantly fewer increases in negative affect, comparable to their counterparts who reported low baseline levels of caregiver burden. Analyses of the simples slopes demonstrated that caregiver burden was significantly associated with larger increases in negative affect among participants who had difficulty with goal disengagement, $\beta=.27$, $p<.01$, but not among participants with high levels of goal disengagement capacities, $\beta=$ $-.09, p=.49$. Moreover, adaptive goal disengagement capacities were marginally associated with fewer increases in negative affect among participants with high levels of caregiver burden, $\beta=-.21, p=.08$, but not among their counterparts with low levels of caregiver burden, $\beta=.14, p=.19$. 


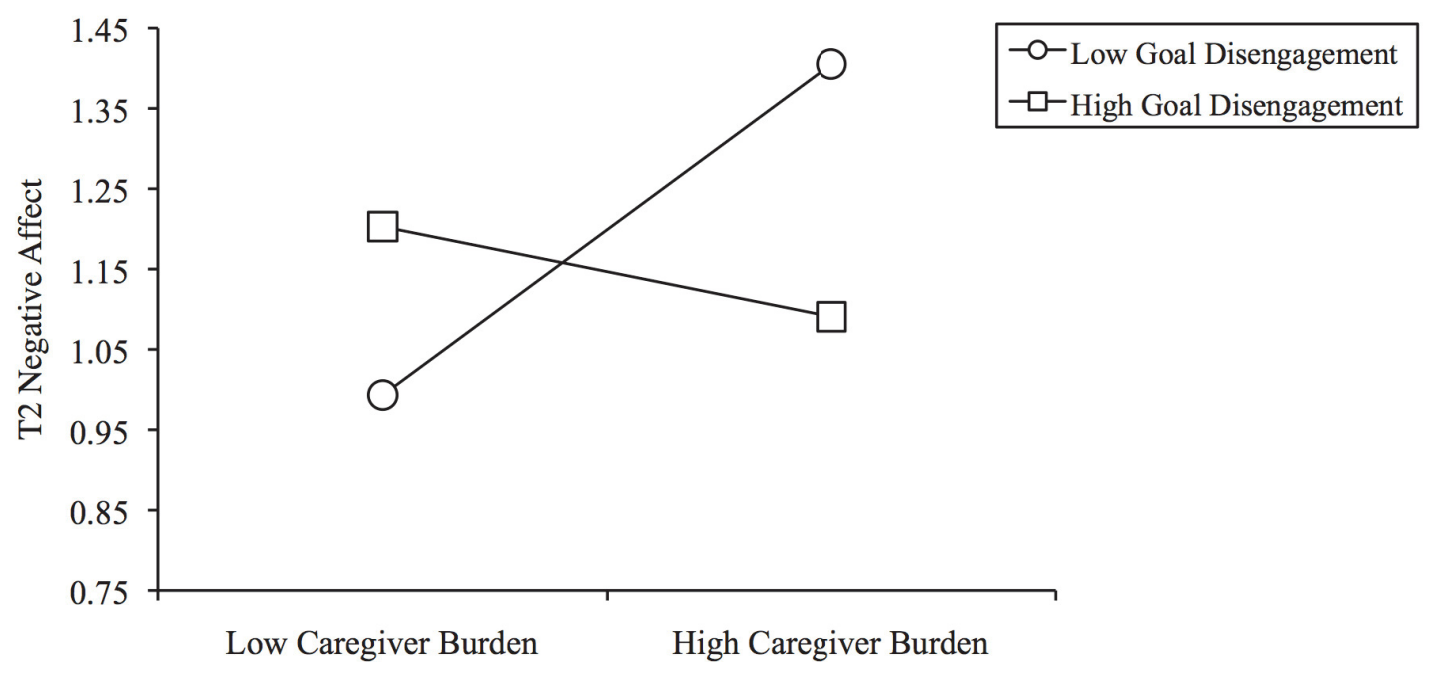

Figure 4. Associations between baseline levels of caregiver burden and changes in negative affect, separately for participants with high versus low baseline levels of goal disengagement capacities 


\section{The Mediating Role of Coping}

To examine whether the use of care-specific coping statistically explained the observed effects of goal adjustment capacities on participants'subjective well-being, potential mediators were first identified by considering those coping strategies that were associated with goal disengagement (i.e., self-blame and substance use) or goal reengagement capacities (i.e., active coping, planning, instrumental support, positive reframing, humor, religion, self-distraction, venting, and substance use, see Table 3), and that were significantly associated with the respective outcomes. In this regard, Table 4 presents the associations between baseline levels of coping, and baseline and follow-up levels of caregiver burden, depressive symptoms, purpose in life, satisfaction with life, positive affect and negative affect. The results indicate that self-blame and substance use were significantly associated with higher caregiver burden or with lower levels of indicators of subjective well-being (depressive symptoms, purpose in life, satisfaction with life, positive affect and negative affect), and therefore qualified as potential mediators of the effects of goal disengagement on these outcomes. In addition, active coping, planning, instrumental support, positive reframing, and religion were associated with higher baseline levels of purpose and positive affect, or lower baseline levels of depressive symptoms, and thus were identified as coping strategies that could explain the effects of goal reengagement on these outcomes. Additionally, positive reframing and self-distraction were significantly associated with negative affect, therefore could mediate the effect of goal reengagement on negative affect. Finally, venting, humor, and selfdistraction were significantly associated with greater caregiver burden, and therefore could mediate the effect of goal reengagement on higher levels of caregiver burden. 
The next step attempted to statistically explain the cross-sectional effects of goal adjustment capacities on baseline levels of caregiver burden, depressive symptoms, satisfaction with life, and negative affect (Table 6, Models 1) by the identified coping strategies. The results showed that the inclusion of self-blame and substance use rendered the cross-sectional effects of goal disengagement on baseline levels of caregiver burden, $F(1,135)=2.82, \beta=-.14, p=.10, R^{2}=.02$, and depressive symptoms, $F(1,135)=2.67, \beta$ $=-.14, p=.10, R^{2}=.01$ non-significant (explaining $30.46 \%$ and $46.43 \%$ of the effects on caregiver burden, depressive symptoms, respectively). However, only self-blame was independently associated with levels of caregiver burden, $F(1,136)=9.68, \beta=.26, p<$ $.01, R^{2}=.06$, and depressive symptoms, $F(1,136)=4.31, \beta=.17, p=.04, R^{2}=.02$, and Sobel tests demonstrated that goal disengagement exerted a significant indirect effect through self-blame on caregiver burden, $Z=-2.02, p=.04$, and a marginally significant indirect effect on depressive symptoms, $Z=-1.64, p=.10$. These findings suggest that participants who were better able to disengage from unattainable goals also blamed themselves relatively infrequently for caregiving problems, which explained their lower levels of caregiver burden and depressive symptoms.

Exploring the potential mediation of coping behaviors on the association between goal disengagement and negative affect revealed that only self blame was significantly associated with negative affect, $\mathrm{F}(1,136)=26.01, \beta=.40, p<.01, \mathrm{R}^{2}=13$, and a Sobel test confirmed that goal disengagement exerted a significant indirect effect through selfblame on negative affect, $\mathrm{Z}=-2.36, p<.01$. Similar to the previous findings, this finding suggests that participants who were better able to disengage from unattainable goals also blamed themselves less frequently for their caregiving problems, which explained their 
lower levels of negative affect.

Self-blame was the only coping behavior found to have a potential mediation effect on the association between goal disengagement and satisfaction with life, $\mathrm{F}(1,136)$ $=5.07, \beta=-.19, p=.03, \mathrm{R}^{2}=03$. However, a Sobel test showed only a marginal indirect effect of self-blame $(\mathrm{Z}=1.71, p=.09)$ on the association between goal disengagement and satisfaction with life.

In addition, the effect of goal reengagement on baseline levels of purpose in life (Table 6, Model 1) became non-significant, $F(1,132)=1.15, \beta=.10, p=.29, R^{2}=.01$, if the identified five coping strategies (active coping, planning, instrumental support, positive reframing, and religion), were included into the model, explaining $88.68 \%$ of the effect. However, only positive reframing exerted a significant and independent effect on purpose in life, $F(1,136)=20.48, \beta=.36, p<.01, R^{2}=.11$, and a Sobel test confirmed a significant indirect effects of goal reengagement capacities on greater purpose in life through high levels of positive reframing, $Z=-2.50, p=.01$. These findings indicate that caregivers who were able to identify and pursue new goals evaluated the stressful circumstances in a more positive light, which explained their higher levels of purpose in life.

Exploring the potential mediation of coping behaviors on the association between goal reengagement and positive affect revealed that of the included four potential mediators (active coping, planning, positive reframing and religion), only positive reframing showed a significant independent association with positive affect, $\mathrm{F}(1,136)=$ $1.09, \beta=.18 p<.01$. A subsequently conducted Sobel test confirmed that goal reengagement exerted a significant indirect effect through positive reframing on positive 
affect, $Z=2.34, p=.02$, suggesting that caregivers who were able to identify and pursue new goals, evaluated stressful situations in a more positive light, which explained their high levels of positive affect.

Finally, the effect of goal reengagement on elevated baseline levels of caregiver burden (Table 6, Model 1) was rendered non-significant, $F(1,134)=1.22, \beta=.10, p=$ $.27, R^{2}=.01$, if the identified three coping strategies (venting, self-distraction, or humor) were included into the model (explaining $77.42 \%$ of the effect) into the model. However, none of the three variables showed an independent effect on caregiver burden, although Sobel tests suggested marginally significant indirect effects of goal reengagement capacities on greater caregiver burden through venting, $Z=1.83, p=.07$, and selfdistraction, $Z=1.75, p=.08$, but not through humor. It is important to note that venting and self-distraction were significantly correlated with each other, $r=.32, p<.01$, and a compound variable (i.e., the average of venting and self-distraction) exerted an independent effect on caregiver burden, $F(1,135)=5.53, \beta=.20, p<.05, R^{2}=.03$, and significantly mediated the association between goal reengagement and greater caregiver burden, $Z=2.29, p=.02$. These findings indicate that the higher levels of burden observed among caregivers who engaged in other new goals, could be explained by more frequent venting and self-distraction among these participants.

In a second set of analyses, it was examined whether the cross-sectional buffering effect of goal reengagement on the association between caregiver burden and depressive symptoms (Table 6, Model 2) could be statistically explained by the identified coping strategies. To this end, the analysis for predicting levels of depressive symptoms was repeated, and included the five identified coping strategies and their interactions with 
caregiver burden and goal reengagement (for statistically controlling moderator effects, see Yzerbyt, Muller, \& Judd, 2004). The results of the analysis showed that the interaction effect between caregiver burden and goal reengagement on depressive symptoms was no longer significant, $F(1,120)=2.62, \beta=-.15, p=.11, R^{2}=.01$, if the variance associated with coping was accounted for, which explained $70.00 \%$ of the interaction effect. In this analysis, only positive reframing showed an independent effect on depressive symptoms, $F(1,120)=6.50, \beta=-.22, p=.01, R^{2}=.03$, suggesting that the adaptive buffering effect of goal reengagement on the association between caregiver burden and depressive symptoms was related to the more frequent use of positive reframing among participants with high levels of goal reengagement capacities.

Next, it was examined whether the cross-sectional buffering effect of goal disengagement on the association between caregiver burden and satisfaction with life (Table 6, Model 2) could be statistically explained by self-blame (the only potential mediator). The results of the analysis showed that the interaction effect between caregiver burden and goal disengagement on satisfaction with life remained significant after selfblame was included in the analysis, $F(1,132)=9.70, \beta=.24, p<.01, R^{2}=.05$. This suggests that self-blame has no mediating effect on the interaction between caregiver burden and goal disengagement and their association with satisfaction with life.

Finally, it was examined whether self-blame and substance use would mediate the significant longitudinal interaction effect between caregiver burden and goal disengagement capacities on changes in depressive symptoms and changes in negative affect. This was accomplished by incorporating the main effects of these coping strategies and their interactions with caregiver burden and goal disengagement into the 
previously reported analysis (Table 7, Model 2). The inclusion of the variance associated with these coping strategies in relation to changes in depressive symptoms rendered the interaction effect between caregiver burden and goal disengagement capacities on changes in depressive symptoms non-significant, $F(1,102)=2.82, \beta=-.12, p=.10, R^{2}=$ .01 , explaining $38.89 \%$ of the interaction effect. While neither self-blame nor substance use exerted significant main effects in this analyses, the reduction of the interaction between caregiver burden and goal disengagement was associated with a significant interaction effect between caregiver burden and substance use on changes in depressive symptoms, $F(1,102)=7.63, \beta=.19, p<.01, R^{2}=.03$, and remained significant if this interaction was not included in the analysis. This interaction effect showed that highly burdened participants who frequently used substances experienced the largest increases in depressive symptoms over time. Although these findings only suggest partial mediation, they are consistent with the idea that among highly burdened caregivers, being able to disengage from unattainable goals is protective against depressive symptoms, partly because it reduces the likelihood of coping through substance use.

Analyzing the potential mediation effects that self-blame and substance use may have on the association between the interaction caregiver burden and disengagement and changes in negative affect, rendered the interaction effect of caregiver burden and disengagement capacities non-significant, $F(1,102)=2.75, \beta=-.13, p=.10, R^{2}=.01$. Only self-blame had a significant unique effect in this analysis $(\beta=.21, p=.02)$, suggesting that burdened participants who blamed themselves experienced the largest increases of negative affect over time. Like with depression, it points to the likelihood that ability to disengage from unattainable goals is protective against negative affect, 
partly because of using less self-blame.

\section{Dispositional Optimism}

To examine whether optimism is associated with participants' well-being, two sets of cross-sectional and longitudinal regression analyses were performed. The results are presented in Table 8 and Table 9. In these analyses, baseline and follow-up levels (controlling for baseline levels) of caregiver burden, depressive symptoms, purpose in life, satisfaction with life, positive, and negative affect, were predicted by participants' baseline levels of optimism. Subsequently caregiver burden was examined in order to determine whether it could explain the obtained effects on participants' general subjective well-being. To this end, the analyses for predicting indicators of subjective well-being were repeated, and baseline levels of caregiver burden were additionally included into these regression analyses. Next, the interaction terms between caregiver burden and optimism were included into these analyses to examine whether optimism can moderate the association between caregiver burden and participants' general subjective well-being. Finally, the mediation effects that care specific coping behaviors may have on the associations between dispositional optimism and indicators of subjective wellbeing were explored. All analyses statistically controlled for the previously described covariates, and the predictor variables were centered prior to conducting the analyses.

\section{Cross-sectional analyses}

The results from the cross-sectional analyses revealed that optimism had a significant positive associations with baseline levels of purpose in life, satisfaction with life and positive affect, and significant negative associations with depressive symptoms, negative affect and burden (Table 8, Model 1). More specifically, optimists (as compared to pessimists) experienced higher levels of purpose in life, $F(1,138)=61.55, p<.01$, 
satisfaction with life $F(1,138)=70.65, p<.01$, and positive affect $F(1,138)=29.07, p<$ .01 , and lower levels of depressive symptoms, $F(1,138)=40.76, p<01$, negative affect $F(1,138)=48.45, p<.01$, and burden $F(1,138)=3.73, p<.05$.

The subsequent inclusion of baseline levels of caregiver burden into the model (Table 8, Model 2) demonstrated that caregiver burden was significantly associated with higher baseline levels of depressive symptoms $F(1,137)=10.69, p<.01$, and negative affect $F(1,137)=10.30, p<.01$, and lower levels of satisfaction with life $F(1,137)=$ $21.65, p<.01$. However, the main effect of optimism on all the indicators of subjective well-being remained significant when burden was included. In addition, the analyses demonstrated no significant interaction effects between caregiver burden and optimism on any of the indicators of subjective well-being.

Although these findings suggest that burden did not fully mediate the associations between optimism and subjective well-being, Sobel tests revealed a trend of indirect effect of burden as mediator on the association between optimism and depressive symptoms $(Z=-1.67, p=.09)$, on the association of optimism with satisfaction with life $(Z=1.76, p=.08)$, and on the association between optimism and negative affect $(Z=-$ $1.66, p=.10)$. This suggests that beneficial effects of optimism on subjective well-being are only marginally mediated through caregiver burden. 
Table 8

Cross-sectional results from hierarchical regression analyses predicting baseline levels of depressive symptoms, purpose in life, satisfaction with life, positive and negative affect, and caregiver burden, by participants' optimism (Model 1) and by the interactions between caregiver burden and optimism (Model 2)

\begin{tabular}{|c|c|c|c|c|c|c|c|c|c|c|c|c|}
\hline & \multicolumn{2}{|c|}{$\begin{array}{l}\text { Depressive } \\
\text { symptoms }\end{array}$} & \multicolumn{2}{|c|}{$\begin{array}{l}\text { Purpose } \\
\text { in life }\end{array}$} & \multicolumn{2}{|c|}{$\begin{array}{c}\text { Satisfaction } \\
\text { with life }\end{array}$} & \multicolumn{2}{|c|}{$\begin{array}{c}\text { Positive } \\
\text { affect }\end{array}$} & \multicolumn{2}{|c|}{$\begin{array}{l}\text { Negative } \\
\text { affect }\end{array}$} & \multicolumn{2}{|c|}{$\begin{array}{l}\text { Caregiver } \\
\text { burden }\end{array}$} \\
\hline & $\mathrm{R}^{2}$ & $\beta$ & $\mathrm{R}^{2}$ & $\beta$ & $\mathrm{R}^{2}$ & $\beta$ & $\mathrm{R}^{2}$ & $\beta$ & $\mathrm{R}^{2}$ & $\beta$ & $\mathrm{R}^{2}$ & $\beta$ \\
\hline \multicolumn{13}{|l|}{ Model 1} \\
\hline Optimism(OPT) & $.18 * *$ & $-.44 * *$ & $.29 * *$ & $.55 * *$ & $.30 * *$ & $.56 * *$ & $.16 * *$ & $.41 * *$ & $.23 * *$ & $-.49 * *$ & $.02 *$ & $-.15 *$ \\
\hline \multicolumn{13}{|l|}{ Model 2} \\
\hline Burden & $.05 * *$ & $.23 * *$ & .01 & -.10 & $.08 * *$ & $-.31 * *$ & 0 & 0 & $.05 * *$ & $.23 * *$ & & \\
\hline Optimism & $.15^{* * *}$ & $-.41 * *$ & $.26 * *$ & $.54 * *$ & $.25 * *$ & $.52 * *$ & $.15^{* *}$ & $.41 * *$ & $.19 * *$ & $-.46 * *$ & & \\
\hline \multicolumn{13}{|l|}{ Interactions } \\
\hline Burden X OPT & .01 & -.10 & 0 & -.07 & 0 & -.07 & .02 & -.15 & .01 & -.10 & & \\
\hline
\end{tabular}

Note: Analyses were controlled for caregiver's age, sex, education, partnership status, and chronic health problems, and time since care recipient's first diagnosis and illness severity. $\mathrm{R}^{2} \mathrm{~s}$ for predictors represent unique amount of variance explained in the outcomes. $N=147, * p<.05 ; * *<<.01$ 


\section{Longitudinal analyses}

The results from the longitudinal analyses are presented in Table 9 and show that baseline levels of the outcomes were significantly associated with follow-up levels of participants' caregiver burden, depressive symptoms, purpose in life, satisfaction with life, positive and negative affect, $F \mathrm{~s}(1,111)>37.30, \beta \mathrm{s}>.54, R^{2} \mathrm{~s}>.19, p \mathrm{~s}<.01$. There was no significant main effect of baseline levels of optimism on the change in indicators of subjective well-being over time (Model 1). The subsequent inclusion of baseline levels of caregiver burden into the models (Model 2) demonstrated a significant association between baseline levels of caregiver burden and increases in depressive symptoms over time, $F(1,110)=5.57, p=.02$. Finally, the analyses demonstrated a significant effect of the interaction between caregiver burden and optimism on changes in satisfaction with life, $F(1,109)=8.53, p<.01$ and on changes in positive affect over time $\mathrm{F}(1,109)=8.33, p<.01($ Model 2$)$.

The significant interaction effects are illustrated in Figure 5 and 6, employing the previously described procedures. The observed effect of the interaction between optimism and burden on follow-up levels of satisfaction with life (see Figure 5) suggests that increases in satisfaction with life over time were obtained particularly among participants who experienced low levels of caregiver burden at baseline and high level of optimism. By contrast, participants who experienced low levels of caregiver burden and low level of optimism experienced significantly fewer increases in satisfaction with life comparable to pessimist participants who reported high baseline levels of caregiver burden. Analyses of simples slopes demonstrated that caregiver burden was significantly associated with smaller increases in satisfaction with life among participants 
Table 9

Longitudinal results from hierarchical regression analyses predicting baseline levels of depressive symptoms, purpose in life, satisfaction with life, positive and negative affect, and caregiver burden, by participants' optimism (Model 1) and by the interactions between caregiver burden and optimism (Model 2)

\begin{tabular}{|c|c|c|c|c|c|c|c|c|c|c|c|c|}
\hline & \multicolumn{2}{|c|}{$\begin{array}{c}\text { T2 Depressive } \\
\text { symptoms }\end{array}$} & \multicolumn{2}{|c|}{$\begin{array}{c}\text { T2 Purpose in } \\
\text { life }\end{array}$} & \multicolumn{2}{|c|}{$\begin{array}{l}\text { T2 Satisfaction } \\
\text { with life }\end{array}$} & \multicolumn{2}{|c|}{$\begin{array}{c}\text { T2 Positive } \\
\text { affect }\end{array}$} & \multicolumn{2}{|c|}{$\begin{array}{l}\text { T2 Negative } \\
\text { affect }\end{array}$} & \multicolumn{2}{|c|}{$\begin{array}{l}\text { T2 Caregiver } \\
\text { burden }\end{array}$} \\
\hline & $\mathrm{R}^{2}$ & $\beta$ & $\mathrm{R}^{2}$ & $\beta$ & $\mathrm{R}^{2}$ & $\beta$ & $\mathrm{R}^{2}$ & $\beta$ & $\mathrm{R}^{2}$ & $\beta$ & $\mathrm{R}^{2}$ & $\beta$ \\
\hline \multicolumn{13}{|l|}{ Model 1} \\
\hline T1 Outcomes & $.22 * *$ & $.56 * *$ & $.19 * *$ & $.54 * *$ & $.31 * *$ & $.71 * *$ & $.38 * *$ & $.71 * *$ & $.32 * *$ & $.66 * *$ & $.51 * *$ & $.76 * *$ \\
\hline Optimism(OPT) & 0 & -.04 & .01 & .10 & 0 & .03 & .01 & .1 & 0 & .05 & 0 & -.02 \\
\hline \multicolumn{13}{|l|}{ Model 2} \\
\hline T1 Outcomes & $.18 * *$ & $.52 * *$ & $.19 * *$ & $.54 * *$ & $.22 * *$ & $.65^{* *}$ & $.38 * *$ & $.72 * *$ & $.26 * *$ & $.62 * *$ & & \\
\hline Burden & $.02 *$ & $.17 *$ & 0 & .03 & 0 & -.11 & .01 & -.10 & .02 & .14 & & \\
\hline Optimism & 0 & -.02 & .01 & .10 & .01 & .03 & 0 & .08 & 0 & .06 & & \\
\hline \multicolumn{13}{|l|}{ Interactions } \\
\hline Burden X OPT & .01 & .11 & .01 & -.12 & $.03 * *$ & $-.19 * *$ & $.03 * *$ & $-.18 * *$ & 0 & .01 & & \\
\hline
\end{tabular}

Note: Analyses were controlled for caregiver's age, sex, education, partnership status, and chronic health problems, and time since care recipient's first diagnosis and illness severity. $\mathrm{R}^{2} \mathrm{~s}$ for predictors represent unique amount of variance explained in the outcomes.

$N=121, * p<.05 ; * * p<.0 .1$ 


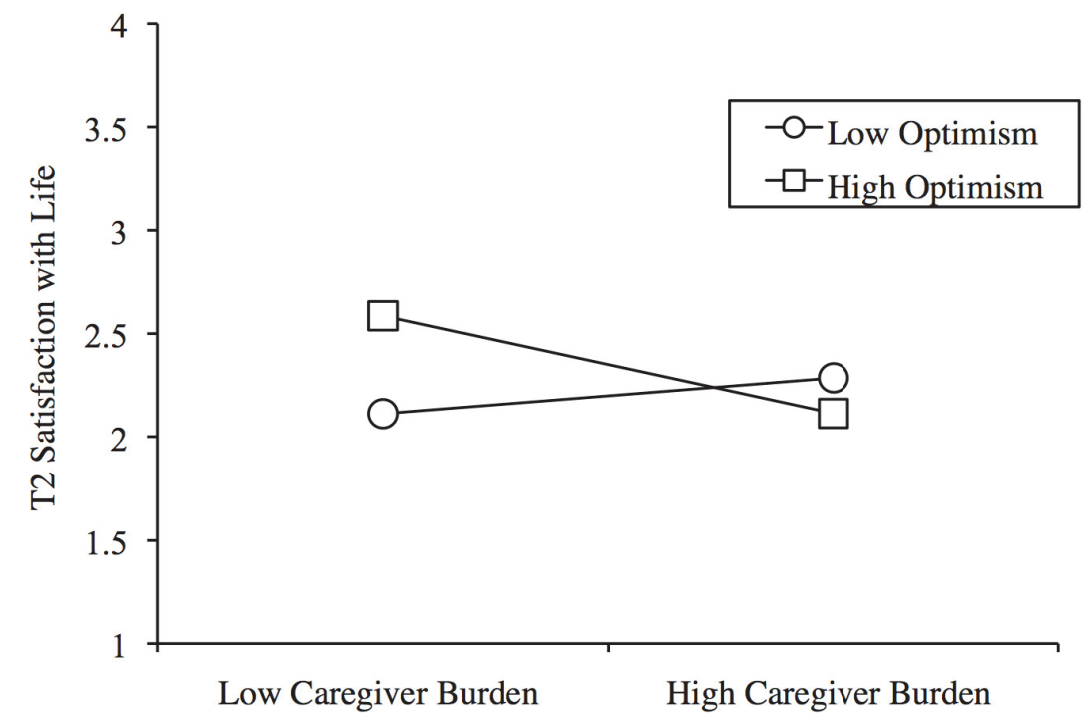

Figure 5. Associations between baseline levels of caregiver burden and changes in satisfaction with life, separately for participants with high versus low optimism 


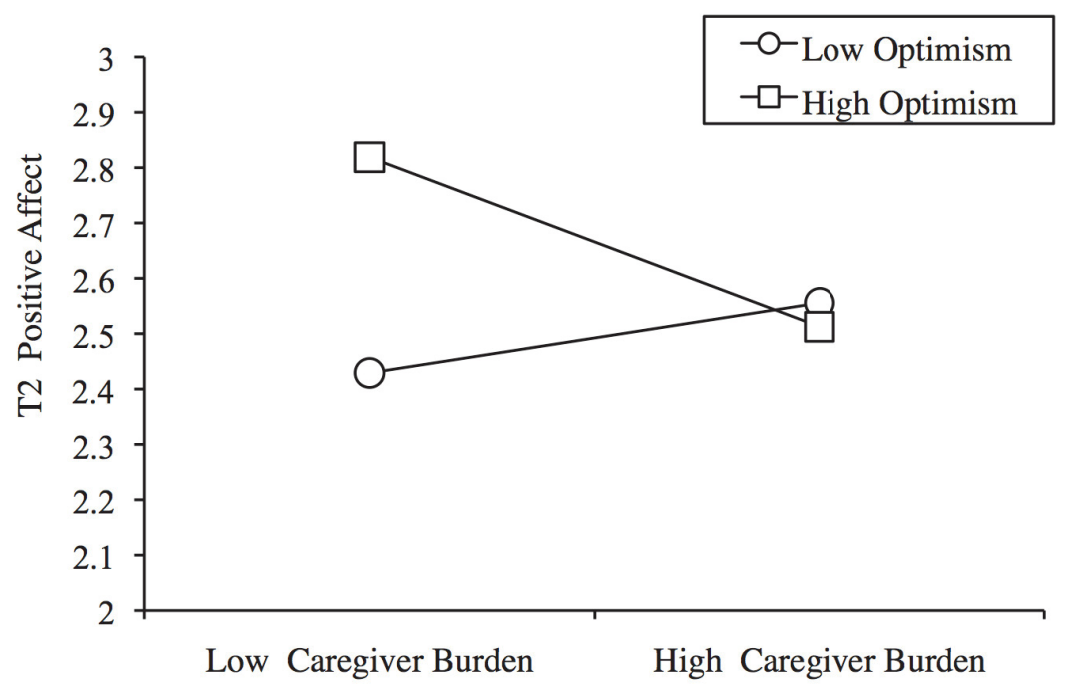

Figure 6. Associations between baseline levels of caregiver burden and change in positive affect, separately for participants with high versus low optimism 
who had high levels of optimism, $\beta=-.30 p<.01$, but not among participants with low levels of optimism, $\beta=.12, p=.26$. Moreover, optimism was significantly associated with higher increase in satisfaction with life among participants with low caregiver burden, $\beta=.30, p<.01$, but not among their counterparts with high levels of caregiver burden, $\beta=-.10, p=.25$.

A similar pattern of findings was observed for the effect of the interaction between optimism and burden on the change in positive affect over time (see Figure 6). Increases in positive affect over time were observed particularly among participants who experienced low levels of caregiver burden at baseline and high level of optimism. By contrast, participants who experienced low levels of caregiver burden and low level of optimism experienced significantly fewer increases in positive affect comparable with participants who reported high baseline levels of caregiver burden. Analyses of simples slopes demonstrated that caregiver burden had a significant association with smaller increases in positive affect over time among participants with high levels of optimism, $\beta$ $=-.27 p<.01$, but not among participants, with low levels of optimism, $\beta=.13, p=.22$. Moreover, optimism was significantly associated with higher increase in positive affect among participants with low caregiver burden, $\beta=.33, p<.01$, but not among their counterparts with high levels of caregiver burden, $\beta=-.06, p=.46$.

\section{The Mediating Role of Coping}

To examine whether the use of care-specific coping statistically explained the observed effects of dispositional optimism on participants' subjective well-being, we first identified potential mediators by considering those coping strategies that were associated with optimism (i.e., active coping, planning, behavioral disengagement, positive 
reframing, acceptance, self-blame, denial and substance use, see Table 3), and that were significantly associated with the respective outcomes. In this regard, Table 4 presents the associations between baseline levels of coping, and baseline and follow-up levels of caregiver burden, depressive symptoms, purpose in life, satisfaction with life, positive affect and negative affect. The results indicate that self-blame and denial were significantly associated with burden and with all the subjective well-being outcomes, and therefore qualified as potential mediators of the effects of optimism on these outcomes. Behavioral disengagement, positive reframing and substance use were significantly associated with depressive symptoms, purpose in life, satisfaction with life, positive and negative affect, thus can be qualified as potential mediators of the effects of optimism on these well-being indicators. Acceptance was significantly associated with depressive symptoms, purpose in life, and positive and negative affect, therefore qualified as a potential mediator of optimism on these outcomes. Active coping was significantly associated with depressive symptoms, purpose in life and positive affect, and therefore qualified as a potential mediator of the effects of optimism on these outcomes. Lastly, planning was significantly associated with purpose in life and positive affect, and therefore qualified as a potential mediator of the effects of optimism on these outcomes. Exploration of the potential mediating effect of the previously identified coping behaviors in the cross sectional analyses (T1) revealed the following: including the seven coping behaviors that could potentially mediate the association between optimism and depressive symptoms revealed that only acceptance had a significant direct effect on depressive symptoms $(\beta=-18, p=.03)$, and Sobel test confirmed a significant mediation effect $(Z=-2.07, p=.04)$. These findings suggest that optimists accepted their 
caregiving-related problems to a greater extent than pessimists, which explained their lower levels of depressive symptoms.

Including the five coping behaviors that could potentially mediate the association between optimism and negative affect, revealed a significant mediation effect of selfblame $(\beta=.28, p<.01$, Sobel test: $Z=-2.65, p<.01)$. These findings suggest that pessimists tend to blame themselves more frequently for caregiving-related problems than optimists, which explain their elevated levels of negative affect.

Including the seven coping behaviors that could potentially mediate the association between optimism and purpose in life, showed a marginal effect of denial on these associations $(\beta=-.17, p=.04$; Sobel test: $Z=1.67, p=.09)$. This suggests that optimists used denial to cope with caregiving stress less frequently than pessimists, which explain their higher levels of purpose in life.

Of the two potential coping strategies that could mediate the association between optimism and burden (self-blame and denial), only self-blame had a significant mediating effect $(\beta=.22, p<.01$; Sobel test: $Z=-2.20, p=.03)$. These finding suggests that optimists resort less frequently to self-blame, which explains their lower levels of burden. No coping behaviors were found to mediate the effect of optimism on satisfaction with life or positive affect.

In the longitudinal analyses (T2), there was no main effect of optimism on change of any of the well-being outcomes. However, there was a significant interaction effect of burden and optimism on satisfaction with life and on positive affect. Exploring the coping behavior that could potentially mediate the association between optimism and satisfaction with life revealed that positive reframing and acceptance could be potential mediators ( $\beta$ 
$=.22, p<.01, \beta=-.19, p=.04$ respectively). However, Sobel test revealed no significant mediating effect of neither positive reframing $(Z=1.00, p=.31)$ nor acceptance $(Z=-$ $0.45, p=.65)$.

The exploration of the potential mediating effect that coping behaviors may have on the association between the interaction of burden with optimism on positive affect, revealed no significant mediating effect of any of the coping behaviors.

\section{Unmitigated communion}

To examine whether unmitigated communion is associated with participants' well-being, two sets of cross-sectional and longitudinal regression analyses were performed. The results are presented in Table 10 and Table 11. In these analyses, first baseline levels and follow-up levels (controlling for baseline levels) of caregiver burden, depressive symptoms, purpose in life, satisfaction with life, positive and negative affect were predicted, by participants' baseline levels of unmitigated communion (Model 1). Subsequently, caregiver burden was examined in order to determine whether it could explain the obtained effects on participants' general subjective well-being. This was accomplished by repeating the analyses for predicting indicators of subjective well-being, and additionally including baseline levels of caregiver burden into these regression analyses (Model 2). Next, the interaction term between caregiver burden and unmitigated communion was included into these analyses to examine whether unmitigated communion can moderate the associations between caregiver burden and participants' general subjective well-being. Finally, the mediation effects that care specific coping behaviors may have on the associations between unmitigated communion and indicators of subjective well-being was explored. All analyses statistically controlled for the 
Table 10

Cross-sectional results from hierarchical regression analyses predicting baseline levels of depressive symptoms, purpose in life, satisfaction with life, positive and negative affect, and caregiver burden, by participants' unmitigated communion (Model 1) and by the interactions between caregiver burden and unmitigated communion (Model 2)

\begin{tabular}{|c|c|c|c|c|c|c|c|c|c|c|c|c|}
\hline & \multicolumn{2}{|c|}{$\begin{array}{l}\text { Depressive } \\
\text { symptoms }\end{array}$} & \multicolumn{2}{|c|}{$\begin{array}{l}\text { Purpose } \\
\text { in life }\end{array}$} & \multicolumn{2}{|c|}{$\begin{array}{c}\text { Satisfaction } \\
\text { with life }\end{array}$} & \multicolumn{2}{|c|}{$\begin{array}{l}\text { Positive } \\
\text { affect }\end{array}$} & \multicolumn{2}{|c|}{$\begin{array}{l}\text { Negative } \\
\text { affect }\end{array}$} & \multicolumn{2}{|c|}{$\begin{array}{l}\text { Caregiver } \\
\text { burden }\end{array}$} \\
\hline & $\mathrm{R}^{2}$ & $\beta$ & $\mathrm{R}^{2}$ & $\beta$ & $\mathrm{R}^{2}$ & $\beta$ & $\mathrm{R}^{2}$ & $\beta$ & $\mathrm{R}^{2}$ & $\beta$ & $\mathrm{R}^{2}$ & $\beta$ \\
\hline \multicolumn{13}{|l|}{ Model 1} \\
\hline Unmit' com' (UC) & .01 & .12 & 0 & -.04 & .02 & -.16 & 0 & -.01 & $.03 *$ & $.18^{*}$ & .01 & .09 \\
\hline \multicolumn{13}{|l|}{ Model 2} \\
\hline Burden & $.07 * *$ & $.29 * *$ & $.03 *$ & $-.19 *$ & $.13 * *$ & $-.39 * *$ & 0 & -.07 & $.08 * *$ & $.30 * *$ & & \\
\hline Unmit' com' (UC) & .01 & .09 & 0 & -.02 & .01 & -.12 & 0 & -.01 & .02 & .15 & & \\
\hline \multicolumn{13}{|l|}{ Interactions } \\
\hline Burden X UC & .01 & .10 & 0 & -.02 & .01 & -.09 & .01 & -.10 & 0 & .07 & & \\
\hline
\end{tabular}

Note: Analyses were controlled for caregiver's age, sex, education, partnership status, and chronic health problems, and time since care recipient's first diagnosis and illness severity. $\mathrm{R}^{2} \mathrm{~s}$ for predictors represent unique amount of variance explained in the outcomes.

$N=147, * p<.05 ; * * p<.01$ 
previously described covariates, and the predictor variables were centered prior to conducting the analyses.

\section{Cross-sectional analyses}

The cross-sectional analyses of the associations between unmitigated communion, caregiver burden, and indicators of subjective well-being revealed a main effect of unmitigated communion on baseline levels of negative effect (Table 10, Model 1). Participants with high levels of unmitigated communion manifested a higher level of negative affect than their counterparts with low unmitigated communion $F(1,138)=4.68$, $\beta=.18, R^{2}=.03, p=.03$. No other main effects were found. The subsequent inclusion of baseline levels of caregiver burden into the model (Table 10, Model 2) demonstrated that caregiver burden was significantly associated with higher baseline levels of depressive symptoms $F(1,137)=13.83, p<.01$, and negative affect $F(1,137)=13.22$, $p<.01$ and with lower levels of purpose in life $F(1,137)=4.72, p=.03$ and satisfaction with life $F(1,137)=23.51, p<.01$. The inclusion of burden rendered the main effect of unmitigated communion on negative affect non-significant, however Sobel test revealed no mediating effect of burden on the association between unmitigated communion and negative affect $(Z=1.35, p=0.18)$. Finally, no significant interaction effects were found between caregiver burden and unmitigated communion on indicators of subjective wellbeing.

\section{Longitudinal analyses}

The results from the longitudinal analyses are presented in Table 11 and show that baseline levels of the outcomes were significantly associated with follow-up levels of participants' caregiver burden, depressive symptoms, purpose in life, satisfaction with 


\section{Table 11}

Longitudinal results from hierarchical regression analyses predicting baseline levels of depressive symptoms, purpose in life, satisfaction with life, positive and negative affect, and caregiver burden, by participants' unmitigated communion (Model 1) and by the interactions between caregiver burden and unmitigated communion (Model 2)

\begin{tabular}{|c|c|c|c|c|c|c|c|c|c|c|c|c|}
\hline & \multicolumn{2}{|c|}{$\begin{array}{c}\text { T2 Depressive } \\
\text { symptoms }\end{array}$} & \multicolumn{2}{|c|}{$\begin{array}{c}\text { T2 Purpose in } \\
\text { life }\end{array}$} & \multicolumn{2}{|c|}{$\begin{array}{l}\text { T2 Satisfaction } \\
\text { with life }\end{array}$} & \multicolumn{2}{|c|}{$\begin{array}{l}\text { T2 Positive } \\
\text { affect }\end{array}$} & \multicolumn{2}{|c|}{$\begin{array}{c}\text { T2 Negative } \\
\text { affect }\end{array}$} & \multicolumn{2}{|c|}{$\begin{array}{c}\text { T2 Caregiver } \\
\text { burden }\end{array}$} \\
\hline & $\mathrm{R}^{2}$ & $\beta$ & $\mathrm{R}^{2}$ & $\beta$ & $\mathrm{R}^{2}$ & $\beta$ & $\mathrm{R}^{2}$ & $\beta$ & $\mathrm{R}^{2}$ & $\beta$ & $\mathrm{R}^{2}$ & $\beta$ \\
\hline \multicolumn{13}{|l|}{ Model 1} \\
\hline Tl Outcomes & $.26 * *$ & $.54 * *$ & $.32 * *$ & $.59 * *$ & $.45 * *$ & $.71 * *$ & $.50 * *$ & $.75 * *$ & $.37 * *$ & $.63 * *$ & $.53^{* *}$ & $.77 * *$ \\
\hline Unmit' com' (UC) & $.03 * *$ & $.18 * *$ & 0 & .01 & 0 & -.06 & 0 & -.07 & 0 & .04 & 0 & -.06 \\
\hline \multicolumn{13}{|l|}{ Model 2} \\
\hline T1 Outcomes & $.21 * *$ & $.51 * *$ & $.31 * *$ & $.60 * *$ & $.33 * *$ & $.67 * *$ & $.49 * *$ & $.74 * *$ & $.30 * *$ & $.59 * *$ & & \\
\hline Burden & $.02 *$ & $.15^{*}$ & 0 & .02 & .01 & -.10 & .01 & -.11 & .02 & .13 & & \\
\hline Unmit' com' (UC) & $.02 * *$ & $.17 * *$ & 0 & 0 & 0 & -.05 & 0 & -.05 & 0 & .03 & & \\
\hline \multicolumn{13}{|l|}{ Interactions } \\
\hline Burden X UC & .01 & .08 & .01 & -.10 & .01 & -.08 & 0 & -.05 & 0 & .06 & & \\
\hline
\end{tabular}

Note: Analyses were controlled for caregiver's age, sex, education, partnership status, and chronic health problems, and time since care recipient's first diagnosis and illness severity. $\mathrm{R}^{2} \mathrm{~s}$ for predictors represent unique amount of variance explained in the outcomes.

$N=121, * p<.05 ; * * p<.01$ 
life, positive and negative affect, $F_{\mathrm{s}}(1,111)>60.38$, $\beta \mathrm{s}>.54, R^{2} \mathrm{~s}>.26$, ps $<.01$.

The longitudinal analyses revealed a main effect of unmitigated communion on change in depressive symptoms over time (Model 1). Participants with high levels of unmitigated communion showed a larger increase in depressive symptoms than participants with low unmitigated communion $F(1,111)=6.66, p<.01$ The inclusion of baseline levels of burden into the analyses (Model 2) revealed a significant association between burden and increases in depressive symptoms $F(1,110)=4.83, p=.03$ The main effect of unmitigated communion on change in depressive symptoms, however, remained significant. Exploring the potential mediating effect that burden may have on the association between unmitigated communion and change in depressive symptoms revealed no such significant mediating effect $(Z=1.36, p=.17)$. No significant interaction effects were found between baseline levels of unmitigated communion and burden on the change in any of the indicators of subjective well-being overtime.

\section{The Mediating Role of Coping}

To examine whether the use of care-specific coping can statistically explain the observed effects of unmitigated communion on participants' subjective well-being, potential mediating variables were reviewed based on the previously reported correlation

analyses (see Table 3). Since there were no significant associations between unmitigated communion and any of the coping behaviors, none of these behaviors could qualify as a potential mediator of the effect unmitigated communion has on any of the well-being outcomes.

Summary of Results

The analyses presented in this section showed that personality constructs exerted 
significant effects on baseline levels and changes in subjective well-being. In addition, they demonstrated that some personality constructs exerted an indirect effect on some indicators of well-being through caregiver burden (e.g., goal disengagement on depressive symptomatology). Moreover, the results demonstrated that personality constructs can interact with caregiver burden in predicting outcomes of subjective wellbeing. Finally, certain personality constructs were found to exert a significant indirect effect on subjective well-being through certain coping behaviors (e.g., goal disengagement capacities through self-blame on negative affect). For more comprehensive summary of results, see general discussion.

\section{Personality Profiles}

It was hypothesized that personality constructs could interact with each other and that such interactions will be associated with subjective well-being. Moreover it was expected that certain personality profiles may be more adaptive than others and could predict better subjective well-being. Finally, it was predicted that such effects of personality profiles could be mediated by care-specific coping behaviors.

To examine the associations between personality profiles and participants' wellbeing, two sets of cross-sectional and longitudinal regression analyses were performed. The results are presented in Table 12 and Table 13. In these analyses, baseline levels and follow-up levels (controlling for baseline levels) of caregiver burden, depressive symptoms, purpose in life, satisfaction with life, positive, and negative affect were predicted by participants' baseline levels of goal adjustment capacities, optimism, and unmitigated communion. Subsequently two-way interaction terms between the selected personality constructs were included, to examine the effect of the different interactions on 
indicators of subjective well-being. Finally, a set of mediation analyses was conducted to examine whether effects of personality profiles on subjective well-being could be statistically explained by participants coping strategies. All analyses statistically controlled for the previously described covariates, and the predictor variables were centered prior to conducting the analyses.

\section{Cross-sectional analyses}

The results of the cross-sectional analyses are presented in Table 12 and reveal significant associations between goal disengagement capacities and positive affect, $F(1,135)=4.27, p=.04$, negative affect, $F(1,135)=6.18, p<.01$ and caregiver burden, $F(1,135)=3.84, p=.01$. More specifically, participants with high baseline levels of goal disengagement capacities experience lower levels of negative affect and caregiver burden, but also lower levels of positive affect. Goal reengagement capacities were associated with positive affect, $F(1,135)=4.76, p=.03$, and with caregiver burden, $F(1,135)=6.94, p<.01$. More specifically, participants with high goal reengagement capacities experienced higher levels of positive affect but also higher levels of caregiver burden. Dispositional optimism was significantly associated with all indicators of subjective well-being, suggesting that optimists (as compared to pessimists) experienced higher levels of purpose in life, $F(1,135)=51.50, p<.01$, satisfaction with life $F(1,135)$ $=63.15, p<.01$, and positive affect, $\mathrm{F}(1,135)=35.38, \mathrm{p}<.01$, and lower levels of depressive symptoms, $\mathrm{F}(1,135)=25.84, p<.01$, negative affect, $F(1,135)=42.17$, $p<.01$, and caregiver burden $F(1,135)=4.69, p=.03$. There were no significant associations between unmitigated communion and any indicators of subjective wellbeing. The cross-sectional analysis of the interactions between the three personality 
Table 12

Cross-sectional results from hierarchical regression analyses predicting baseline levels of indicators of subjective well-being by participants' personality constructs and their interactions

\begin{tabular}{|c|c|c|c|c|c|c|c|c|c|c|c|c|}
\hline & \multicolumn{2}{|c|}{$\begin{array}{l}\text { Depressive } \\
\text { symptoms }\end{array}$} & \multicolumn{2}{|c|}{$\begin{array}{l}\text { Purpose } \\
\text { in life }\end{array}$} & \multicolumn{2}{|c|}{$\begin{array}{l}\text { Satisfaction } \\
\text { with life }\end{array}$} & \multicolumn{2}{|c|}{$\begin{array}{l}\text { Positive } \\
\text { affect }\end{array}$} & \multicolumn{2}{|c|}{$\begin{array}{l}\text { Negative } \\
\text { affect }\end{array}$} & \multicolumn{2}{|c|}{$\begin{array}{l}\text { Caregiver } \\
\text { burden }\end{array}$} \\
\hline & $\mathrm{R}^{2}$ & $\beta$ & $\mathrm{R}^{2}$ & $\beta$ & $\mathrm{R}^{2}$ & $\beta$ & $\mathrm{R}^{2}$ & $\beta$ & $\mathrm{R}^{2}$ & $\beta$ & $\mathrm{R}^{2}$ & $\beta$ \\
\hline \multicolumn{13}{|l|}{ Main effect } \\
\hline Goal dis' (GD) & .01 & -.12 & 0 & -.04 & .01 & .12 & $.02 *$ & $-.17 *$ & $.03 * *$ & $-.19 * *$ & $.02 *$ & $-.17 *$ \\
\hline Goal re' (GR) & 0 & .05 & .02 & .15 & 0 & -.05 & $.03 *$ & $.18 *$ & .00 & .06 & $.04 * *$ & $.23 * *$ \\
\hline Optim’ (OPT) & $.16 * *$ & $-.43 * *$ & $.24 * *$ & $.52 * *$ & $.26 * *$ & $.55 * *$ & $.14 * *$ & $.39 * *$ & $.19 * *$ & $-.47 * *$ & $.03 *$ & $-.18 *$ \\
\hline Un' com' (UC) & .01 & .07 & 0 & -.01 & .01 & -.10 & 0 & -.02 & .01 & .12 & 0 & .05 \\
\hline \multicolumn{13}{|l|}{ Interactions } \\
\hline GD X GR & .01 & -.10 & 0 & -.03 & .01 & -.13 & .02 & .15 & $.02 *$ & $.16 *$ & & \\
\hline GD X OPT & $.02 *$ & $-.14 *$ & 0 & -.02 & .01 & .11 & .02 & .14 & 0 & .05 & & \\
\hline GD X UC & .01 & -.11 & 0 & .04 & .01 & .09 & 0 & 0 & 0 & .02 & & \\
\hline GR X OPT & 0 & -.02 & 0 & -.01 & 0 & -.04 & 0 & .01 & 0 & .07 & & \\
\hline GR X UC & 0 & .02 & 0 & .06 & .01 & .12 & 0 & -.01 & .01 & .07 & & \\
\hline OPT X UC & .01 & -.09 & 0 & .01 & 0 & -.07 & 0 & -.04 & .01 & -.12 & & \\
\hline
\end{tabular}

Note: Analyses were controlled for caregiver's age, sex, education, partnership status, and chronic health problems, and time since care recipient's first diagnosis and illness severity. $\mathrm{R}^{2} \mathrm{~s}$ for predictors represent unique amount of variance explained in the outcomes.

$N=147 . * p<.05 ; * * p<.01$ 
constructs and their associations with indicators of subjective well-being (see Table 12) revealed two significant interaction effects: a significant interaction effect of goal disengagement and optimism on depressive symptomatology $F(1,133)=4.48, p=.04$, and a significant interaction effect of goal disengagement and goal reengagement on negative affect $F(1,133)=5.05, p=.03$

The interaction effect of goal disengagement capacity and optimism on levels of depressive symptoms is illustrated in Figure 7, by plotting the associations between caregiver' disengagement capacities and depressive symptoms, separately for participants with low versus high levels of optimism (one standard deviation above and below the sample means). The obtained pattern of results indicated that elevated baseline levels of depressive symptoms were obtained particularly among pessimists, independent of their disengagement capacities. By contrast, optimists experienced reduced levels of baseline depressive symptoms particularly if they were able to disengage from unattainable goals, but not if they had difficulty disengaging from such goals. Analyses of simples slopes demonstrated that disengagement capacity was significantly associated with reduced levels of depressive symptoms among optimists, $\beta=-.32 p<.01$, but not among pessimists, $\beta=-.05, p=.69$. Moreover, optimism was significantly associated with lower levels of depressive symptoms in both participants with high disengagement capacity $\beta=-.58, p<.01$, and low disengagement capacity, $\beta=-.31, p<.01$.

The interaction effect of goal disengagement and goal reengagement on negative affect is illustrated in Figure 8, by plotting the associations between caregiver' disengagement capacity and negative affect, separately for participants with low versus high reengagement capacity (one standard deviation above and below the sample means). 


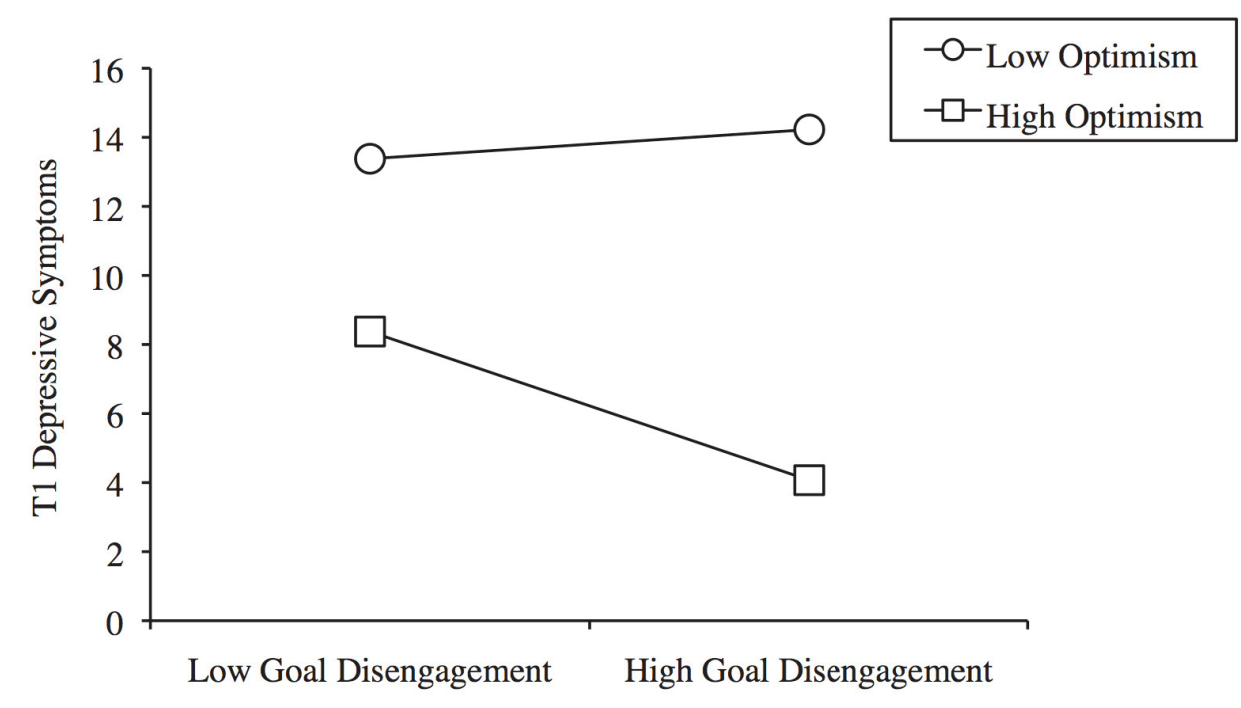

Figure 7. Associations between baseline levels of goal disengagement and depressive symptoms, separately for participants with high versus low optimism 


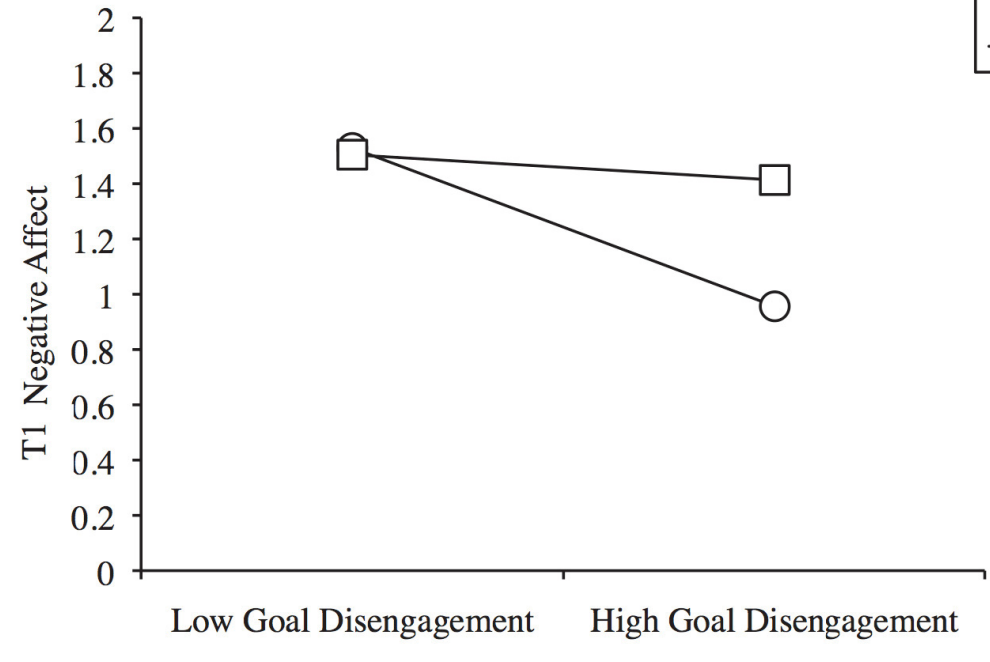

- - Low Goal Reengagement

$\neg-$ High Goal Reengagement

Figure 8. Associations between baseline levels of goal disengagement and negative affect, separately for participants with high versus low goal reengagement 
The obtained pattern of results indicated that low baseline levels of negative affect were obtained particularly among participants with high goal disengagement capacity and low reengagement capacity at baseline. By contrast, participants with high goal disengagement and high goal reengagement capacities experienced higher baseline levels of negative affect comparable to participants with low goal disengagement capacities. Analyses of simples slopes demonstrated that goal disengagement capacities were significantly associated with reduced levels of negative affect among participants who had low levels of goal reengagement capacities, $\beta=-.43, p<.01$, but not among participants with high levels of goal reengagement capacities, $\beta=-.17, p=.14$. Goal reengagement capacities were not significantly associated with reduced negative affect neither in participants with high disengagement capacities $\beta=.17, p=.26$, nor in participants with low disengagement capacities, $\beta=-.09, p=.35$.

\section{Longitudinal analyses}

The results from the longitudinal analyses are presented in Table 13 and show that baseline levels of the outcomes were significantly associated with follow-up levels of participants' caregiver burden, depressive symptoms, purpose in life, satisfaction with life, positive and negative affect, $F \mathrm{~s}(1,112)>62.29, \beta \mathrm{s}>.57, R^{2} \mathrm{~s}>.30, p s<.01$.

The longitudinal analyses reveal two significant main effects. A significant association between goal reengagement capacity and change in satisfaction with life over time, $F(1,108)=3.90, p=.05$, and a significant association between unmitigated communion and change in depressive symptoms over time, $F(1,108)=6.27, p<.01$.

These findings suggest that participants with high (as compared to low) goal 


\section{Table 13}

Longitudinal results from hierarchical regression analyses predicting baseline levels of indicators of subjective well-being by participants' personality constructs and their interactions

\begin{tabular}{|c|c|c|c|c|c|c|c|c|c|c|c|c|}
\hline & \multicolumn{2}{|c|}{$\begin{array}{l}\text { T2 Depressive } \\
\text { symptoms }\end{array}$} & \multicolumn{2}{|c|}{$\begin{array}{l}\text { T2 Purpose in } \\
\text { life }\end{array}$} & \multicolumn{2}{|c|}{$\begin{array}{l}\text { T2 Satisfaction } \\
\text { with life }\end{array}$} & \multicolumn{2}{|c|}{$\begin{array}{c}\text { T2 Positive } \\
\text { affect }\end{array}$} & \multicolumn{2}{|c|}{$\begin{array}{c}\text { T2 Negative } \\
\text { affect }\end{array}$} & \multicolumn{2}{|c|}{$\begin{array}{c}\text { T2 Caregiver } \\
\text { burden }\end{array}$} \\
\hline & $\mathrm{R}^{2}$ & $\beta$ & $\mathrm{R}^{2}$ & $\beta$ & $\mathrm{R}^{2}$ & $\beta$ & $\mathrm{R}^{2}$ & $\beta$ & $\mathrm{R}^{2}$ & $\beta$ & $\mathrm{R}^{2}$ & $\beta$ \\
\hline T1 Outcomes & $.30 * *$ & $.57 * *$ & $.32 * *$ & $.59 * *$ & $.46 * *$ & $.72 * *$ & $.50 * *$ & $.75 * *$ & $.39 * *$ & $.63 * *$ & $.53 * *$ & $.76 * *$ \\
\hline \multicolumn{13}{|l|}{ Main effect } \\
\hline Goal dis' (GD) & 0 & -.05 & .01 & -.13 & 0 & -.03 & 0 & .01 & 0 & -.03 & 0 & .02 \\
\hline Goal re' (GR) & .01 & .13 & .01 & .10 & $.01 *$ & $-.14 *$ & 0 & .01 & 0 & .05 & 0 & -.05 \\
\hline Optim' (OPT) & .01 & -.09 & .01 & .10 & 0 & .07 & .01 & .09 & 0 & .03 & 0 & 0 \\
\hline Un’ com' (UC) & $.03 * *$ & $.18 * *$ & 0 & -.02 & 0 & -.06 & 0 & -.06 & 0 & .04 & 0 & -.05 \\
\hline \multicolumn{13}{|l|}{ Interactions } \\
\hline GD X GR & 0 & .06 & 0 & .08 & 0 & 0.1 & .01 & .09 & 0 & .07 & & \\
\hline GD X OPT & 0 & .02 & $.02 *$ & $.16 *$ & 0 & .06 & 0 & .06 & .02 & -.14 & & \\
\hline GD X UC & 0 & -.02 & $.03 *$ & $-.19 *$ & 0 & -.01 & 0 & -.01 & 0 & .03 & & \\
\hline GR X OPT & 0 & .03 & 0 & -.05 & .01 & -.09 & 0 & .01 & 0 & .04 & & \\
\hline GR X UC & 0 & .06 & .02 & -.14 & 0 & -.06 & 0 & -.04 & $.02 *$ & $.16^{*}$ & & \\
\hline OPT X UC & 0 & -.05 & .02 & -.13 & .01 & .09 & .01 & -.10 & 0 & .05 & & \\
\hline
\end{tabular}

Note: Analyses were controlled for caregiver's age, sex, education, partnership status, and chronic health problems, and time since care recipient's first diagnosis and illness severity. $\mathrm{R}^{2} \mathrm{~s}$ for predictors represent unique amount of variance explained in the outcomes. $N=121, * p<.05 ; * * p<.01$ 
reengagement capacities experienced declines in satisfaction with life over time and those with high (as compared to low) unmitigated communion experienced increases of depressive symptomatology over time.

The longitudinal analyses of the interactions between the three personality constructs and their associations with indicators of subjective well-being revealed three significant interaction effects: goal disengagement significantly interacted with optimism, $F(1,106)=4.39 p=.04$, and with unmitigated communion $F(1,106)$ $=5.89, p=.02$ in predicting changes in purpose in life over time. In addition, goal reengagement and unmitigated communion exerted a significant interaction effect on changes in negative affect over time, $F(1,106)=4.63, p=.03$ (see Table 13).

The interaction effect between goal disengagement capacities and optimism on changes in purpose in life is illustrated in Figure 9, employing the previously described procedures. The observed pattern of interaction suggests that declines in purpose in life over time were obtained particularly among participants with high disengagement capacities and low-optimism at baseline. By contrast, optimist participants with high goal disengagement capacities experienced less declines in purpose in life over time, comparable with those low on disengagement capacities. Analyses of simples slopes demonstrated that goal disengagement capacities were significantly associated with reduced levels of purpose in life among pessimists, $\beta=$ $-.29, p=.02$, but not among optimists, $\beta=.02, p=.86$. Moreover, optimism was significantly associated with lower levels of purpose in life but only in participants with high disengagement capacities, $\beta=.29, p=.02$, and not in participants with 


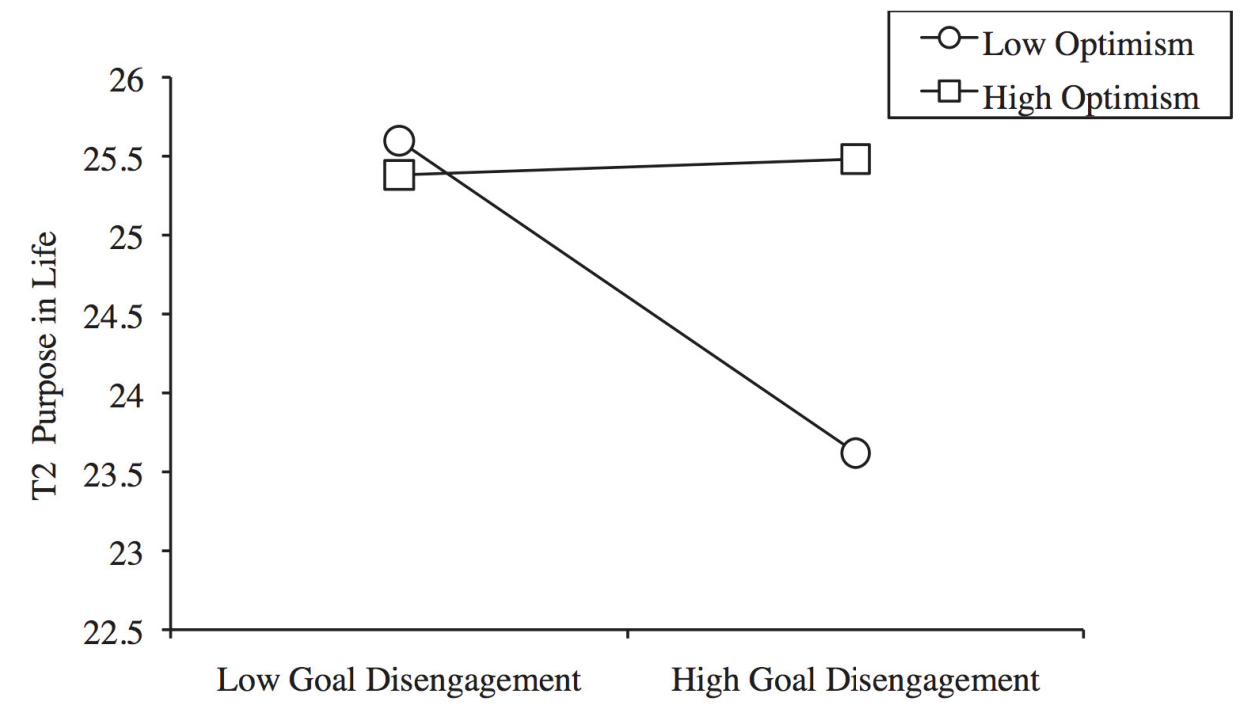

Figure 9. Associations between baseline levels of goal disengagement and change in purpose in life, separately for participants with high versus low optimism 
low disengagement capacities, $\beta=-.03, p=.82$.

The interaction effect between goal disengagement capacities and unmitigated communion on changes in purpose in life is illustrated in Figure 10, employing the previously described procedures. The observed pattern of interaction suggests that declines in purpose in life over time were obtained particularly among participants with high disengagement capacities and high unmitigated communion. By contrast, participants with high disengagement capacities and low unmitigated communion experienced less declines in purpose in life comparable to participants with low disengagement capacities. Analyses of simples slopes demonstrated that disengagement capacities were significantly associated with reduced level of purpose in life among participants who had high levels of unmitigated communion, $\beta=-.33 p<.01$, but not among participants with low levels of unmitigated communion, $\beta=.03, p=.78$. Moreover, unmitigated communion was significantly associated with lower levels of purpose in life in participants with high disengagement capacity, $\beta=-.24, p=.05$, but not in participants with low disengagement capacity, $\beta=.12, p=.21$.

The interaction effect between goal reengagement capacities and unmitigated communion on changes in negative affect is illustrated in Figure 11, employing the previously described procedures. The observed pattern of interaction suggests that increases in negative affect were obtained among participants with high goal reengagement capacities and high unmitigated communion. By contrast, 


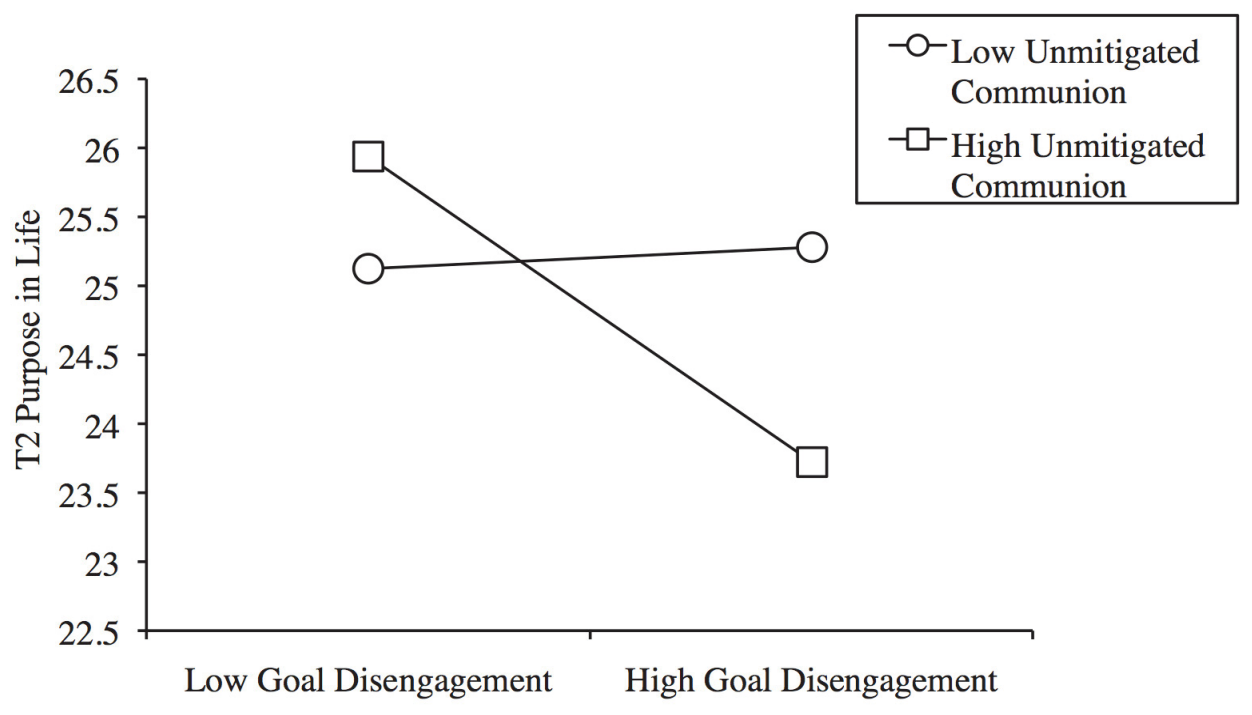

Figure 10. Associations between baseline levels of goal disengagement and change in purpose in life, separately for participants with high versus low unmitigated communion 


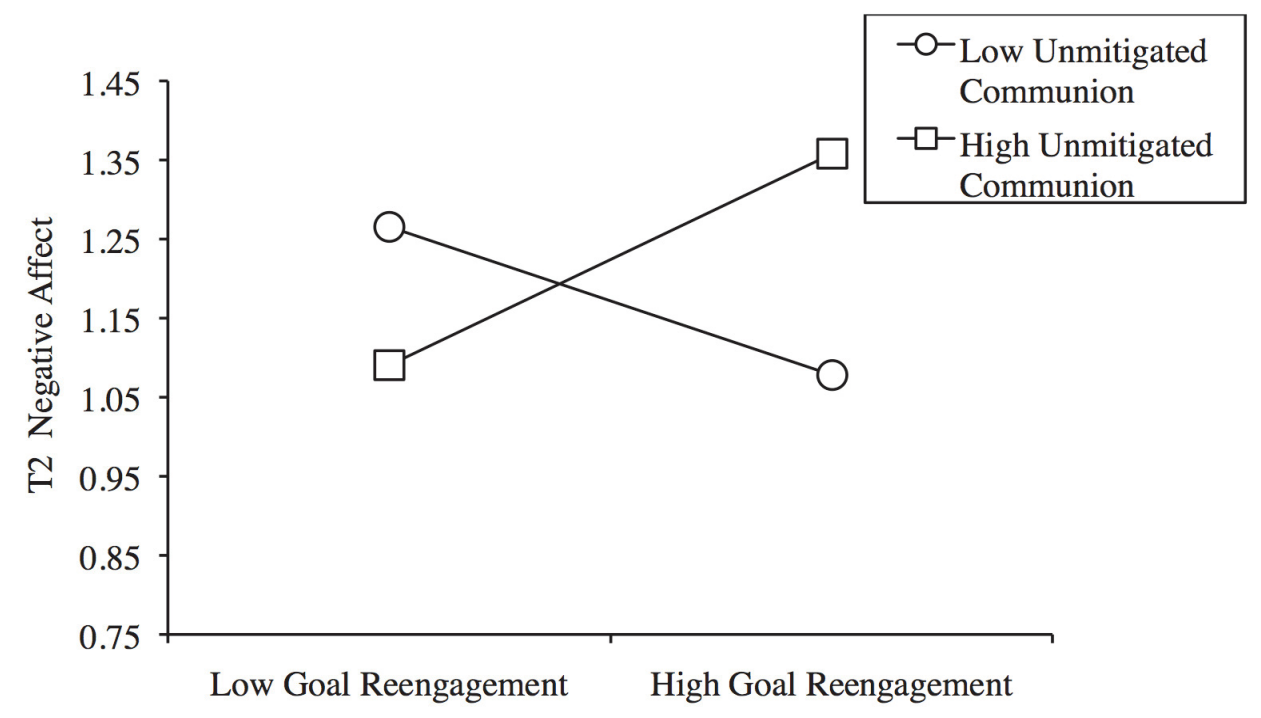

Figure 11. Associations between baseline levels of goal reengagement and change in negative affect, separately for participants with high versus low unmitigated communion 
participants with high reengagement capacities and low unmitigated communion experienced smaller increases in negative affect comparable to their counterparts with low goal reengagement capacities. Analyses of simples slopes demonstrated that reengagement capacities had a marginally significant association with increases in negative affect among participants who had high levels of unmitigated communion, $\beta=.20, p=.08$, but not among participants with low levels of unmitigated communion, $\beta=-.08, p=.46$. Additionally, unmitigated communion had a marginally significant association with changes in negative affect in participants with high reengagement, $\beta=.18, p=.09$, but not low reengagement capacities, $\beta=-.09, p=.37$.

\section{The Mediating Role of Coping}

To examine whether the use of care-specific coping behaviors can statistically explain the observed effects of the personality profiles that were found to have significant associations with indicators of well-being, first, potential coping mediators were identified for these profiles; next, potential coping mediators were identified for the associations between these profiles and the outcome indicators they were significantly associated with. The four personality profiles that were previously found to have at least one significant association with at least one wellbeing outcome are goal disengagement and optimism (GDxOPT on baseline levels of depressive symptoms and on follow-up levels of purpose in life); goal disengagement and goal reengagement (GDxGR on baseline levels of negative affect); goal disengagement and unmitigated communion (GDxUC on follow-up 
levels of purpose in life); and goal reengagement and unmitigated communion (GRxUC on follow-up levels of negative affect).

To identify potential mediators of these interaction effects, the regressions for predicting indicators of subjective well-being were repeated; this time predicting each of the coping strategies. Coping strategies were selected as potential mediators if the respective interactions of the personality factors significantly predicted the coping strategies. These analyses revealed the following potential mediators: 1 . The interaction between GDxOPT predicted substance use $\mathrm{F}(1,133)=6.48, \beta=.21 p=$ $.01, \mathrm{R}^{2}=.04$, and therefore could mediate the association between GDxOPT and depressive symptoms. 2. The interaction between GDxGR predicted positive reframing $\mathrm{F}(1,133)=3.99, \beta=-.16 p=.05, \mathrm{R}^{2}=.02$, and self-blame $\mathrm{F}(1,133)=$ $5.56, \beta=.19 p=.02, \mathrm{R}^{2}=.03$, and therefore could mediate the association between GDxGR and negative affect. 3. The interaction between GDxUC predicted active coping $\mathrm{F}(1,107)=4.35, \beta=-.19 p=.04, \mathrm{R}^{2}=.03$; positive reframing $\mathrm{F}(1,107)=$ $5.06, \beta=-.20 p=.03, \mathrm{R}^{2}=.04 ;$ and emotional support $\mathrm{F}(1,107)=4.52, \beta=-.20 p$ $=.04, \mathrm{R}^{2}=.03$, and therefore could mediate the association between GDxUC and change in purpose in life over time. 4. Finally, the interaction between GRxUC predicted positive reframing $\left.\mathrm{F}(1,107)=4.80, \beta=-.19 p=.03, \mathrm{R}^{2}=.03\right)$ and selfblame $\mathrm{F}(1,107)=5.32, \beta=.20 p=.02, \mathrm{R}^{2}=.04$, and thus could mediate the association GRxUC and change in negative affect over time.

Subsequently conducted Sobel tests revealed only one marginally significant mediation effect: self-blame was found to have a marginal mediating effect on the association between goal disengagement and goal reengagement (GDxGR) on 
negative affect $(Z=1.86, p=.06)$. [Sobel tests did not confirm any of the other potential coping mediators: 1 . Substance use (GDxOPT on depressive symptoms) Z $=1.08, p=.28)$. 2. Positive reframing (GDxGR on negative affect), $\mathrm{Z}=1.04, p=$ 30. 3. Active coping, positive reframing and emotional support (GDxUC on change in purpose), $\mathrm{Z}=-1.26, p=.21 ; \mathrm{Z}=-.68, p=.50 ; \mathrm{Z}=-.73, p=.47$ respectively. 4 . Positive reframing and self-blame (GRxUC on change in negative affect), $\mathrm{Z}=1.53$, $p=.13 ; \mathrm{Z}=1.57, p=.12$ respectively.]

The interaction effect of goal disengagement and goal reengagement capacities on self-blame is illustrated in Figure 12, by plotting the associations between caregiver' disengagement capacities and self-blame, separately for participants with low versus high goal reengagement capacities (one standard deviation above and below the sample means). The observed pattern of interaction suggests that low baseline levels of self-blame were obtained particularly among participants with high disengagement and low reengagement capacities at baseline. By contrast, participants with high disengagement and high reengagement capacities experienced higher levels of self-blame comparable to low disengagement capacities. Analyses of simples slopes demonstrated that goal disengagement capacities were significantly associated with reduced levels of selfblame among participants with low levels of reengagement capacities $(\beta=-.36 p<$ .01) but not among participants with high levels of goal reengagement capacities ( $\beta$ $=-.03 p=.79)$. Additionally, goal reengagement capacities were not significantly associated with reduced self-blame neither among participants with high disengagement capacity ( $\beta=.23 p=.13$ ), nor among participants with low 
disengagement capacities $(\beta=-.10 p=.32)$. Combined with the findings described in figure 8 , these findings reinforce the observation that goal disengagement capacities are instrumental in reducing caregivers' negative affect and self-blame particularly among participants with poor goal reengagement capacities. It also suggests that among caregivers with poor goal reengagement capacities, those who are able to disengage experience reduced levels of negative affect at least in part because they do not tend to blame themselves for caregiving problems. 


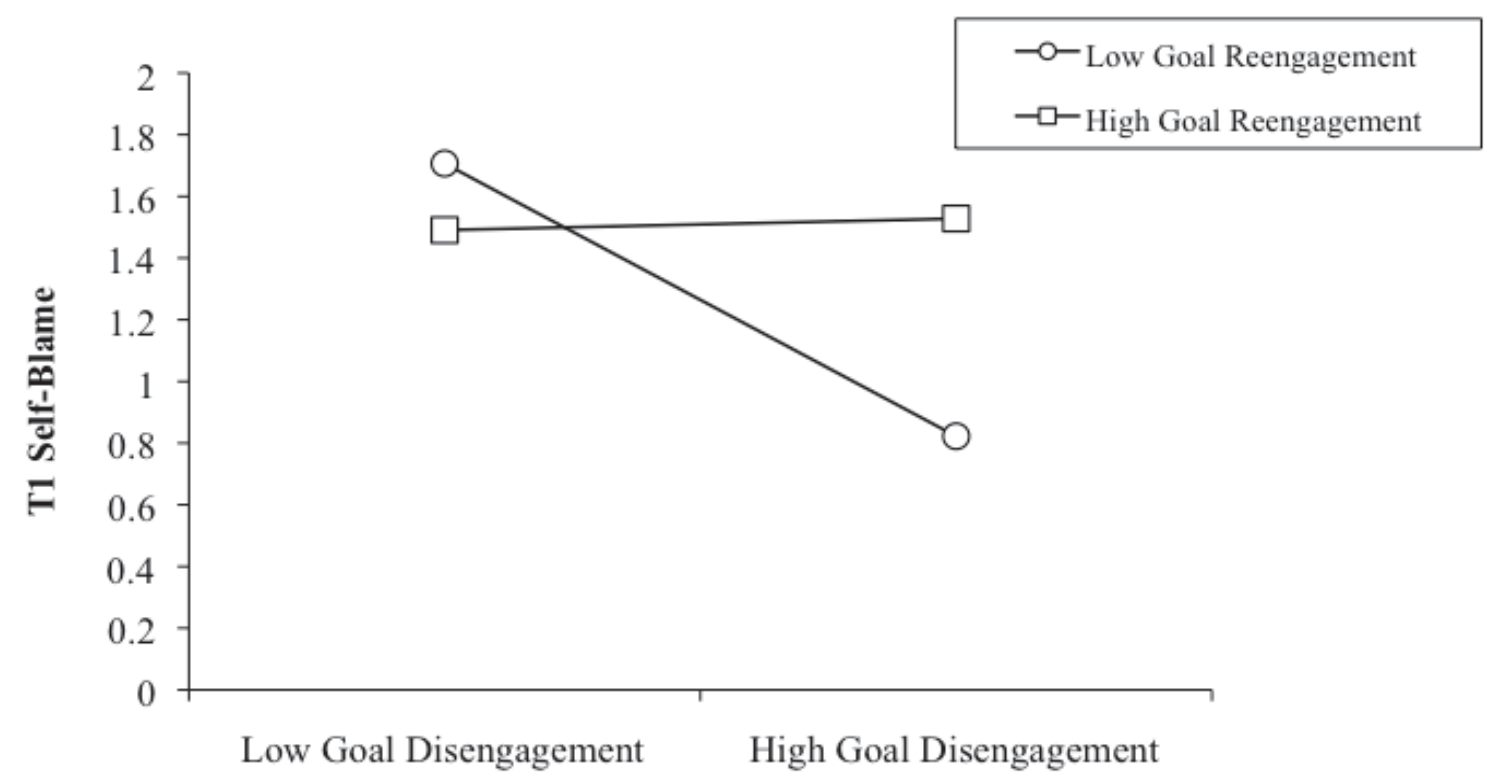

Figure 12. Associations between baseline levels of goal disengagement and selfblame separately for participants with high versus low goal reengagement capacities 


\section{Summary of Results}

The analyses presented in this section showed that some personality profiles exerted significant effects on baseline levels and changes in some of the subjective well-being outcomes (e.g., a significant interaction effect of goal disengagement capacities and optimism on depressive symptomatology). Only one personality profile was found to exert a marginally significant indirect effect on subjective wellbeing through coping behaviors (i.e., goal disengagement and goal reengagement capacities through self-blame on negative affect). For a more comprehensive description of results, see general Discussion. 


\section{Chapter 4}

\section{DISCUSSION}

This study was conducted to understand how long-term caregiving to a relative with mental illness affects the subjective well-being of the caregiver. The functional associations between personality constructs, coping behaviors and subjective well-being were examined in the context of caregiving burden. Assuming that caregiving to a family member with mental illness represents a chronic stressor, it was postulated that better subjective well-being will be present among caregivers with adaptive personality constructs. It was also expected that adaptive personality constructs would facilitate effective care-specific coping behaviors. Additionally, it was expected that personality constructs could form personality profiles, some of which may be more adaptive than others, and affect subjective well-being accordingly.

The study's findings provide substantial support to the hypotheses and indicate that in the presence of caregiving burden, adaptive personality factors are associated with higher levels of subjective well-being. For example, personality factors such as goal adjustment capacities and optimism were associated with higher levels of subjective well-being, such as satisfaction with life and positive affect.

There was also evidence that the influence of personality constructs on subjective well-being was exerted, in certain cases, through care-specific coping behaviors that were facilitated by these personality constructs. These coping behaviors were found to mediate the associations between personality constructs 
and indicators of subjective well-being, and served to moderate (or buffer) the effect of caregiving burden on well-being outcomes.

Additionally, there was evidence that personality profiles that were created by combinations of personality constructs were associated with subjective wellbeing. However, some personality constructs were found to be more effective than others, and some even exerted a mal-adaptive influence on well-being.

In the following sections, the specific results are discussed separately for each personality construct. This is followed by an evaluation of the influence of personality profiles, the implications of the findings for theory and practice, and the limitations of the study and its implications for future research.

\section{Goal Adjustment Capacities}

The present study showed that goal adjustment capacities are associated with how individuals cope with the stressful situation of caregiving for a family member with mental illness. In addition, goal adjustment capacities predicted levels of, and changes in, indicators of subjective well-being. Finally, the effects of goal adjustment capacities on subjective well-being were statistically explained by the adoption of specific coping strategies. This pattern of findings suggests that coping represents a unique mechanism that links goal adjustment capacities and subjective well-being in the context of a chronic stressor.

More specifically, the study's results showed that compared with participants who exhibited poor goal disengagement capacities, participants who were better able to disengage from unattainable goals blamed themselves less frequently for problems associated with caregiving. In addition, caregivers with 
better goal disengagement capacities experienced lower levels of negative affect, as well as lower levels of caregiver burden that mediated their lower levels of depressive symptoms. Additionally, compared with their counterparts with poor disengagement capacities, highly burdened caregivers with good goal disengagement capacities experienced higher levels of satisfaction with life.

The longitudinal analyses further demonstrated that caregivers who were highly burdened at baseline experienced particularly significant increases in both depressive symptom and negative affect over time, but only if they had difficulty disengaging from unattainable goals, and not if they were able to abandon unattainable goals. Finally, the cross-sectional effects of adaptive goal disengagement were statistically explained by a less frequent use of self-blame, and the longitudinal buffering effect of adaptive goal disengagement capacities was partially mediated by a less frequent use of substances linked to changes of depressive symptoms over time and self-blame linked to change in negative affect over time.

These findings are consistent with the hypothesis that individual differences in goal adjustment capacities shape the ways that people manage and respond to stressful caregiving situations. In particular, individuals who have difficulty abandoning unattainable goals may deplete their self-regulatory resources, and as a result feel overwhelmed and perceive that they could and should have devoted more time and energy to caring for their family member. As a consequence, they are more likely to blame themselves for problems that arise and to use substances to cope with the associated emotional difficulties (Newcomb \& Harlow, 1986). In other 
work, these coping strategies (i.e., self-blame and substance use) have been linked with negative emotional states, including depressive symptoms (Abramson, Seligman, \& Teasdale, 1978; Hasin \& Grant, 2002). Thus, goal disengagement capacities play a role in shaping how caregivers manage stressful situations and their ensuing levels of subjective well-being.

The effects of goal reengagement on caregivers' coping and well-being were mixed and suggest intriguing speculations. For example, the capacity to reengage in other new goals was associated with both more and less effective coping strategies (e.g., planning, positive reframing, and religion vs. venting and self-distraction, see Table 3). Moreover, in the cross-sectional analyses goal reengagement capacities were related to higher purpose in life and higher positive affect, but also to greater caregiver burden. Mediation analyses clarified the basis for these seemingly contradictory patterns, highlighting the role that specific coping strategies played in fostering different outcomes. Specifically, the analyses suggested that goal reengagement had beneficial effects on purpose in life and positive affect through the promotion of more frequent use of positive reframing. By contrast, the more undesirable effects of goal reengagement on increased levels of caregiver burden were mediated by a more frequent use of venting and self-distraction. These findings suggest that pursuing new goals represents a double-edged sword in chronically stressful situations like caregiving. On the one hand, pursuing new goals can facilitate the reappraisal of problematic situations in a more positive light and thus foster a general purpose in life (Folkman \& Moskowitz, 2000) and positive affect. On the other hand, however, it may also distract a person from addressing the 
primary stressor and elevate the person's caregiving burden.

It is noted that goal disengagement capacities were not associated with purpose in life and with positive affect. This finding is consistent with previous research documenting that goal disengagement capacities are often more strongly associated with negative, as compared to positive, indicators of subjective wellbeing (Wrosch et al., 2007). In addition, it seems important to address the different patterns of cross-sectional and longitudinal findings. In particular, the analyses did not confirm associations between goal reengagement capacities and indicators of subjective well-being over time. Such associations may not have been observed in the present study because the selection, pursuit, and attainment of new goals can take a considerable period of time (Heckhausen, 1991), and the beneficial or detrimental effects of new goal pursuits may thus only be determined in lengthy follow-ups. Consistent with this possibility, research among older adults showed that goal reengagement capacities buffered an adverse effect of stressors on increases in depressive symptoms only after 4 years, but not after 2 years (Dunne \& Wrosch, 2009).

In sum, goal disengagement capacities facilitated care-specific coping behaviors that led to better subjective well-being. This was possible by both reducing negative outcomes and by enhancing positive outcomes. On the other hand goal reengagement capacities were associated with better subjective well-being (through certain care-specific coping behaviors), but also with heighten burden. This suggests that while abandoning unattainable goals can be beneficial in dealing with caregiver burden, the search for new goals as a response to caregiving stress 
may act as a double-edge sword: it can enhance some indicators of subjective wellbeing but at the same time it can compromise others.

\section{Dispositional Optimism}

The present study showed that optimism can also be associated with how individuals cope with the stressful situation of caregiving for a family member with mental illness. In addition, optimism predicted levels of, and changes in, indicators of subjective well-being. Finally, the effects of optimism on subjective well-being were statistically explained by the adoption of care-specific coping strategies. This pattern of findings suggests that coping also links optimism and subjective wellbeing in the context of a chronic stressor.

More specifically, the cross-sectional results showed that optimistic participants experienced lower levels of depressive symptoms, negative affect and burden, as well as higher levels of purpose in life, satisfaction with life, and positive affect. Although the main effects of optimism remained when burden was included in the analyses, the reported results suggest that burden had a marginal indirect effect on the associations between optimism and indicators of subjective well-being.

Optimism was found to have positive associations with effective carespecific coping behaviors (i.e., active coping, planning, positive reframing, acceptance) and negative associations with less effective coping behaviors (i.e., behavioral disengagement, self-blame, denial and substance use). The importance of care-specific coping behaviors as mediators in the cross sectional analyses was reflected in the following findings: 1 . Responding to stressful caregiving situations with acceptance, explained the association between optimism and lower caregivers' 
depression. 2. Infrequent self-blame explained the association between optimism and lower negative affect, as well as between optimism and lower burden. 3 . Infrequent use of denial explained the association between optimism and higher levels of purpose in life. No care-specific coping behaviors were found to mediate the associations between optimism and satisfaction with life, and between optimism and positive affect.

These findings suggest that optimists who tend to accept stressful situations without denying, ignoring or blaming themselves, experience higher levels of subjective well-being, such as enhanced purpose in life, or lower levels of depressive symptoms and burden. The associations between optimism and satisfaction with life, and between optimism and positive affect may be explained by different pathways.

The longitudinal analyses demonstrated that increases in satisfaction with life, as well as increases in positive affect over time, were obtained particularly among optimistic caregivers who experienced low levels of burden. However, when burden levels were high, optimistic caregivers did not experience better well-being than their pessimistic counterparts (see Figure 5 and 6). This suggests that when burden is low, optimism matters and helps caregivers take better care of themselves. However, when burden is high, the tendency of optimistic caregivers to persist on goal attainment may stretch them too thin, thus may become counter-productive. When burden is high, therefore, optimism alone may not be sufficient in protecting the caregiver's well-being. As previously discussed, in the face of high burden, abandoning some goals may be more useful because it frees resources that can be 
used to manage the burdensome caregiving situation.

No care-specific coping behaviors were found to mediate the interaction effect of optimism and burden in predicting satisfaction with life or positive affect in the longitudinal analyses. While positive reframing and acceptance qualified as potential mediators, none of these variables was found to have an indirect effect on these associations. Thus, while it could be speculated that positive reframing and acceptance may characterize, at least in part, the behaviors of optimistic caregivers, as mentioned earlier, satisfaction with life and positive affect may represent a different type of outcomes which may not be associated with care-specific coping behaviors in the same way as other outcomes, such as purpose in life. It is possible, for example, that optimism exerts an influence on some well-being outcomes through different pathways, such as social networks. Earlier findings pointed to the association between optimism and better social networks in predicting well-being (Brissette et al., 2002; Carver et al., 2010). This variable was not explored in this study and may explain the absence of mediation effect of coping in relation to these outcomes.

These findings are consistent with the notion that optimists face adversity differently than pessimists (Carver et al., 2010) and that optimism is linked with resiliency to stress and thus serves as a protective personality factor for mental and physical health (Lorant et al., 2003). Other studies have shown that optimists typically face stressful situations heads-on, without denial, and without blaming themselves for these situations. Optimists also try harder than pessimists to attain their goals and overcome problems (Scheier \& Carver, 1985). Less denial was 
found in optimists who were recovering from a coronary bypass surgery (Scheier et al., 1989). The present study is also consistent with earlier findings that showed a link between optimism and life satisfaction (Chang, 1998), lower depression (Carver \& Gaines, 1987; Christensen et al., 1998; Given et al., 1993) and lower negative emotions (King et al., 1998). While limited, the findings offer support to the link between optimism and more active and complex strategies in dealing with stressful situations and less engagement in emotional expression (Scheier \& Carver, 1985). This study's findings are also congruent with earlier studies that showed higher levels of depression in pessimists (Christensen et al., 1998; Given et al., 1993).

In sum, the findings are consistent with earlier research that points to the beneficial role of optimism. Moreover, this study demonstrates that optimism is also adaptive in the context of caregiving to relatives with mental illness, in particular when caregiving burden is not extremely high. As well, optimism, which was found to be associated with subjective well-being outcomes in the present study, appears to be a protective factor that can mitigate, at least in part, the effect of the chronic stress which is associated with caregiving responsibilities.

\section{Unmitigated Communion}

Unlike the findings related to goal adjustment capacities and optimism, there were fewer effects of unmitigated communion on indicators of subjective wellbeing. Unmitigated communion was found to be associated only with negative affect in the cross sectional analysis, and with a change in depressive symptoms in the longitudinal analysis. Moreover, no associations were found between 
unmitigated communion and care-specific coping behaviors, therefore the mechanism by which unmitigated communion exerts an influence on subjective well-being could not be explained by coping in this study.

More specifically, participants who care about other individuals' needs to the extent that they compromise their own needs expressed more negative affect and experienced larger increases in depressive symptoms over time than caregivers with low levels of unmitigated communion. However, while levels of caregiving burden rendered the main effect of unmitigated communion on negative affect nonsignificant in the cross sectional analysis, it was not found to have a significant indirect effect on this association. As well, the main effect of unmitigated communion on increases in depressive symptoms over time remained when burden was included in the analysis. This suggests that an increase in depression may not be related just to caregiving burden, but may be rooted in the personality construct itself and other associated specific behaviors. In this regard, it is also possible that, for example, unlike optimism, unmitigated communion can be perceived as having value, suggesting that what may be considered over-involvement to an outsider, may be perceived as good parenting by the high unmitigated communion caregivers. Thus, it can be speculated that caregivers with high levels of unmitigated communion are so heavily immersed in their caregiving role that they may have abandoned other goals they might have had for themselves, which are attainable and could provide purpose for living. Further, the remaining goals for these caregivers may be related to their caregiving role or to their ill relative, rather than to their lives outside this role, suggesting that they may have only few 
purposeful activities beyond being caregivers. Maintaining a certain level of diversity, however, has been shown to protect the well-being of individuals who encounter stressful life situations (Linville, 1987). For a more detailed discussion on the different types of goals see Limitations and future research section.

No further associations were found between unmitigated communion and other indicators of subjective well-being, including purpose in life, satisfaction with life, positive affect, and burden. Nevertheless, the analyses showed that unmitigated communion was associated with higher levels of chronic health problems, and while this effect suggests only a trend $(\mathrm{r}=.13 \cdot p=.11)$, it is congruent with earlier studies that linked unmitigated communion with compromised psychological and physical well-being (Helgeson \& Fritz, 1998). Additionally, high unmitigated communion participants were found to be living on their own more frequently than their counterparts $(\mathrm{r}=-.18, p=.03)$, pointing to the possibility that they may have less social support. In addition, it can be speculated that over-involvement with the needs of others may push some of these 'others' away, potentially creating another source of burden.

In sum, unmitigated communion appears to be a risk factor, regardless of the levels of burden it may be associated with. The risk caregivers are subjected to by virtue of their caregiving role, therefore, may be further compounded in caregivers with high unmitigated communion. The pathways linking unmitigated communion with emotional distress, however, appear to be more complex and needs to be studied in future research. 


\section{Personality profiles}

The interactions between personality constructs created various personality profiles, some of which were associated with better well-being outcomes than others. While there was support to the hypothesis that combinations of adaptive personality constructs may lead to better well-being outcomes, the combinations of adaptive and maladaptive constructs showed mixed results.

More specifically, a profile that was created by the interaction of unmitigated communion with goal disengagement capacities, showed that participants who are involved with their caregiving duties at the expense of their own needs, experienced a larger increase in purpose in life overtime if they persisted on pursuing their goals, even if they were unattainable (see Figure 10). As was suggested earlier, the goals of caregivers with high unmitigated communion may well be related to their caregiving responsibilities and not to other personal goals: high unmitigated communion caregivers may have few goals outside of their caregiving role because they may feel guilty if they are not fully immersed in their caregiving duties. Persisting in caregiving-related goals and abandoning other goals is thus congruent with their unmitigated communion characteristic. In this regard, it can be speculated that in the absence of goals other than caregiving-related ones, the persistence among high unmitigated communion participants to attain goals that they perceive as unattainable may eventually create some purpose beyond the caregiving relationships.

Additionally, high unmitigated communion caregivers also showed smaller increases in negative affect over time if they didn't try to engage in new goals (see 
Figure 11). Congruent with the previous finding on the potential negative role of goal reengagement, this suggests that over-involved caregivers may sustain their 'sense of duty', purpose, and reduce their negative feelings by persisting in attaining their goals and not seeking new ones. It may be that it is particularly difficult for caregivers high in unmitigated communion to get stretched too thin and as a consequence to experience difficulty with caregiving interactions because caregiving is an essential part of their self-definition.

Other significant profiles consisted of the interaction between optimism and goal disengagement capacities. The cross-sectional analysis showed that pessimistic caregivers experienced high levels of depression regardless of their goal disengagement capacities, while optimists experienced less depressive symptoms especially if they could let go of unattainable goals (see Figure 7). This suggests that goal disengagement can be particularly adaptive for optimists. In this regard, it seems interesting to consider the previously discussed findings, showing that, unlike goal disengagement, optimism may not be associated with adaptive outcomes if caregivers experience particularly high levels of burden. Here, the combination of high optimism and high goal disengagement could exert a protective function and prevent highly burdened caregivers from experiencing high levels of depressive symptoms.

The longitudinal analysis further showed that optimists experienced increases in purpose over time, independently of whether they had good or poor goal disengagement capacities. However, pessimistic caregivers experienced increases in purpose in life over time only if they persisted in pursuing unattainable 
goals, but did not improve levels of purpose if they abandoned unattainable goals (see Figure 9). This finding suggests that pessimists, who may give up on goals too early because they have low outcome expectancies, may in fact attain some goals if they do persist on attaining them, and by so doing may experience higher levels of purpose in life. Interestingly, this conclusion is consistent with the previous finding that caregivers high in unmitigated communion may show better well-being if they do not give-up on goals. In both scenarios, it seems that certain individuals could construe attainable goals as unattainable: high unmitigated communion participants could feel that they need their resources for caregiving activities, and pessimists due to their negatively-biased outcome expectancies in general. In such circumstances, it may be beneficial for a person's subjective well-being if they have a difficult time abandoning goals, because they may learn over time that these goals can indeed be attained.

Finally, there was a significant cross-sectional interaction effect of goal disengagement and goal reengagement capacities on levels of negative affect. Caregivers who were able to let go of goals they deemed unattainable experienced less negative affect if they didn't try to find new goals for themselves (see Figure 8). This interaction effect suggests that negative affect may arise in the context of caregiving if individuals are either not able to disengage or engage too easily in other new goals. It is consistent with previously discussed findings and offers further support to the notion that the ability to let go of unattainable goals is an adaptive capacity, but the tendency to reengage in new goals can be either adaptive or maladaptive, depending on the circumstances. Of interest is that this was the only 
interaction that was found to be mediated by a care-specific coping behavior - selfblame. Goal disengagement capacities were instrumental in reducing caregivers' negative affect and self-blame, particularly among participants with poor goal reengagement capacities, suggesting that among caregivers with poor goal reengagement capacities, those who are able to disengage experience reduced levels of negative affect at least in part because they do not tend to blame themselves for caregiving problems.

In conclusion, in the face of caregiving burden, both adaptive and maladaptive personality constructs created profiles that were associated with participants' subjective well-being, in both the cross-sectional and longitudinal analyses. However, while there was some support for the hypotheses, there were also some surprising findings. For example, while goal disengagement was associated with higher levels of subjective well-being among optimists, the opposite effect was found among pessimists and among participants high in unmitigated communion. This pattern of findings shows that reliable interactions can be found between different personality constructs in predicting caregivers' quality of life. In addition, it suggests that these processes can be more complex than originally expected. Thus, it is not particularly surprising that only modest evidence was found for the mediating role of coping in the associations between personality profiles and subjective well-being. For example, self-blame was found to exert a marginal indirect effect on the association between goal disengagement and goal reengagement capacities on negative affect in the cross sectional analysis. Future research is needed to elucidate these complex associations and to illuminate 
pathways to quality of life among caregivers of family members with mental illness.

Contributions for theory

Overall, the study's findings have important implications for theory and research in the area of self-regulation and quality of life. First, they suggest that some individuals can thrive in the context of a chronic and severe stressor, such as caregiving for a mentally ill family member. The results showed that even in this situation, caregivers can experience high levels of subjective well-being. These findings are consistent with theory and research indicating that caregiving (e.g., Kim et al., 2007) and other goal-related problems, for example regret experiences (Bauer \& Wrosch, 2011; King \& Hicks, 2007) do not necessarily result in detrimental developmental outcomes. In addition, they illuminate some of the personality variables that can support such adaptive developments in the context of stressful caregiving. These personality variables are associated with the capacity to adjust to unattainable goals, have optimistic outcome expectancies, use effective coping strategies, or being low in unmitigated communion. These findings may inform research designed to elaborate theories on adaptive factors in the context of chronic stressors.

Second, the findings provide evidence that specific coping strategies represent a mechanism that links goal adjustment capacities and dispositional optimism with indicators of subjective well-being. While such effects have been previously reported to explain the influence of optimism (Scheier et al., 1989), this research documents that similar pathways can be identified for a person's capacity to adjust to unattainable goals. Overall, this implies that optimism and goal 
adjustment may not exert only direct emotional benefits. Instead, their effects extend to facilitating adjustment to stressors that impose constraints on other desired goals. These findings further suggest that to understand the process of successful adjustment to difficult life circumstances more comprehensively, it is important to consider both, how individuals tend to react to problems in general and how they regulate specific stressors. While these different levels of personality functioning have been clearly articulated in personality theory, the associations between levels are not always conceptualized (McAdams \& Olson, 2010). In this regard, the theoretical model used in this research assumes that individual differences in general self-regulation tendencies unfold their adaptive value by influencing specific self-regulation behaviors. In support of this idea, the research findings showed that goal adjustment capacities and optimism can predict how a person copes with specific stressors and thereby affect the person's subjective well-being.

Third, the study's results suggest two different mechanisms that can explain how personality factors and caregiver burden work together to influence subjective well-being. First, the effects of adaptive personality factors on subjective well-being can be mediated by low levels of caregiver burden. Second, adaptive personality factors can buffer the adverse consequences of burden on a person's general subjective well-being. This implies that there are, at least, two different functions of individual differences in personality, which are not mutually exclusive. They may reduce the burden associated with a difficult situation and thereby directly improve quality of life. In addition, among those individuals who are particularly burdened by specific life circumstances, they may prevent spillover effects on general 
indicators of quality of life, such as depressive symptomatology.

Additionally, the findings document how general personality factors can have different effects on specific versus general indicators of subjective well-being. In particular, individuals' capacities to identify and pursue new goals were shown to be associated with high levels of purpose and positive affect, but also with high levels of caregiving burden. This implies that there may be a trade-off that comes with reengaging in new goals. As a result, research needs to assess situation-specific and general indicators of subjective well-being to avoid a misinterpretation of the effects of personality factors on adjustment to critical life circumstances. In addition, these findings raise the question whether it is more beneficial for a person's long-term quality of life to avoid stress-specific burden or to ensure that life continues with purpose. While this question can be empirically determined only in long-term follow-ups, it is suggested that the experience of general subjective well-being, such as purpose, may ultimately serve more critical adaptive functions, as it represents a motivational resource necessary for creating long-term developmental benefits across different areas of life (for primacy of primary control capacity, see Heckhausen et al., 2010).

The findings also suggest that personality factors may serve different functions in high versus low burden situations. For example, optimism was associated with subjective well-being especially when caregiving burden was low. When burden was high, optimism appeared to loose some of its luster and was not sufficient in predicting good well-being. In such situations, additional adaptive tendencies may be required, such as high levels goal adjustment capacities. This 
may imply that there are different pathways that lead to high subjective well-being in stressful circumstances. First, when stress-related burden is not extremely high, personality factors that support that attainment of goals can reveal their adaptive effects; supposedly by promoting progress with important and attainable goals. Second, in high-burden situations, individuals need to be able to let go in order to redirect scarce resources to the management of stressful situations.

Finally, the results point to the need of more complex theories that incorporate different personality factors, and their interactions. For example, the capacity to disengage from unattainable goals was shown to benefit some individuals (e.g., optimists), but was detrimental for others (e.g., pessimistic, or high unmitigated communion participants). This implies that complex interactions between different personality variables are likely to exert influence on indicators of subjective well-being, rather than simple main effects of single personality constructs. Thus, more research is needed to conceptualize and test these interactions between different personality variables in the management of stressful life circumstances. Such research could be instrumental for better understanding how the quality of life of high burdened caregivers can be sustained and improved.

\section{Implications for practice}

The relevance of this study's findings extends beyond its implications for theory. It can be used to make recommendations that could ultimately affect caregivers, as well as their ill relatives. Family caregivers play an important role in the trajectory of mental illness: they can facilitate - but they can also get in the way of a loved one's journey of recovery. Family caregivers have a central role in 
integrating the emerging recovery vision as they cope with mental illness in a loved one.

For many years the treatment of mental illness was guided by a medical model (Adler, 1981; Harding \& Zahniser, 1994). In the absence of a known cure, controlling the illness symptoms traditionally included mainly medical treatment. However, a gradual shift in the perception of mental illness has been translated into a growing repertoire of treatment options (Harding \& Zahniser, 1994). Viewing mental illness as a condition that subjects the afflicted to a life of doom and gloom is gradually changing: people with mental illness are increasingly being viewed as individuals with challenges, much like other persons with physical or other conditions (Davidson, O'Connell, Tondora, Staeheli, \& Evans, 2005). Within this paradigm shift, the vision of recovery is a central tenet. Contrary to earlier outlooks, this view posits that recovery from mental illness does not require the remission of symptoms or of other deficits brought on by the disorder. Rather, recovery involves the incorporation of one's illness with a sense of hopefulness about the future, and particularly about one's ability to build a positive sense of self and social identity despite continuing to have mental illness (Davidson et al., 2005). The goal is not to lead the person back to a pre-existing state of health, but rather to recognize that recovery is ongoing or lifelong in nature.

Like people with mental illness, caregivers have been victimized by the stigma associated with mental illness. Stigma is often considered more debilitating than the illness itself, and often leads to internalization, resulting in self-stigma (Kirby \& Keon, 2006). The potentially negative influence families may have on the 
recovery of an ill relative is reflected in the findings of a study that explored the relationships between stigma, self-concept, and recovery (Markowitz, Angell, \& Greenberg, 2011). This study demonstrated that how mothers perceive their ill relative is associated with the relative's levels of symptoms, self-efficacy and quality of life. These findings are also consistent with earlier studies that found robust associations between caregiver criticism ("expressed emotions") and symptomatic behavior (Renshaw, 2008). Both findings reinforce the notion that management of mental illness is more than symptom control, as is often indicated by a narrowly defined medical model, and that, at least in part, it is a socialpsychological process: the ways people think about individuals with mental illness affect the beliefs and actions of those with mental illness and influence the trajectory of their illness and recovery (Markowitz et al., 2011).

To be able to help, rather than hinder, many caregivers need first to revise their own perception of the illness, which is often informed by societal and selfstigma, then adjust their relationships with the ill relative. The ability to do that would likely have an important impact on the well-being of both the ill individual and the caregiver. While personality is not easily modifiable, the coping behaviors that may be associated with some personality constructs suggest that some interventions could help to strengthen effective care-specific coping, or minimize non-effective coping behaviors. For example, encouraging the reduction of selfblame; encouraging the acceptance of situations they have no control over, or enhancing the ability to reframe stressful situations in positive terms, all can be instrumental in facilitating a recovery process for the ill relative, and in protecting 
the integrity of the caregiver's own well-being.

Blaming parents (especially mothers) for mental illness in a relative, has been common during the better part of the twentieth century and was fueled by theories such as the 'schizophrenogenic mother' (Fromm-Reichmann, 1948). That today parents still continue to blame themselves for their relative's illness, is a testimony to the internalization of that blaming attitude. Undoing self-blame could be an important intervention aimed at assisting caregivers to establish more constructive relations with ill relatives and consequently affect their own wellbeing.

Infrequent use of self-blame or substances were found to be facilitated by high levels of goal disengagement capacities, and led to smaller increases in depressive symptoms and negative affect over time in this study. This suggest that encouraging caregivers to engage in such behaviors my have an impact on their self-regulating capacity and consequently on well-being outcomes.

The ability to engage in new goals was found to facilitate the use of positive reframing and was associated with the reduction of caregivers' depressive symptomathology. Integrating a recovery vision could help caregivers modify their view of the illness, adjust their goals accordingly and potentially lead to a decrease in the negative effects associated with caregiving.

Self-blame was also associated with pessimism and led to higher levels of negative affect. While in this study pessimism facilitated self-blaming behavior, the possibility of two-way relations between personality constructs and coping behaviors suggests that the reduction of self-blame could also tone down the 
negative effects of pessimism. Acceptance, on the other hand, was associated with a decrease in depressive symptomathology, pointing to the importance of encouraging acceptance as an effective coping behavior in circumstances on which the caregiver has no control.

Goal reengagement capacities in this study were associated with both positive and negative outcomes. These findings suggest that while caregivers may consider seeking new goals to replace unattainable goals in order to sustain a sense of purpose in living, they should also be cautious not to stretch themselves too thin in a way that could interfere with their caregiving role. Professional guidance could help caregivers establish a healthy balance between caregiving for their relative and maintaining a certain level of diversity (Linville, 1987).

Additional findings point to the importance of offering information, education and support to caregivers as early as possible. Younger caregivers showed higher levels of depressive symptoms than older caregivers. Moreover, recent diagnosis was associated with larger increases in depressive symptoms over time, suggesting that the less time caregivers deal with the illness the more depressed they appear to be. Since mental illness typically starts in late adolescence or early adulthood, it is likely that recent diagnosis corresponds to younger age in caregivers. The two findings together reinforce the importance of early supports to caregivers.

The findings also link married or cohabiting participants with higher purpose in life, lower burden, lower levels of depressive symptoms and smaller increases in depressive symptoms over time. Since unmitigated communion individuals were 
found to live alone more frequently than other caregivers, it is possible that unmitigated communion may precipitate consequences such as living alone, experiencing more burden and possibly, less support. Helping unmitigated communion caregivers 'ease up' on this tendency, therefore, could mitigate some of the negative consequences and possibly affect caregiver's well-being positively.

The involvement of family members with the caring for an ill family member is generally viewed as potentially beneficial for both care recipient and caregiver. However, to maximize these benefits and to minimize the potentially negative consequences, it is important to support caregivers in their adaptation to stressful situations. Normative stressors, as well as transitions and strains, call upon families to use their internal resources in order to adjust to such stressful situations. However, non-normative stressors - such as mental illness of a family member require both internal and external resources in order to adapt to the stressors (McCubbin \& McCubbin, 1988). Families vary in their inherent resilience and adaptation capacities; proper interventions can foster resilience and enhance effective coping.

Finally, well-being outcomes appear to be mediated by factors such as meaning in caregiving, and emotional support (Noonan \& Tennstedt, 1997). Moreover, caregivers' subjective perception of their difficulties appear to predict well-being more than the objective characteristics of their circumstances. This suggests that interventions in support of caregivers should consider not only the practical consequences of caregiving, but also the personal meaning associated with caregiving. 


\section{Limitations and Future Research}

There are limitations to this study that need to be addressed in future research. First, the analytical approach used in this study examined how personality traits are associated with levels of coping rather than with changes in coping over time. This approach was chosen because of the chronic nature of the stressor in this study. In fact, participants cared on average almost 16 years for their relatives, which makes it rather unlikely that new coping strategies would be adopted over a comparably short period of time. However, future research should extend this analysis by studying adjustment to recent and acute stressors (e.g., development of a new physical health problem). In such circumstances, it would be expected that personality constructs such as goal adjustment capacities and optimism would predict changes in coping over time and thereby influence quality of life.

Second, the results showed that the effects of goal adjustment capacities and optimism on subjective well-being were mediated mostly by emotion-focused coping, and mainly by the avoidance of negative coping behaviors. These patterns may be specific to the stressor considered in this study. Caring for a family member with mental illness is a chronic stressor that is likely to persist in participants' lives for many years. Moreover, because the patients and their doctors possess much control over the course of the illness, a caregiver's efforts at active coping may, at times, be unsuccessful or only partially successful. In fact, these results are consistent with earlier studies that noted that problem-solving strategies are likely to be useful when the situation can be changed, but not when a difficult situation cannot be changed. In such cases, problem-solving efforts may be not only 
ineffective but can further exacerbate the caregiver's stress (Stengard, 2002). It appears, therefore, that the adaptive value of coping strategies depends in part on the situational circumstances.

It will not be surprising, therefore, if active coping plays an important role in circumstances in which the stressor can be overcome or eliminated more easily, as in education or work-related problems. Future research should therefore investigate a wider range of stressful life circumstances in order to establish if and when coping strategies that are functionally associated with goal adjustment capacities and optimism are context dependent.

It is important to note, however, that while no active coping behaviors were found to mediate the associations between personality constructs and well-being, the participants in this study have already demonstrated active coping by virtue of their involvement with a family association. Such support seeking behavior could be the equivalent of seeking instrumental or emotional support, as well as planning, in order to cope with caregiving responsibilities.

Third, this study focused on subjective well-being as an indicator of successful adaptation. In this regard, it seems important to note that some research has suggested that adaptive development may require individuals to confront and accept adverse life circumstances, which could compromise subjective well-being in the short-run, but contribute to adaptive personality development over a longer period of time (e.g., Helson \& Roberts, 1994; King \& Hicks, 2007). To address this possibility, it is suggested that future research should examine a wider range of 
indicators of successful adaptation, such as psychological well-being or ego development (Loevinger, 1976; Ryff, 1989b) over a longer period time.

Further research could also explore the different types of goals caregivers may have. While goal adjustment capacities are defined broadly, this concept is likely to be understood in relation to one's own goals for self. A statement such as "I stay committed to a goal for a long time" or "I think about other goals to pursue", are likely to imply goals an individual identifies for self. However, in the context of caregiving to a mentally ill relative, there may be more than the typically perceived goals for oneself. These additional goals include goals the caregivers may have for their ill relative, as well as goals related to the way they perceive their caregiving roles. While these may not be readily articulated as goals, it is suggested that they are indeed goals, whose attainment or lack thereof may have a similar impact on one's self regulating process and the resultant well-being.

Caregivers' goals for an ill relative are often perceived as hopes or wishes, such as "I wish my son would acquire higher education so I am encouraging him to go to university." If not congruent with the circumstances, such as relative is too ill to pursue higher education, persisting on such a goal may be akin to a poor goal disengagement capacity. On the other hand a statement such as "she can still have a good life even if she cannot be a doctor or a lawyer," may demonstrate an ability to disengage from an unattainable goal for the ill relative.

Caregiving related goals and the associated behaviors are often associated with the way caregivers perceive their caregiving roles vis-à-vis the ill relative. Here, statements such as "I will reduce my telephone contact with her to once a 
day," or "I will stop nagging him about his medications", may represent a desire to adjust, or regulate goal attainment. On the other hand caregivers who attempt to assume ongoing and full control over their relatives' behaviors may well be compromising their relative's opportunity to take responsibility for themselves, and at the same time compromise their own well-being. Future research could explore the latter two types of goals by referring to them explicitly, in order to determine their potentially distinct characteristics and their associations with subjective wellbeing.

A source of burden that was not addressed in the current study may be associated with the relationship of caregivers with the health-care system. Many health care professionals still exclude families from the treatment process, possibly because of a lingering perception that families in which a member is diagnosed with mental illness are dysfunctional and may interfere with the treatment process. The burden experienced by family caregivers, therefore, may be related, in part, to that exclusion, which is contrary to many families' desire to be included in the treatment process. Additionally, the needs of the family, which are related to both the caregiving role and to their status as 'secondary victims' of mental illness, are seldom addressed by professionals. This common lack of support is likely to compound the burden caregivers already face by virtue of their caregiving activities and the emotional toll mental illness takes. This study explored the burden directly associated with the caregiving role, but not the burden that may be associated with the perception of professionals and the lack of support. In order to adjust practices to address the entire burden experience, future research need to further explore the 
burden components that include the perception of professionals and the availability of support.

Findings from other studies that link the ability to disengage from unattainable goals with an amelioration of the effect of normative health problems on older adults' depressive symptoms (Dunne et al, 2011), may be relevant in this context and could encourage further research with elderly caregivers as the target population. If health problems can trigger depressive symptomathology in older adults who are not caregivers, the impact of such problems on elderly caregivers may be even bigger: they are likely to be preoccupied not only with attaining their own goals, but also with performing their caregiving goals. This may create a downward spiral where burden leads to health problems and consequently to depression. The ability, therefore, to disengage from unattainable goals in both contexts may become even more vital as a protection for the caregiver's subjective well-being. Thus, future research should incorporate a comprehensive assessment of caregivers' physical health to examine these possibilities.

Additionally, studying meaning in caregiving and its possible association with other personality constructs could shed additional light on the influence personality constructs may exert on subjective well-being. Earlier studies found that meaning in caregiving is negatively associated with depressive symptoms and positively associated with self-esteem (Noonan \& Tennstedt, 1997). However, it was not found to be associated with caregiving overload: while some caregivers who have experienced high burden reported gaining meaning from their caregiving role, others did not. 
While an association between meaning and optimism may be more readily expected, the potential association between meaning and unmitigated communion, for example, is less clear. This study's finding that unmitigated communion can be associated with maintaining a sense of purpose and reduce negative affect if caregivers persist on attaining their goals and do not attempt to find alternative goals suggests that high unmitigated communion caregivers may attach meaning to their caregiving goals. These findings point to the importance of studying caregiving within a multivariate model. For example, overload can be understood as a primary stressor variable, or alternatively as a mediating variable that serves to weaken the relationship between stressors and outcomes, or an appraisal variable (Lawton, Moss, Kleban, Glicksman, \& Rovine, 1991). These possibilities illustrate the complexity of the caregiving experience and the need for further research.

It is important to note that the sample used in this study consisted of caregivers for a relative with mental illness who are also members of a family association. By virtue of such affiliation, which offers help and support to caregivers, and as mentioned earlier, these caregivers have demonstrated active help-seeking behavior. The consequences of the caregiving experience for caregivers who do not seek active support from a family association may be different; if they don't seek help elsewhere, they may feel even more burdened. The conclusions of this study, therefore, may under-estimate the burden many caregivers are subjected to and its impact on their subjective well-being. Further research with caregivers who are not members of family associations is needed, to allow for the generalization of the conclusions. 
In a related vein, all the participants in the study can be described as 'engaged' caregivers who are involved with their ill relatives in one way or another. However, there are families who, for different reasons, become disengaged from their ill relatives. It would be important to explore the reasons for disengagement and the consequences for these caregivers' subjective well-being. A sense of isolation and despair may stem from lack of early and adequate support, and may result in disengagement from caregiving duties; personality tendencies may contribute as well. Further investigation of personality traits in the context of caregiving to a mentally ill relative could help to better understand why some caregivers chose to abandon their caregiving responsibilities.

Additionally, because of the commonly long-term nature of caregiving in the context of mental illness, caring for a mentally ill adult child, for example, may at times coincide with caring for an elderly parent. This dual role may exacerbate the stress associated with caregiving and further influence the caregiver's subjective well-being. This scenario was not explored in this study and future research could explore it further and compare the outcomes of 'compound' caregiving with caregiving in one context only.

As noted earlier, there are three principal players in the context of mental illness: care-recipients, unpaid caregivers and professional care providers. The effects of mental illness can have far-reaching effects on the entire family, including care recipient and caregivers, as well as on care providers and the healthcare system at large; and the consequences for public health cannot be under-estimated. This thesis, however, focused on the experience of caregivers only, with special attention 
to the impact of mental illness on their subjective well-being. Further research could study the three principal players together (care-recipients, caregivers and care providers) and shed light on the interrelations, consequences and potential recommendation for improvements.

In this study, $p$-value $<.05$ was considered significant because the study's hypotheses were theory based. However, given that there are differences in opinion regarding alpha level correction for multiple comparisons (e.g., Perneger, 1998), the results should nonetheless be interpreted with caution. Stringent analyses in future research would be necessary in order to confirm the trends found in this study and further explore the various associations. In addition, it would be useful to replicate the study's results with a larger and more heterogeneous sample.

Finally, it is of interest to note that the study participants experienced high levels of caregiver burden, which places them at the $75^{\text {th }}$ percentile of burden typically experienced by dementia caregivers (Hébert, Bravo, \& Preville, 2000). Such elevated levels of burden could put some study participants at risk of developing patterns of biological dysregulation and subsequent physical health problems (Cohen, Janicki-Deverts, \& Miller, 2007). It is therefore suggested that future research should conduct long-term longitudinal studies to examine how personality factors, coping, and associated subjective well-being can predict biomedical outcomes and protect the quality of life of individuals who experience chronically stressful life circumstances. 


\section{References}

Abramson, L. Y., Seligman, M. E., \& Teasdale, J. D. (1978). Learned helplessness in humans: Critique and reformulation. Journal of Abnormal Psychology, 87, 49-74.

Adler, D. A. (1981). The medical model and psychiatry's tasks. Hospital and Community Psychiatry, 32, 387-392.

Aiken, L. S., \& West, S. G. (1991). Multiple regression: Testing and interpreting interactions. Newbury Park, CA: Sage.

Aldwin, C. M., \& Revenson, T. A. (1987). Does coping help? A reexamination of the relation between coping and mental health. Journal of Personality and Social Psychology, 53, 337-348.

Alloy, L. B., Abramson, L. Y., Whitehouse, W. G., Hogan, M. E., Panzarella, C., \& Rose, D. T. (2006). Prospective incidence of first onsets and recurrences of depression in individuals at high and low cognitive risk for depression. Journal of Abnormal Psychology, 115, 145-156.

Allport, G. W. (1955). Becoming. New Haven, CT: Yale University Press.

Allport, G. W. (1960). The open system in personality theory. Journal of Abnormal Social Psychology, 61, 301-311.

Allport, G. W. (1961). Pattern and growth in personality. New York: Holt, Rinehart \& Winston.

Allport, G. W. (1966). Traits revisited. American Psychologist, 21, 1-10.

Andrews, F. M., \& Whitey, S. B. (1976). Social indicators of well-being: Americans' perception of life quality. New York: Plenum Press. 
Ayres, L. (2000). Narratives of family caregiving: The process of making meaning. Research in Nursing \& Health, 23, 424-434.

Bakan, D. (1966). The duality of human existence. Chicago: Rand McNally.

Baronet, A. M. (1999). Factors associated with caregiver burden in mental illness: A critical review of the research literature. Clinical Psychology Review, 19, 819-841.

Batson, C. D. (1995). Prosocial motivation: Why do we help others? In A. Tesser (Ed.), Advanced social psychology (pp. 333-381). New York: McGraw-Hill.

Bauer, I. (2004). Unattainable goals across adulthood and old age: Benefits of goal adjustment capacities on well-being. Concordia University, Montreal, Quebec, Canada.

Bauer, I., \& Wrosch, C. (2011). Making up for lost opportunities: The protective role of downward social comparisons for coping with regrets across adulthood. Personality and Social Psychology Bulletin, 37, 215-228.

Baumeister, R. F., Bratslavsky, E., Muraven, M., \& Tice, D. M. (1998). Ego depletion: Is the active self a limited resource? Journal of Personality and Social Psychology, 74, 1252-1265.

Beck, A. T. (1967). Depression, clinical, experimental, \& theoretical aspects. Hober Medical Division, Harper \& Row, New York.

Billings, A. G., \& Moos, R. H. (1984). Coping, stress, and social resources among adults with unipolar depression. Journal of Personality and Social Psychology, 46, 877-891. 
Bolger, N. (1990). Coping as a personality process: A prospective study. Journal of Personality and Social Psychology, 59, 525-537.

Bolger, N., \& Zuckerman, A. (1995). A framework for studying personality in the stress process. Journal of Personality and Social Psychology, 69, 890-902. Borthwick-Duffy, S. A. (1992). Quality of life and quality of care in mental retardation. In L. Rowitz (Ed), Mental retardation in the year 2000 (pp. 5266). Berlin: Springer-Verlag.

Bradburn, N. M. (1969). The structure of psychological well-being. Chicago: Aldine.

Brissette, I., Scheier, M. F., \& Carver, C. S. (2002). The role of optimism in social network development, coping, and psychological adjustment during a life transition. Journal of Personality and Social Psychology, 82, 102-111.

Carter, P. A., \& Chang, B. L. (2000). Sleep and depression in cancer caregivers. Cancer Nursing, 23, 410-415.

Carver, C. S. (1997). You want to measure coping but your protocol's too long: Consider the brief COPE. International Journal of Behavioral Medicine, 4, 92-100.

Carver, C. S., \& Gaines, J. G. (1987). Optimism, passimism and post-partum depression. Cognitive Therapy and Research, 11, 449-462.

Carver, C. S., \& Scheier, M. F. (1981). Attention and self-regulation: A control theory approach to human behavior. New York, NY: Springer Verlag.

Carver, C. S., \& Scheier, M. F. (1990). Origins and functions of positive and negative affect: A control process view. Psychological Review, 97, 19-35. 
Carver, C. S., \& Scheier, M. F. (1994). Situational coping and coping dispositions in a stressful transaction. Journal of Personality and Social Psychology, 66, 184-195.

Carver, C. S., \& Scheier, M. F. (1998). On the self-regualtion of behavior. New York: Cambridge University Press.

Carver, C. S., Scheier, M. F., \& Segerstrom, S. C. (2010). Optimism. Clinical Psychology Review, 30, 879-889.

Carver, C. S., Scheier, M. F., \& Weintraub, J. K. (1989). Assessing coping strategies: A theoretically based approach. Journal of Personality and Social Psychology, 56, 267-283.

Chang, E. C. (1998). Dispositional optimism and primary and secondary appraisal of a stressor: Controlling for confounding influences and relations to coping and psychological and physical adjustment. Journal of Personality and Social Psychology, 74, 1109-1120.

Chappell, N. L. \& Reid, R. C. (2002). Burden and well-being among caregivers: Examining the distinction. The Gerontologist, 42, 772-780.

Charney, D. S. (2004). Psychobiological mechanisms of resilience and vulnerability: Implications for successful adaptation to extreme stress. American Journal of Psychiatry, 161, 195-216.

Christensen, K. A., Stephens, M. A., \& Townsend, A. L. (1998). Mastery in women's multiple roles and well-being: Adult daughters providing care to impaired parents. Health Psychology, 17, 163-171. 
Clark, R. E., \& Drake, R. E. (1994). Expenditures of time and money by families of people with severe mental illness and substance use disorders. Community Mental Health Journal, 30, 145-163.

Clark, R. E., Teague, G. B., Ricketts, S. K., Bush, P. W., Keller, A. M., Zubkoff, M., et al. (1994). Measuring resource use in economic evaluations: Determining the social costs of mental illness. Journal of Mental Health Administration, 21, 32-41.

Cohen, S., Janicki-Deverts, D., \& Miller, G. E. (2007). Psychological stress and disease. Journal of American Medical Association , 298, 1685-1687.

Costa, P. T., \& McCrae, R. R. (1997). Stability and change in personality assessment: The revised NEO Personality Inventory in the year 2000. Journal of Personality Assessment, 68, 86-94.

Costa, P. T., \& McCrae, R. R. (1980). Still stable after all these years: Personality as a key to some issues in adulthood and old age. In P. B. Baltes \& O. G. Brim Jr. (Eds.), Life span development and behavior (pp.65-102). New York: Academic.

Costa, P. T., \& McCrae, R. R. (1985). The NEO Personality Inventory manual. Odessa, FL: Psychological Assessment Resources.

Culver, J. L., Arena, P. L., Antoni, M. H., \& Carver, C. S. (2002). Coping and distress among women under treatment for early stage breast cancer: Comparing African Americans, Hispanics and non-Hispanic whites. Psychooncology, 11, 495-504. 
Cummins, R. A. (1996). The domain of life satisfaction: An attempt to order chaos. Social Indicators Research, 38, 303-332.

Davidson, L., O'Connell, M. J., Tondora, J., Staeheli, M. R., \& Evans, A. C. (2005). Recovery in serious mental illness: A new wine or just a new bottle? Professional Psychology: Research and Practice, 36, 480-487.

DeWall, C. N., Baumeister, R. F., Stillman, T. F., \& Gailliot, M. T. (2007). Violence restrained: Effects of self regulation and its depletion on aggression. Journal of Experimental and Social Psychology, 43, 62-76.

Diener, E., Emmons, R. A., Larsen, R. J., \& Griffin, S. (1985). The Satisfaction With Life Scale. Journal of Personality Assessment, 49, 71-75.

Dunn, D. S. (1996). Well-being following amputation: Salutary effect of positive mean, optimism and control. Rehabilitation Psychology, 41, 285-302.

Dunne, E., \& Wrosch, C. (2009, July). The longitudinal associations between functional disabilities, goal adjustment capacities and depressive symptoms in older adults. Poster presented at the 19th IAGG World Congress of Gerontology and Geriatrics, Paris, France.

Dunne, E., Wrosch, C., \& Miller, G. E. (2011). Goal disengagement, functional disability, and depressive symptoms in old age. Health Psychology. Advance online publication. doi.10.1037/a0024019

Dyck, D. G., Short, R., \& Vitaliano, P. P. (1999). Predictors of burden and infectious illness in schizophrenia caregivers. Psychosomatic Medicine, 61, 411-419. 
Emmons, R. A. (1986). Personal strivings: An approach to personality and subjective well-being. Journal of Personality and Social Psychology, 51, 1058-1068.

Family Caregiver Alliance (2007). Family caregiving: State of the art, future trends. Report from a National Conference. San Francisco: Author.

Fischer, R., \& Boer, D. (2011). What is more important for national well-being: Money or autonomy? A meta-analysis of well-being, burnout, and anxiety across 63 societies. Journal of Personality and Social Psychology, 101, 164184.

Folkman, S. (1997). Positive psychological states and coping with severe stress. Social Science and Medicine, 45, 1207-1221.

Folkman, S., \& Lazarus, R. S. (1980). An analysis of coping in a middle-aged community sample. Journal of Health and Social Behavior, 21, 219-239.

Folkman, S., \& Lazarus, R. S. (1985). If it changes it must be a process: Study of emotion and coping during three stages of a college examination. Journal of Personality and Social Psychology, 48, 150-170.

Folkman, S., \& Lazarus, R. S. (1988). Coping as a mediator of emotion. Journal of Personality and Social Psychology, 54, 466-475.

Folkman, S., \& Moskowitz, J. T. (2000). Positive affect and the other side of coping. American Psychologist, 55, 647-654.

Fritz, H. L., \& Helgeson, V. S. (1998). Distinctions of unmitigated communion from communion: Self-neglect and overinvolvement with others. Journal of Personality and Social Psychology, 75, 121-140. 
Fromm-Reichmann, F. (1948). Notes on the development of treatment of schizophrenics by psychoanalytic psychotherapy. Psychiatry, 11(3), 263-273.

Gallant, M. P. \& Connell, C. M. (1998). The stress process among dementia spouse caregivers: Are caregivers at risk for negative health behavior change? Research on Aging, 20, 267-297.

Given, C. W., Stommel, M., Given, B., Osuch, J., Kurtz, M. E., \& Kurtz, J. C. (1993). The influence of cancer patients' symptoms and functional states on patients' depression and family caregivers' reaction and depression. Health Psychology, 12, 277-285.

Gottlieb, B. H., \& Rooney, J. A. (2004). Coping effectiveness: Determinants and relevance to the mental health and affect of family caregivers of persons with dementia. Aging and Mental Health, 8, 364-373.

Gravitz, H. L. (2000). The binds that tie: Families under the influence of mental illness. The Journal of NAMI California, 11, 46-48.

Gravitz, H. L. (2004). Mental illness and the family: Unlocking the doors to triumph. Santa Barbara, CA: Healing Visions Press.

Grunfeld, E., Coyle, D., Whelan, T., Clinch, J., Reyno, L., Earle, C. C., et al. (2004). Family caregiver burden: Results of a longitudinal study of breast cancer patients and their principal caregivers. Canadian Medical Association Journal, 170, 1795-1801.

Harding, C. M., \& Zahniser, J. (1994). Empirical correction of seven myths about schizophrenia with implications for treatment. Acta Psychiatrica Scandinavica, suppl. 384, 140-146. 
Hasin, D. S., \& Grant, B. F. (2002). Major depression in 6050 former drinkers: Association with past alcohol dependence. Archives of General Psychiatry, 59, 794-800.

Hatfield, A. B. (1983). What families want of family therapists. In W. McFarlane (Ed.), Family Therapy in Schizophrenia. New Yourk: Guilford Press.

Hatfield, A. B., \& Lefley, H. E. (1987). Families of the mentally ill. New York: Guilford Press.

Health Canada (2002), National Profile of Family Caregivers in Canada - Final Report. Prepared by Decima Research Inc. www.hc-sc.gc.ca.

Hébert, R., Bravo, G., \& Preville, M. (2000). Reliability, validity and reference values of the Zarit Burden Interview for assessing informal caregivers of community dwelling older persons with dimentia. Canadian Journal on Aging, 19, 494-507.

Heckhausen, H. (1991). Motivation and action. New York, NY: Springer-Verlag.

Heckhausen, J., Wrosch, C., \& Fleeson, W. (2001). Developmental regulation before and after a developmental deadline: The sample case of "biological clock" for childbearing. Psychology and Aging, 16, 400-413.

Heckhausen, J., Wrosch, C., \& Schulz, R. (2010). A motivational theory of lifespan development. Psychological Review, 117, 32-60.

Helgeson, V. S., \& Fritz, H. L. (1996). Implications of unmitigated communion and communion for adolescent adjustment to Type I diabetes. Women's Health: Research on Gender, Behavior, and Policy, 2, 163-188. 
Helgeson, V. S., \& Fritz, H. L. (1998). A theory of unmitigated communion. Personality and Social Psychological Review, 2, 173-183.

Helgeson, V. S., \& Fritz, H. L. (1999). Unmitigated agency and unmitigated communion: Distinction from agency and communion. Journal of Research in Personality, 33, 131-158.

Helgeson, V. S., \& Fritz, H. L. (2000). The implications of unmitigated agency and unmitigated communion for domains of problem behavior. Journal of Personality and Social Psychology, 68, 1031-1057.

Helson, H. (1964). Adaptation-level theory. New York: Harper \& Row.

Helson, R., \& Roberts, B. W. (1994). Ego development and personality change in adulthood. Journal of Personality and Social Psychology, 66, 911-920.

Hollander, E. P. (1967). Principles and methods of social psychology. London: Oxford University Press.

Hulbert, N. J., \& Morrison, V. L. (2006). A preliminary study into stress in palliative care: Optimism, self-efficacy and social support. Psychology and Health Medicine, 11, 246-254.

Hunt, J. M. (1965). Traditional personality theory in the light of recent evidence. American Scientist, 53, 80-96.

Inglehart, R. (1997). Modernization and postmodernization: Cultural, economic and political change in 43 societies. Princton, NJ: Princton University Press. Inglehart, R. , Foa, R., Peterson, C., \& Welzel, C. (2008). Development, freedom, and rising happiness. Perspectives on Psychological Science, 3, 264-285. 
Keyes, C. L. (2002). The mental health continuum: From languishing to flourishing in life. Journal of Health and Social Behavior, 43, 207-222.

Khoo, S., \& Bishop, G. D. (1997). Stress and optimism: Relationships to coping and well-being. Psychologia: An International Journal of Psychology in the Orient, 40, 29-40.

Kiecolt-Glaser, J. K., Preacher, K. J., MacCallum, R. C., Atkinson, C., Malarkey, W. B., \& Glaser, R. (2003). Chronic stress and age-related increases in the proinflammatory cytokine IL-6. Proceedings of the National Academy of Sciences of the United States of America - Physical Sciences, 100, 9090-9095.

Kim, Y., Schulz, R., \& Carver, C. S. (2007). Benefit-finding in the cancer caregiving experience. Psychosomatic Medicine, 69, 283-291.

King, K. B., Rowe, M. A., Kimble, L. P., \& Zerwic, J. J. (1998). Optimism, coping, and long-term recovery from coronary artery by-pass in women. Research in Nursing \& Health, 21, 15-26.

King, L. A., \& Hicks, J. A. (2007). Whatever happened to "What might have been"? Regrets, happiness, and maturity. American Psychologist, 62, 625-636.

Kirby, M. J. L., \& Keon, W. J. (2006). Out of the shadows at last: Highlights and recommendations. Final Report of The Standing Senate Committee on Social Affairs, Science and Technology, Ottawa, Canada.

Klaaren, K. J., Hodges, S. D., \& Wilson, T. D. (1994). The role of affective expectations in subjective experience and decision-making. Social Cognition, $12,77-101$. 
Lawton, M. P. (1975). The Philadelphia geriatric center morale scale: A revision. Journal of Geronology, 30, 85-89.

Lawton, M. P., Moss, M., Kleban, M. H., Glicksman, A., \& Rovine, M. (1991). A two-factor model of caregiving appraisal and psychological well-being. Journals of Gerontology, 46, 181-189.

Lazarus, R. S. (1966). Psychological stress and the coping process. New York: McGraw-Hill.

Lazarus, R. S. (1981). The stress and coping paradigm. In Eisdorfer, D. Cohen, A. Kleinman, \& P. Maxim (Eds.), Models of clinical psychopathology (pp. 177214). New York: Spectrum.

Lazarus, R. S., \& Folkman, S. (1984). Stress, appraisal, and coping. New York: Springer.

Lefley, H. P. (1987). Aging parents as caregivers of mentally ill adult children: An emerging social problem. Hospital and Community Psychiatry, 38, 10631070.

Lefley, H. P. (1996). Family caregiving in mental illness. Thousand Oaks, CA: Sage.

Lenney, E. (1977). Women's self confidence in achievement settings. Psychological Bulletin, 84, 1-13.

Linton, R. (1945). The cultural background of personality. New York: AppletonCentury-Crofts. 
Linville, P. W . (1987). Self-complexity as a cognitive buffer against stress-related illness and depression. Journal of Personality and Social Psychology, 52, 663-676.

Loevinger, J. (1976). Ego development: Conceptions and theories. San Francisco, CA: Jossey-Bass.

Lorant, V., Deliege, D., Eaton, W., Robert, A., Philippot, P., \& Ansseau, M. (2003). Socioeconomic inequalities in depression: A meta-analysis. American Journal of Epidemiology, 157, 98-112.

Lucas, R. E., Diener, E. \& Suh, E. (1996), Discriminant validity of well-being measures. Journal of Personality and Social Psychology, 71, 616-628.

Lyons, K. S., Stewart, B. J., Archbold, P. G., Carter, J. H., \& Perrin, N. A. (2004). Pessimism and optimism as early warning signs for compromised health for caregivers of patients with Parkinson's disease. Nursing Research, 53, 354362.

Magliano, L., Fadden, G., Economou, M., Held, T., Xavier, M., Guarneri, M., et al. (2000). Family burden and coping strategies in schizophrenia: 1-year followup data from the BIOMED I study. Social Psychiatry and Psychiatric Epidemiology, 35, 109-115.

Manderscheid, R. W., \& Sonnenschein, M. A. (1996). Mental Health, United States. U.S. department of health and human services. Rockville, Maryland 20857. 
Markowitz, F. E., Angell, B., \& Greenberg, J. (2011). Stigma, reflected appraisals, and recovery outcomes in mental illness. Social Psychology Quarterly, 74, 144-165.

Marks, N. F. (1998). Does it hurt to care? Caregiving, work-family conflict, and mid-life well-being. Journal of Marriage and Family 60, 951-966.

Marsh, D. T. (1998). Serious mental illness and the family: The practitioner's guide. New York: John Wiley \& Sons.

Marsh, D. T. (2001). A Family-focused approach to serious mental illness: Empirically supported interventions. Sarasota, FL: Profssional Resources Press,

Marsh, D. T., Lefley, H. P., Ansell, V. I., Doerzbacher, B. M., Labarbera, L., et al. (1996). The family experience of mental illness: Evidence for resilience. Psychiatric Rehabilitation Journal, 20, 3-12.

McAdams, D. P., \& Olson, B. D. (2010). Personality development: Continuity and change over the life course. Annual Review of Psychology, 61, 517-542.

McClelland, D. C. (1951). Personality. New York: Dryden.

McCrae, R. R., \& Costa, P. T., Jr. (1991). Adding liebe und arbeit: The full fivefactor model and well-being. Personality and Social Psychology Bulletin, 17, 227-232.

McCubbin, H. I., \& McCubbin, M. A. (1988). Typologies of resilient families: Emerging roles of social class and ethnicity. Family Relations, 37, 247-254. Miller, J. B. (1976). Toward a new psychology of women. Boston: Beacon Press. 
Miller, G. E., \& Wrosch, C. (2007). You've gotta know when to fold 'em: Goal disengagement and systemic inflammation in adolescence. Psychological Science, 18, 773-777.

Miller, L. K., \& Hamblin, R. L. (1963). Interdependence, differential rewarding, and productivity. American Sociological Review, 28, 768-778.

Mishler, E. G., \& Waxler, N. E. (1965). Family interaction processes and schizophrenia: A review of curreny theories. Merrill-Palmer Quarterly, 11, 269-315.

Murphy, G. (1947). Personality: A biosocial approach to origins and structure. New York: Harper.

National Alliance for Caregiving (1997). Comparative analysis of caregiver data for caregivers to the elderly 1987 and 1997 . www.caregiving.org/data/analysis

Neugarten, B. L., Havighurst, R. J., \& Tobin, S. S. (1961). The measurement of life satisfaction. Journal of Gerontology, 16, 134-143.

Newcomb, M. D., \& Harlow, L. L. (1986). Life events and substance use among adolescents: Mediating effects of perceived loss of control and meaninglessness in life. Journal of Personality and Social Psychology, 51, 564-577.

Nolen-Hoeksema, S., Parker, L. E., \& Larson, J. (1994). Ruminative coping with depressed mood following loss. Journal of Personality and Social Psychology, 67, 92-104.

Noonan, A. E., \& Tennstedt, S. L. (1997). Meaning in caregiving and its contribution to caregiver well-being. Gerontologist, 37, 785-794. 
O'Connor, R. C., \& Forgan, G. (2007). Suicidal thinking and perfectionism: The role of goal adjustment amd behavioral inhibition/activation systems. Journal of Rational-Emotive \& Cognitive-Behavior Therapy, 25, 321-341.

Ory, M. G., Hoffman, R. R., 3rd, Yee, J. L., Tennstedt, S., \& Schulz, R. (1999). Prevalence and impact of caregiving: A detailed comparison between dementia and nondementia caregivers. Gerontologist, 39, 177-185.

Perneger, T. V. (1998), What's wrong with Bonferroni adjustments. Education and Debate, 316, 1234-1238.

Peterson, C. (2000). The future of optimism. American Psychologist, 55, 44-55.

Pickett, S. A., Cook, J. A., Cohler, B. J., \& Solomon, M. L. (1997). Positive parent/adult child relationships: Impact of severe mental illness and caregiving burden. American Journal of Orthopsychiatry, 67, 220-230.

Pinquart, M., \& Sorensen, S. (2003). Associations of stressors and uplifts of caregiving with caregiver burden and depressive mood: A meta-analysis. Journal of Gerontology Series B: Psychological Sciences and Social Sciences, $58,112-128$.

Pratt, C. C., Schmall, V. L., Wright, S., \& Cleland, M. (1985). Burden and coping strategies of caregivers to Alzheimer patients. Family Relations, 34, 27-33.

Radloff, L. S. (1977). The CES-D Scale: A self-report depression scale for research in the general population. Applied Psychological Measurement, 385-401.

Reinhard, S. C., \& Horwitz, A. V. (1995). Caregiver burden: Differentiating the content and consequences of family caregiving. Journal of Marriage and Family, 57, 741-750. 
Renshaw, K. D. (2008). The predictive, convergent, and discriminant validity of perceived criticism: A review. Clinical Psychology Review, 28, 521-534.

Rohde, P., Lewinsohn, P. M., Tilson, M., \& Seeley, J. R. (1990). Dimensionality of coping and its relation to depression. Journal of Personality and Social Psychology, 58, 499-511.

Rose, L. E. (1996). Families of psychiatric patients: A critical review and future research directions. Archive of Psychiatric Nursing, 10, 67-76.

Rosenheck, R., Cramer, J., Jurgis, G., Perlick, D., Xu, W., Thomas, J., et al. (2000). Clinical and psychopharmacologic factors influencing family burden in refractory schizophrenia. Journal of Clinical Psychiatry, 61, 671-676.

Roth, D. L., Perkins, M., Wadley, V. G., Temple, E. M., \& Haley, W. E. (2009). Family caregiving and emotional strain: Associations with quality of life in a large national sample of middle-aged and older adults. Quality of Life Research, 18, 679-688.

Ruggeri, M., Leese, M., Thornicroft, G., Bisoffi, G., \& Tansella, M. (2000). Definition and prevalence of severe and persistent mental illness. British Journal of Psychiatry, 177, 149-155.

Ryff, C. D. (1989a). Beyond Ponce de Leon and life satisfaction: New directions in quest for successful aging. International Journal of Behavioral Development, $12,35-55$.

Ryff, C. D. (1989b). Happiness is everything, or is it? Exploration on the meaning of psychological well-being. Journal of Personality and Social Psychology, $57,1069-1081$. 
Ryff, C. D. (1995). Psychological well-being in adult life. Current Directions in Psychological Science, 4, 99-104.

Ryff, C. D., Dienberg Love, G., Urry, H. L., Muller, D., Rosenkranz, M. A., Friedman, E. M., et al. (2006). Psychological well-being and ill-being: Do they have distinct or mirrored biological correlates? Psychotherapy and Psychosomatics, 75, 85-95.

Ryff, C. D., \& Keyes, C. L. (1995). The structure of psychological well-being revisited. Journal of Personality and Social Psychology, 69, 719-727.

Ryff, C. D., Lee, Y. H., \& Na, K. C. (1993). Through the lens of culture: Psychological well-being at midlife. Paper presented at the meeting of Gerontological Society of America, New Orleans, LA.

Scheier, M. F., \& Carver, C. S. (1985). Optimism, coping, and health: Assessment and implications of generalized outcome expectancies. Health Psychology, 4, 219-247.

Scheier, M. F., \& Carver, C. S. (1987). Dispositional optimism and physical wellbeing: The influence of generalized outcome expectancies on health. Journal of Personality, 55, 169-210.

Scheier, M. F., \& Carver, C. S. (1992). Effects of optimism on psychological and physical well-being: Theoretical overview and empirical update. Cognitive Therapy and Research, 16, 201-228.

Scheier, M. F., Carver, C. S., \& Bridges, M. W. (1994). Distinguishing optimism from neuroticism (and trait anxiety, self-mastery, and self-esteem): A 
reevaluation of the Life Orientation Test. Journal of Personality and Social Psychology, 67, 1063-1078.

Scheier, M. F., Carver, C. S., \& Bridges, M. W. (2001). Optimism, pessimism, and psychological well-being. In: E. C. Chang (Ed.), Optimism and pessimism (pp. 189-216). Washington DC: American Psychological Association.

Scheier, M. F., Matthews, K. A., Owens, J. F., Magovern, G. J., Sr., Lefebvre, R. C., Abbott, R. A., et al. (1989). Dispositional optimism and recovery from coronary artery bypass surgery: The beneficial effects on physical and psychological well-being. Journal of Personality and Social Psychology, 57, 1024-1040.

Scheier, M. F., Wrosch, C., Baum, A., Cohen, S., Martire, L. M., Matthews, K. A., et al. (2006). The life engagement test: Assessing purpose in life. Journal of Behavioral Medicine, 29, 291-298.

Schene, A. H., Tessler, R. C., \& Gamache, G. M. (1994). Instruments measuring family or caregiver burden in severe mental illness. Social Psychiatry and Psychiatric Epidemiology, 29, 228-240.

Schmutte, P. S., \& Ryff, C. D. (1997). Personality and well-being: Reexamining methods and meanings. Journal of Personality and Social Psychology, 73, 549-559.

Schulz, R. \& Beach, S. (1999). Caregiving as a risk factor for mortality: The caregiver health effects study. Journal of the American Medical Association, $282,2215-2219$. 
Schulz, R., Newsom, J., Mittelmark, M., Burton, L., Hirsch, C., \& Jackson, S. (1997). Health effects of caregiving: The caregiver health effects study: An ancillary study of the Cardiovascular Health Study. Annals of Behaioral Medicine, 19, 110-116.

Schulz, R., O'Brien, A. T., Bookwala, J., \& Fleissner, K. (1995). Psychiatric and physical morbidity effects of dementia caregiving: Prevalence, correlates, and causes. Gerontologist, 35, 771-791.

Seltzer, M. M., Greenberg, J. S., \& Krauss, M. W. (1995). A comparison of coping strategies of aging mothers of adults with mental illness or mental retardation. Psychology and Aging, 10, 64-75.

Singer, B. H., Ryff, C. D., Carr, D., \& Magee, W. J. (1998). Life histories and mental health: A person-centered strategy. In A. Raftery, (Ed.), Sociological Methodology (pp 1-51). Washington DC:American Sociological Association

Solomon, P., \& Draine, J. (1995). Adaptive coping among family members of persons with serious mental illness. Psychiatric Services, 46, 1156-1160.

Song, L. Y., Biegel, D. E., \& Milligan, S. E. (1997). Predictors of depressive symptomatology among lower social class caregivers of persons with chronic mental illness. Community Mental Health Journal, 33, 269-286.

Statistics Canada (2002) Balancing career and care. Perspectives. www.statcan.gc.ca/pub/75-001-x/11106/9520-eng.pdf

Stengard, E. (2002). Caregiving types and psychosocial well-being of caregivers of people with mental illness in Finland. Psychiatric Rehabilitation Journal, 26, 154-164. 
Swan, R. W., \& Lavitt, M. R. (1986). Patterns of adjustment to violence in families of the mentally ill. New Orleans, Elizabeth Wisner Research Center, Tulane University School of Social Work.

Talbott, J. A. (1983). The chronic mental patient: What have we learned? American Journal of Social Psychiatry, 3, 37-42.

Talley, R. C., \& Crews, J. E. (2007). Framing the public health of caregiving. American Journal of Public Health, 97, 224-228.

Taylor, S. E., \& Brown, J. D. (1988). Illusion and well-being: A social psychological perspective on mental health. Psychological Bulletin, 103, 193210.

Tellegen, A. (1985). Structures of moods and personality and their relevance to assessing anxiety, with an emphasis on self-report. In A. H.Tuma \& J. D. Maser (Eds.), Anxiety and anxiety disorders (pp. 681-716). Hillside, NJ: Erlbaum.

Tessler, R. C., \& Goldman, H. H. (1982). The chronically mentally ill: Assessing community support programs. Cambridge, MA: Ballinger.

Vaughn, C., \& Leff, J. (1976). The measurement of expressed emotion in the families of psychiatric patients. British Journal of Social and Clinical Psychology, 15, 157-165.

Wasow, M. (1995). The skipping stone: Ripple effects of mental illness on the family. Palo Alto, CA: Science \& Behavior Books.

Watson, D., \& Clark, L. A. (1984). Negative affectivity: The disposition to experience aversive emotional states. Psycholical Bulletin, 96, 465-490. 
Watson, D., Clark, L. A., \& Tellegen, A. (1988). Development and validation of brief measures of positive and negative affect: The PANAS scales. Journal of Personality and Social Psychology, 54, 1063-1070.

Wiggins, J. S. e. (1996). The five-factor model of personality: Theoretical perspectives. New York: Guilford.

Wrosch, C., Amir, E., \& Miller, G. E. (2011). Goal adjustment capacities, coping, and subjective well-being: The sample case of caregiving for a family member with mental illness. Journal of Personality and Social Psychology, 100, 934-946.

Wrosch, C., Bauer, I., \& Scheier, M. F. (2005). Regret and quality of life across the adult life span: The influence of disengagement and available future goals. Psychology and Aging, 20, 657-670.

Wrosch, C., Miller, G. E., Scheier, M. F., \& de Pontet, S. B. (2007). Giving up on unattainable goals: Benefits for health? Personality and Social Psychology Bulletin, 33, 251-265.

Wrosch, C., Miller, G. E., \& Schulz, R. (2009). Cortisol secretion and functional disabilities in old age: Importance of using adaptive control strategies. Psychosomatic Medicine, 71, 996-1003.

Wrosch, C., \& Scheier, M. F. (2003). Personality and quality of life: The importance of optimism and goal adjustment. Quality of Life Research, 12 (Suppl. 1), 59-72.

Wrosch, C., Scheier, M. F., Miller, G. E., Schulz, R., \& Carver, C. S. (2003). Adaptive self-regulation of unattainable goals: Goal disengagement, goal 
reengagement, and subjective well-being. Personality and Social Psychology Bulletin, 29, 1494-1508.

Yzerbyt, V. Y., Muller, D., \& Judd, C. M. (2004). Adjusting researchers' approach to adjustment: On the use of covariates when testing interactions. Journal of Experimental and Social Psychology, 40, 424-431.

Zarit, S. H., \& Zarit, J. M. (1987). The memory and behaviour problems checklist $1987 R$ and the burden interview (Technical report). State College, PA:

Pennsylvania State University. 


\section{amiquébec}

Agir contre la maladie mentale Action on mental illness

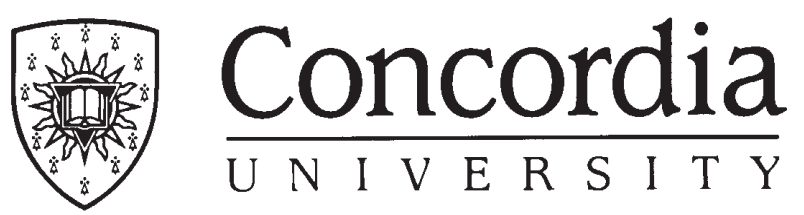

\section{Caregiver Study}

\#:

Date:

Dear Participant,

We want to thank you for participating in this study. Without your answers, we could not do research. Please keep in mind that all the information you provide is absolutely confidential and will only be used for research purposes. If you have any questions or need further clarification, please do not hesitate to contact us.

\section{Ella Amir}

Researcher/Executive Director AMI Quebec

Joelle Jobin

Research Coordinator

Tel: (514) 848-2424 Ext. 2236

Email: cwlab@alcor.concordia.ca

\section{Dr. Carsten Wrosch Principal Researcher Concordia University}




\section{PERSONAL INFORMATION}

Please check off the appropriate box.

$\operatorname{Sex} \square$ Female $\quad \square$ Male

Age $\longrightarrow$ yrs

First Language $\square$ English $\quad \square$ French $\quad \square$ Other

Family Status?

Single

Live with a partner but not married

Married

$\square$ Divorced; please indicate since when

$\square$ Widowed; please indicate since when

Highest Level of Education Completed

$\square$ None
$\square$ Grade School
$\square$ High School
$\square$ Collegial or Trade School
$\square$ Bachelor's Degree
$\square$ Master's or Doctorate Degree

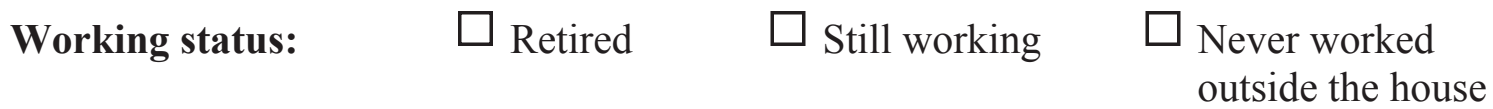

Profession

Current Family income (per year):

Less than $17000 \$$

$51001 \$-68000 \$$
$17001 \$-34000 \$$

$68001 \$-85000 \$$
$34001 \$-51000 \$$

$\square$ more than 85000 \$ 


\section{DESCRIBE YOURSELF}

1. Using the scale below, place a check beside each statement that indicates the extent to which you agree or disagree. Please be as accurate and honest as possible, and remember there are no right or wrong answers.

\begin{tabular}{|l|l|l|l|l|l||}
\hline & $\begin{array}{c}\text { Strongly } \\
\text { Disagree }\end{array}$ & Disagree & Neutral & Agree & $\begin{array}{c}\text { Strongly } \\
\text { Agree }\end{array}$ \\
\hline 1. In uncertain times, I usually expect the best. & & & & & \\
\hline 2. There is not enough purpose in my life. & & & & & \\
\hline 3. It's easy for me to relax. & & & & & \\
\hline 4. To me, the things I do are all worthwhile. & & & & & \\
\hline 5. If something can go wrong for me it will. & & & & & \\
\hline 6. Most of what I do seems trivial and \\
unimportant to me.
\end{tabular}




\section{Satisfaction with life}

\section{DESCRIBE YOURSELF}

2. Please indicate your level of agreement with each of the following five statements by checking under the appropriate column.

\begin{tabular}{||l|l|l|l|l|l||}
\hline & $\begin{array}{c}\text { Strongly } \\
\text { Disagree }\end{array}$ & Disagree & Neutral & Agree & $\begin{array}{c}\text { Strongly } \\
\text { Agree }\end{array}$ \\
\hline $\begin{array}{l}\text { 1. In most ways my life is close to } \\
\text { my ideal. }\end{array}$ & & & & & \\
\hline $\begin{array}{l}\text { 2. The conditions of my life are } \\
\text { excellent. }\end{array}$ & & & & & \\
\hline 3. I am satisfied with my life. & & & & & \\
\hline $\begin{array}{l}\text { 4. So far I have gotten important } \\
\text { things I want in life. }\end{array}$ & & & & & \\
\hline $\begin{array}{l}\text { 5. If I could live my life over, I } \\
\text { would change almost everything. }\end{array}$ & & & & & \\
\hline \hline
\end{tabular}




\section{Unmitigated communion}

\section{DESCRIBE YOURSELF}

3. Using the scale below, place a check beside each statement that indicates the extent to which you agree or disagree. Think of people close to you (i.e., family members) in responding to each statement.

\begin{tabular}{||c|l|l|l|l|l|}
\hline & $\begin{array}{c}\text { Strongly } \\
\text { Disagree }\end{array}$ & $\begin{array}{c}\text { Slightly } \\
\text { Disagree }\end{array}$ & $\begin{array}{c}\text { Neither } \\
\text { Agree nor } \\
\text { Disagree }\end{array}$ & $\begin{array}{c}\text { Slightly } \\
\text { Agree }\end{array}$ & $\begin{array}{c}\text { Strongly } \\
\text { Agree }\end{array}$ \\
\hline $\begin{array}{c}\text { 1. I always place the needs of others above } \\
\text { my own. }\end{array}$ & & & & & \\
\hline $\begin{array}{c}\text { 2. I never find myself getting overly } \\
\text { involved in others' problems. }\end{array}$ & & & & & \\
\hline $\begin{array}{c}\text { 3. For me to be happy, I need others to be } \\
\text { happy. }\end{array}$ & & & & & \\
\hline $\begin{array}{l}\text { 4. I worry about how other people get along } \\
\text { without me when I am not there. }\end{array}$ & & & & & \\
\hline $\begin{array}{l}\text { 5. I have no trouble getting to sleep at night } \\
\text { when other people are upset. }\end{array}$ & & & & & \\
\hline $\begin{array}{c}\text { 6. It is impossible for me to satisfy my own } \\
\text { needs when they interfere with the needs } \\
\text { of others. }\end{array}$ & & & & & \\
\hline $\begin{array}{l}\text { 7. I can't say no when someone asks me for } \\
\text { help. }\end{array}$ & & & & & \\
\hline $\begin{array}{l}\text { 8. Even when exhausted, I will always help } \\
\text { other people. }\end{array}$ & & & & & \\
\hline \begin{tabular}{l} 
9. I often worry about others' problems. \\
\hline
\end{tabular} & & & & & \\
\hline
\end{tabular}




\section{DESCRIBE YOURSELF}

4. This scale consists of a number of words that describe different feelings and emotions. Read each item and indicate to what extent you experienced the following emotions during the past year.

\begin{tabular}{|c|c|c|c|c|c|}
\hline & $\begin{array}{l}\text { Very slightly } \\
\text { or not at all }\end{array}$ & A little & Moderately & Quite a bit & Extremely \\
\hline \multicolumn{6}{|c|}{ 1. Interested } \\
\hline \multicolumn{6}{|c|}{ 2. Distressed } \\
\hline \multicolumn{6}{|l|}{ 3. Excited } \\
\hline \multicolumn{6}{|l|}{ 4. Upset } \\
\hline \multicolumn{6}{|l|}{ 5. Strong } \\
\hline \multicolumn{6}{|l|}{ 6. Guilty } \\
\hline \multicolumn{6}{|l|}{ 7. Scared } \\
\hline \multicolumn{6}{|l|}{ 8. Hostile } \\
\hline \multicolumn{6}{|c|}{ 9. Enthusiastic } \\
\hline \multicolumn{6}{|l|}{ 10. Proud } \\
\hline \multicolumn{6}{|l|}{ 11. Irritable } \\
\hline \multicolumn{6}{|l|}{ 12. Alert } \\
\hline \multicolumn{6}{|c|}{ 13. Ashamed } \\
\hline \multicolumn{6}{|l|}{ 14. Inspired } \\
\hline \multicolumn{6}{|c|}{ 15. Nervous } \\
\hline \multicolumn{6}{|c|}{ 16. Determined } \\
\hline \multicolumn{6}{|c|}{ 17. Attentive } \\
\hline \multicolumn{6}{|l|}{ 18. Jittery } \\
\hline \multicolumn{6}{|l|}{ 19. Active } \\
\hline 20. Afraid & & & & & \\
\hline
\end{tabular}




\section{DESCRIBE YOURSELF}

5. Below is a list of the ways you might have felt or behaved. Please indicate how often you have felt this way during the past week by using the following scale:

$$
\begin{aligned}
& 1 \text { = Rarely or None of the time (Less than } 1 \text { Day) } \\
& 2 \text { = Some or a Little of the Time (1 - } 2 \text { Days) } \\
& 3 \text { = Occasionally or a Moderate Amount of Time (3 - } 4 \text { Days) } \\
& 4 \text { = Most or All of the Time ( } 5 \text { - } 7 \text { Days) }
\end{aligned}
$$

During the past week:

1. I was bothered by things that usually don't bother me.

2. I I had trouble keeping my mind on what I was doing.

3. I felt depressed.

4. I f felt that everything I did was an effort.

5. I I felt hopeful about the future.

6._ I felt fearful.

7.__ My sleep was restless.

8. I I was happy.

9._ I felt lonely.

10. _ _ I could not get "going."

11.__ I did not feel like eating; appetite was poor.

12. I I felt I could not shake off the blues, even with help from family and friends.

13. I I felt that I was just as good as other people

14. I I thought my life had been a failure.

15. I I talked less than usual.

16. People were unfriendly.

17. I enjoyed life.

18. I L I had crying spells.

19._ I felt sad.

20. I f f _ I that people disliked me. 


\section{G. Goal adjustment capacities}

\section{UNATTAINABLE GOALS}

During their lives people cannot always attain what they want and are sometimes forced to stop pursuing the goals they have set. We are interested in understanding how you usually react when this happens to you. Please indicate the extent to which you agree or disagree with each of the following statements, as it usually applies to you.

If I have to stop pursuing an important goal in my life...

\begin{tabular}{|c|c|c|c|c|c|}
\hline & $\begin{array}{l}\text { Strongly } \\
\text { Disagree }\end{array}$ & Disagree & Neutral & Agree & $\begin{array}{c}\text { Strongly } \\
\text { Agree }\end{array}$ \\
\hline $\begin{array}{l}\text { 1. It's easy for me to reduce my effort } \\
\text { towards the goal. }\end{array}$ & & & & & \\
\hline $\begin{array}{l}\text { 2. I convince myself that I have other } \\
\text { meaningful goals to pursue. }\end{array}$ & & & & & \\
\hline $\begin{array}{l}\text { 3. I stay committed to the goal for a long } \\
\text { time; I can't let it go. }\end{array}$ & & & & & \\
\hline 4. I start working on other new goals. & & & & & \\
\hline 5. I think about other new goals to pursue & & & & & \\
\hline $\begin{array}{l}\text { 6. I find it difficult to stop trying to achieve } \\
\text { the goal. }\end{array}$ & & & & & \\
\hline 7. I seek other meaningful goals. & & & & & \\
\hline $\begin{array}{l}\text { 8. It's easy for me to stop thinking about the } \\
\text { goal and let it go. }\end{array}$ & & & & & \\
\hline $\begin{array}{l}\text { 9. I tell myself that I have a number of other } \\
\text { new goals to draw upon. }\end{array}$ & & & & & \\
\hline $\begin{array}{l}\text { 10. I put effort toward other meaningful } \\
\text { goals. }\end{array}$ & & & & & \\
\hline
\end{tabular}




\section{H. Coping with stress}

\section{COPING WITH STRESSFUL SITUATIONS}

These items deal with ways you've been coping with the stress in your life since you found out your relative had a mental illness. There are many ways to try to deal with problems. These items ask what you've been doing to cope with this one. Obviously, different people deal with things in different ways, but I'm interested in how you've tried to deal with it. I want to know to what extent you've been doing what the items says. How much or how frequently. Don't answer on the basis of whether it seems to be working or not - just whether or not you're doing it. Use these response choices. Try to rate each item separately in you mind from the others. Make your answers as true FOR YOU as you can.

\begin{tabular}{|c|c|c|c|c|}
\hline & $\begin{array}{l}\text { I haven't } \\
\text { been } \\
\text { doing this } \\
\text { at all }\end{array}$ & $\begin{array}{c}\text { I've been } \\
\text { doing this a } \\
\text { little bit }\end{array}$ & \begin{tabular}{|c|} 
I've been \\
doing this a \\
medium \\
amount \\
\end{tabular} & $\begin{array}{c}\text { I've been } \\
\text { doing this } \\
\text { a lot }\end{array}$ \\
\hline \multicolumn{5}{|l|}{$\begin{array}{l}\text { 1. I concentrate my efforts on doing something } \\
\text { about the situations I find myself in. }\end{array}$} \\
\hline \multicolumn{5}{|l|}{$\begin{array}{l}\text { 2. I try to see it in a different light, to make it } \\
\text { seem more positive. }\end{array}$} \\
\hline \multicolumn{5}{|l|}{ 3. I make jokes about it. } \\
\hline \multicolumn{5}{|l|}{ 4. I get emotional support from others. } \\
\hline \multicolumn{5}{|l|}{$\begin{array}{l}\text { 5. I turn to work or other activities to take my } \\
\text { mind off things. }\end{array}$} \\
\hline \multicolumn{5}{|l|}{ 6. I say to myself "this isn't real". } \\
\hline \multicolumn{5}{|l|}{ 7. I express my negative feelings. } \\
\hline \multicolumn{5}{|l|}{ 8. I give up the attempt to cope. } \\
\hline \multicolumn{5}{|c|}{$\begin{array}{l}\text { 9. I try to come up with a strategy about what to } \\
\text { do. }\end{array}$} \\
\hline \multicolumn{5}{|l|}{ 10. I learn to live with it. } \\
\hline \multicolumn{5}{|l|}{$\begin{array}{l}\text { 11. I find comfort in my religion or spiritual } \\
\text { beliefs. }\end{array}$} \\
\hline \multicolumn{5}{|l|}{$\begin{array}{l}\text { 12. I use alcohol or other drugs to make myself } \\
\text { feel better. }\end{array}$} \\
\hline \multicolumn{5}{|l|}{ 13. I criticize myself. } \\
\hline \multicolumn{5}{|l|}{ 14. I get help and advice from other people. } \\
\hline \multicolumn{5}{|l|}{ 15. I give up trying to deal with it. } \\
\hline $\begin{array}{l}\text { 16. I say things to let my unpleasant feelings } \\
\text { escape. }\end{array}$ & & & & \\
\hline
\end{tabular}




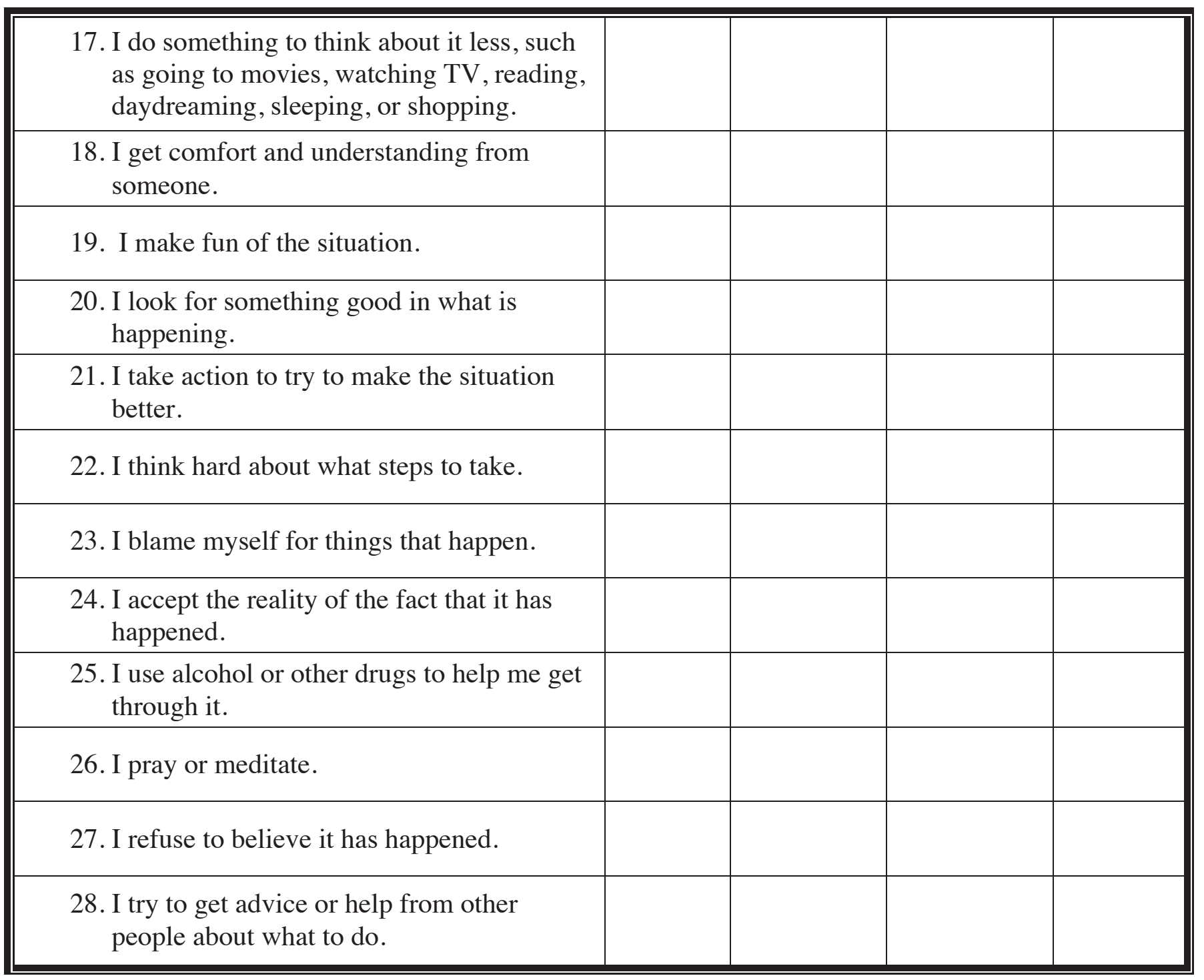




\section{HEALTH}

\section{Please answer the following questions about you physical health.}

\begin{tabular}{|c|c|c|c|}
\hline & NO & YES & $\begin{array}{l}\text { NOT } \\
\text { SURE }\end{array}$ \\
\hline 1. Do you currently have high blood pressure? & & & \\
\hline $\begin{array}{l}\text { 2. Do you currently have problems with an irregular heartbeat } \\
\text { or chest pain? }\end{array}$ & & & \\
\hline $\begin{array}{l}\text { 3. Have you ever been told that you have coronary heart } \\
\text { disease or coronary artery disease? }\end{array}$ & & & \\
\hline 4. Have you ever had a heart attack? & & & \\
\hline 5. Have you ever been treated for congestive heart failure? & & & \\
\hline $\begin{array}{l}\text { 6. Have you ever had major surgery? } \\
\text { (IF YES) What? }\end{array}$ & & & \\
\hline 7. Have you ever had a stroke? & & & \\
\hline $\begin{array}{l}\text { 8. Do you currently have osteoarthritis, fibromyalgia, } \\
\text { osteoporosis, or any other serious muscular or bone } \\
\text { problem? }\end{array}$ & & & \\
\hline $\begin{array}{l}\text { 9. Do you currently have asthma, emphysema, chronic } \\
\text { bronchitis, chronic obstructive lung disease, or any other } \\
\text { serious respiratory problems? }\end{array}$ & & & \\
\hline $\begin{array}{l}\text { 10. Do you currently have stomach ulcers, irritable bowel } \\
\text { syndrome, or any other serious problems with you stomach } \\
\text { or bowels? }\end{array}$ & & & \\
\hline 11. Do you have diabetes? & & & \\
\hline 12. Do you currently have problems with your kidneys? & & & \\
\hline 13. Do you have cirrhosis or any other serious liver problems? & & & \\
\hline $\begin{array}{l}\text { 14. Do you currently have cancer? } \\
\text { (IF YES) What? }\end{array}$ & & & \\
\hline $\begin{array}{l}\text { 15. Do you currently have rheumatoid arthritis, lupus, acquired } \\
\text { immune deficiency syndrome, multiple sclerosis, } \\
\text { scleroderma, or any other autoimmune problem? }\end{array}$ & & & \\
\hline $\begin{array}{l}\text { 16. Do you currently have problems with blood circulation in } \\
\text { your legs, hemophilia, or any other blood-related } \\
\text { problems? }\end{array}$ & & & \\
\hline
\end{tabular}




\begin{tabular}{||l|l|l|l|}
\hline & NO & YES & $\begin{array}{c}\text { NOT } \\
\text { SURE }\end{array}$ \\
\hline $\begin{array}{l}\text { 17. Do you have epilepsy or any other neurological problems? } \\
\text { 18. Do you currently have an overactive or underactive } \\
\text { thyroid, or any other thyroid problems? }\end{array}$ & & & \\
\hline $\begin{array}{l}\text { 19. Do you currently have any problems with you vision or } \\
\text { hearing? }\end{array}$ & & & \\
\hline $\begin{array}{l}\text { 20. Do you currently have asthma, bronchitis, or emphysema? } \\
\text { 21. Do you currently have persistent skin trouble (e.g., } \\
\text { eczema)? }\end{array}$ & & & \\
\hline $\begin{array}{l}\text { 22. Do you currently have recurring stomach trouble, } \\
\text { indigestion, or diarrhea? }\end{array}$ & & & \\
\hline 23. Do you currently have migrane headaches? & & & \\
\hline 24. Are you constipated all or most of the time? & & & \\
\hline 25. Do you have chronic sleeping problems? & & \\
\hline $\begin{array}{l}\text { 26. Do you currently have any other health problems that I } \\
\text { have not asked you about? }\end{array}$ & & & \\
\hline \begin{tabular}{l} 
(IF YES) What? \\
\hline
\end{tabular}
\end{tabular}

\section{This week, have you been bothered by}

\begin{tabular}{||l|l|l||}
\hline & NO & YES \\
\hline 1. Stomach pain & & \\
\hline 2. Back pain & & \\
\hline 3. Pain in your arms, legs or joints (knees hips, etc.) & & \\
\hline 5. $\quad$ Headaches & & \\
\hline 6. Chest pain & & \\
\hline 7. Dizziness & & \\
\hline 8. Fainting Spells & & \\
\hline 9. Feeling your heart pound or race & & \\
\hline 10. Shortness of breath & & \\
\hline 11. Constipation, loose bowels, or diarrhea & & \\
\hline 12. Nausea, gas or indigestion & & \\
\hline \hline
\end{tabular}




\section{RELATIVE CHARACTERISTICS}

Please check off the appropriate box that describes the relative that you care for.

The relative I care for is:
My spouse
My child
My sibling
My parent
Other, please specify:

My relative is: $\square$ Male $\square$ Female

Age of the relative:

Age of relative at first hospitalization:

Number of times relative has been hospitalized:

My relative was diagnosed with a mental illness ___ years ago.

What diagnosis did your relative receive?

Where does the relative you care for live?
With you
Independently
Assisted Living Facility
Other, please specify:

If you were to rate your relative's illness on a scale 1-5, with 5 being the most severe form of illness, how would you rate them?

1-------------------------------2----------------------------3------------------------4--------------------------5 


\section{K. Burden}

The following questions ask about how often certain situations arise as a result of any emotional support of physical assistance that you provide to your relative. Please answer the following questions:

\section{How often do you feel...}

\begin{tabular}{|c|c|c|c|c|c|}
\hline & Never & Rarely & Sometimes & $\begin{array}{c}\text { Quite } \\
\text { Frequently }\end{array}$ & $\begin{array}{l}\text { Nearly } \\
\text { Always }\end{array}$ \\
\hline $\begin{array}{l}\text { 1. That your relative asks for more } \\
\text { help then they need? }\end{array}$ & & & & & \\
\hline $\begin{array}{l}\text { 2. That because of the time you spen } \\
\text { with your relative you don't have } \\
\text { enough time for yourself? }\end{array}$ & & & & & \\
\hline $\begin{array}{l}\text { 3. Stressed between caring for your } \\
\text { relative and trying to meet other } \\
\text { responsibilities for your family or } \\
\text { work? }\end{array}$ & & & & & \\
\hline $\begin{array}{l}\text { 4. Embarrassed over your relative's } \\
\text { behavior? }\end{array}$ & & & & & \\
\hline $\begin{array}{l}\text { 5. Angry when you are around your } \\
\text { relative? }\end{array}$ & & & & & \\
\hline
\end{tabular}

\begin{tabular}{|c|c|c|c|c|c|}
\hline & Never & Rarely & Sometimes & $\begin{array}{c}\text { Quite } \\
\text { Frequently }\end{array}$ & $\begin{array}{l}\text { Nearly } \\
\text { Always }\end{array}$ \\
\hline $\begin{array}{l}\text { 6. That your relative currently affects } \\
\text { your relationships with other family } \\
\text { members or friends in a negative way? }\end{array}$ & & & & & \\
\hline $\begin{array}{l}\text { 7. Afraid of what the future holds for } \\
\text { your relative? }\end{array}$ & & & & & \\
\hline $\begin{array}{l}\text { 8. That your relative is dependent on } \\
\text { you? }\end{array}$ & & & & & \\
\hline $\begin{array}{l}\text { 9. Strained when you are around your } \\
\text { relative }\end{array}$ & & & & & \\
\hline $\begin{array}{l}\text { 10. Your health has suffered because of } \\
\text { your involvement with your relative? }\end{array}$ & & & & & \\
\hline $\begin{array}{l}\text { 11. That you don't have as much privacy } \\
\text { as you would like because of your } \\
\text { relative? }\end{array}$ & & & & & \\
\hline $\begin{array}{l}\text { 12. That your social life has suffered } \\
\text { because you are caring for your } \\
\text { relative? }\end{array}$ & & & & & \\
\hline
\end{tabular}




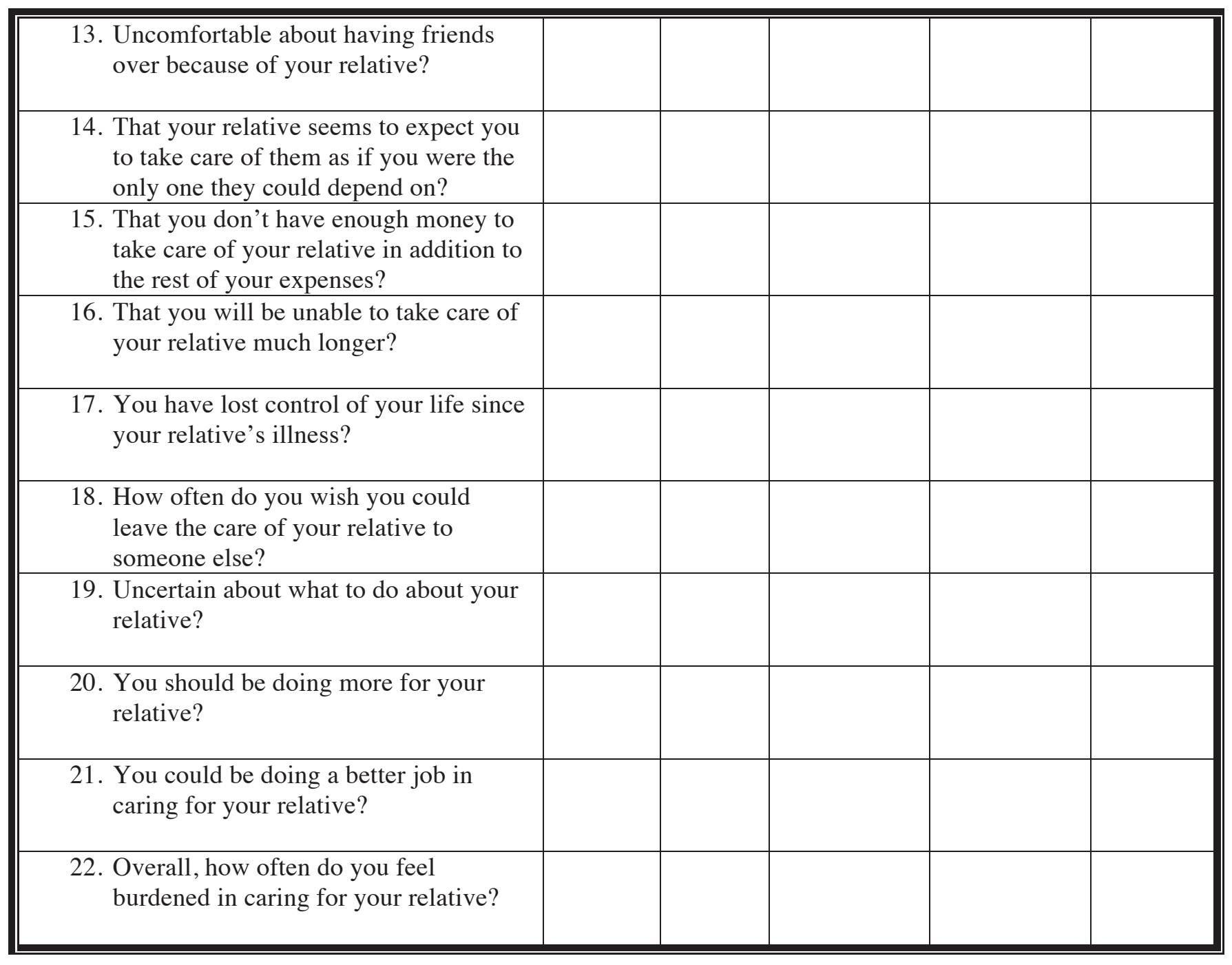




\section{CONSENT TO PARTICIPATE IN “PATHWAYS TO WELL-BEING AND HEALTH AMONG FAMILIES LIVING WITH MENTAL ILLNESS"}

This is to state that I agree to participate in a program of research being conducted by Dr. Carsten Wrosch of the Psychology Department of Concordia University (phone: 514 848-2424 ext. 2231; email: Carsten.wrosch@concordia.ca). This research project is conducted in collaboration with AMI Québec (Ella Amir, Executive Director; phone: 514 486-1448; email: ellaamir@amiquebec.org). Ella Amir is also a Ph. D. candidate at Concordia University.

\section{A. PURPOSE}

I have been informed that the purpose of the research is to examine pathways to subjective well-being and physical health among families who have a relative with mental illness.

\section{B. PROCEDURES}

The research involves responding to two questionnaires over a period of approximately two years. You have already responded to the first questionnaire and are now being sent the second questionnaire by mail. Responding to the questionnaire will take approximately one hour of your time. The questionnaire should be returned to Dr. Wrosch's laboratory once completed.

\section{RISKS AND BENEFITS}

There should be no risk of participating in this research. The questions ask only about your opinion about yourself, your situation, and circumstances. If you feel discomfort at any time during your participation in this study, please contact either Dr. Wrosch or Ella Amir at AMI Québec. Your participation may help us to illuminate factors that contribute to well-being and health among families who have a relative with mental illness.

\section{CONDITIONS OF PARTICIPATION}

- I understand that I am free to withdraw my consent and discontinue my participation at anytime without negative consequences.

- I understand that my participation in this study is CONFIDENTIAL (i.e., the researcher will know, but will not disclose my identity)

- I understand that the data from this study may be published.

I HAVE CAREFULLY STUDIED THE ABOVE AND UNDERSTAND THIS AGREEMENT. I FREELY CONSENT AND VOLUNTARILY AGREE TO PARTICIPATE IN THIS STUDY.

NAME (please print) 


\section{SIGNATURE}

If at any time you have questions about your rights as a research participant, please contact Dr. Brigitte Des Rosiers, Research Ethics and Compliance Officer, Concordia University, at (514) 848-2424 x 7481 or by email at bdesrosi@alcor.concordia.ca. 
November, 2007

Dear AMI member:

Having a mental illness or caring for a relative with mental illness is a demanding, longterm preoccupation, which often takes a high toll on the family.

The better we understand some of the factors that lead to positive outcomes among people with mental illness or care about a person with mental illness, the better we can design programs that could, ultimately, alleviate some of the burden associated with it.

I have been working with families for the past 17 years, and being able to help is dear to my heart. For that reason I have embarked on a research project in which I hope you would agree to participate. This project is part of a PhD program in which I am presently enrolled, under the supervision of Dr. Carsten Wrosch from the Psychology Department of Concordia University.

We would like to ask you to participate in this research. It includes two questionnaires, to which you will be asked to respond over the course of two years. Completing each questionnaire is expected to take less than one hour. Your participation will enable us to better help families who are dealing with mental illness, like yourself, and we would be very grateful if you accept our invitation.

We will contact you by phone over the coming weeks. However, if you are NOT interested in participating in our study, please send us the enclosed response card and we will not contact you. Please keep in mind that all your answers will be kept strictly confidential, and the results of this project will be used only for research purposes and transferring research knowledge into programs to help families.

Thank you very much for your time!

Sincerely,

Ella Amir

Executive Director

AMI-Québec

Tel: (514) 486-1448
Carsten Wrosch, Ph.D.

Aging, Personality, and Health

Research Laboratory

Concordia University

Tel: (514) 848-2424 Ext. 2236 


\section{amiquébec}

Agir contre la maladie mentale

Action on mental illness

Dear Participant,

October 2009

In Spring 2008 you participated in the first phase of a study on the experience of caregiving to a person with mental illness. This study was conducted at Concordia University, in collaboration with AMI-Quebec, and more than 150 individuals participated. We wish to thank you once again for helping us better understand some of the factors that lead to positive outcomes for family members who care for a person with mental illness. This understanding is important for the design of programs that could alleviate some of the burdens.

Now we are embarking on the second phase of the study that includes another questionnaire. While some of the questions are very similar to the questions you have answered last year, completing the second questionnaire would be particularly critical for the success of the project. To identify the factors that are causally involved in positive outcomes among caregivers, we need to examine whether the experience of caregiving has been stable or has changed over time. Without this information, the results of the study would remain preliminary and we would not be able to illuminate pathways to well-being and health among caregivers Your participation in the second phase, therefore, is very important to us, and we sincerely hope that you will help us one more time.

You will find enclosed a consent form and a questionnaire. Please read and sign the consent form before answering the questionnaire. The consent form explains your rights as a participant. By signing this document you authorize us to use your answers to the questionnaire for research purposes only. We must emphasize that all of the information you provide will remain strictly confidential. Should these findings be published, there will be no mention of the individuals who participated in the study. The only place where we require your name is on the consent form and this will be kept separate from the questionnaire so no association can be made between your name and your responses.

Every effort has been made to ensure that the questions are as clear and non-intrusive as possible. However, if for any reason you do not understand a question or are unwilling to provide a response, feel free to leave that question blank or contact us for further clarification. We can be reached by phone at 514-848-2424 (extension 2236) or by email at:

cwlab@alcor.concordia.ca. Please take the time to read the instructions and questions carefully but do not dwell too long on any one answer. We are interested in your initial impressions. There are no right or wrong answers. Please be honest and candid.

Once completed, please send the questionnaires and the consent form back to us in the selfaddressed and pre-paid envelope. We would appreciate your response within the next month, and wish to thank you for your interest and participation.

Sincerely,

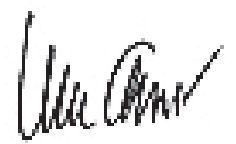

Ella Amir

Executive Director, AMI-Quebec

Tel: (514) 486-1448

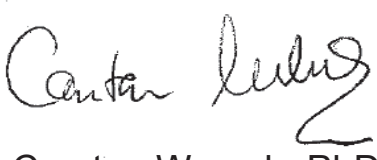

Carsten Wrosch, PhD

Associate Professor, Concordia University

Tel: (514) 848-2424 Ext. 2236 